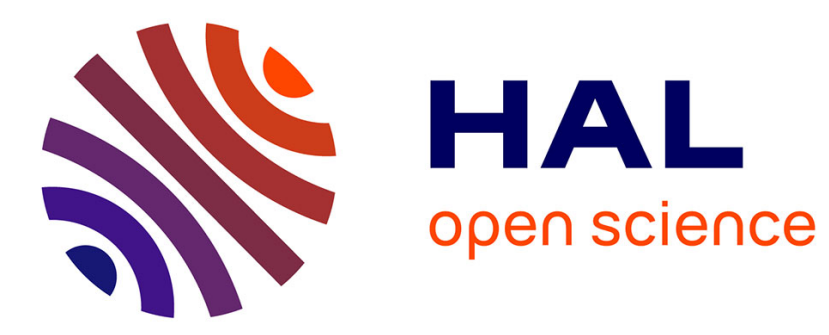

\title{
Physical, spectral, and dynamical properties of asteroid (107) Camilla and its satellites
}

M. Pajuelo, B. Carry, F. Vachier, M. Marsset, J. Berthier, P. Descamps, J. Merline, M. Tamblyn, J. Grice, A. Conrad, et al.

\section{- To cite this version:}

M. Pajuelo, B. Carry, F. Vachier, M. Marsset, J. Berthier, et al.. Physical, spectral, and dynamical properties of asteroid (107) Camilla and its satellites. Icarus, 2018, 309, pp.134-161. 10.1016/j.icarus.2018.03.003 . hal-01946888

\section{HAL Id: hal-01946888 \\ https://hal.sorbonne-universite.fr/hal-01946888}

Submitted on 6 Dec 2018

HAL is a multi-disciplinary open access archive for the deposit and dissemination of scientific research documents, whether they are published or not. The documents may come from teaching and research institutions in France or abroad, or from public or private research centers.
L'archive ouverte pluridisciplinaire HAL, est destinée au dépôt et à la diffusion de documents scientifiques de niveau recherche, publiés ou non, émanant des établissements d'enseignement et de recherche français ou étrangers, des laboratoires publics ou privés. 


\title{
Physical, spectral, and dynamical properties of asteroid (107) Camilla and its satellites
}

\author{
M. Pajuelo ${ }^{\mathrm{a}, \mathrm{b}, *}$, B. Carry ${ }^{\mathrm{a}, \mathrm{c}}$, F. Vachier ${ }^{\mathrm{a}}$, M. Marsset ${ }^{\mathrm{d}}$, J. Berthier ${ }^{\mathrm{a}}$, P. Descamps ${ }^{\mathrm{a}}$,

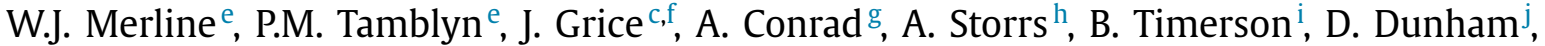 \\ S. Preston ${ }^{\mathrm{k}}$, A. Vigan ${ }^{1}$, B. Yang ${ }^{\mathrm{m}}$, P. Vernazza ${ }^{1}$, S. Fauvaud ${ }^{\mathrm{n}}$, L. Bernasconi ${ }^{\circ}$, D. Romeuf ${ }^{\circ}$, \\ R. Behrend ${ }^{\mathrm{o}, \mathrm{r}}$, C. Dumas ${ }^{\mathrm{m}, \mathrm{p}}$, J.D. Drummond ${ }^{\mathrm{q}}$, J.-L. Margot ${ }^{\mathrm{s}}$, P. Kervella ${ }^{\mathrm{t}, \mathrm{u}}$, F. Marchis ${ }^{\mathrm{v}}$, \\ J.H. Girard ${ }^{w}$
}

a IMCCE, Observatoire de Paris, PSL Research University, CNRS, Sorbonne Universités, UPMC Univ Paris 06, Univ. Lille, France

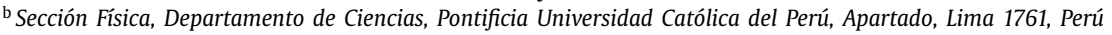

' Université Côte d'Azur, Observatoire de la Côte d'Azur, CNRS, Laboratoire Lagrange, France

${ }^{\mathrm{d}}$ Astrophysics Research Centre, Queen's University Belfast, Belfast, County Antrim, BT7 1NN, UK

e Southwest Research Institute, Boulder, CO, USA

${ }^{\mathrm{f}}$ School of Physical Sciences, The Open University, MK7 6AA, UK

${ }^{g}$ Large Binocular Telescope Observatory, University of Arizona, Tucson, AZ 85721, USA

${ }^{\mathrm{h}}$ Towson University, Towson, MD, USA

i International Occultation Timing Association (IOTA), 623 Bell Rd., Newark, NY 14513-8805, USA

j IOTA, 3719 Kara Ct., Greenbelt, MD 20770-3016, USA

k IOTA, 7640 NE 32 nd St., Medina, WA 98039, USA

${ }^{1}$ Aix Marseille Univ, CNRS, LAM, Laboratoire d'Astrophysique de Marseille, Marseille, France

m ESO-Chile, Alonso de Córdova 3107, Vitacura, Santiago, RM, Chile

n Observatoire du Bois de Bardon, 16110, Taponnat, France

${ }^{\circ}$ CdR \& CdL Group: Lightcurves of Minor Planets and Variable Stars, Switzerland

${ }^{\mathrm{p}}$ Thirty-Meter-Telescope, 100 West Walnut St, Suite 300, Pasadena, CA 91124, USA

${ }^{q}$ Leidos, Starfire Optical Range, AFRL/RDS, Kirtland AFB, NM 87117, USA

${ }^{\mathrm{r}}$ Geneva Observatory, Sauverny 1290, Switzerland

${ }^{\mathrm{s}}$ Department of Earth, Planetary, and Space Sciences, UCLA, Los Angeles, CA 90095, USA

${ }^{t}$ Unidad Mixta Internacional Franco-Chilena de Astronomía, CNRS/INSU UMI 3386 and Departamento de Astronomía, Universidad de Chile, Casilla 36-D, Santiago, Chile

"LESIA, Observatoire de Paris, PSL Research University, CNRS, Sorbonne Universités, UPMC Univ. Paris 06, Univ. Paris Diderot, Sorbonne Paris Cité, 5 Place Jules Janssen, Meudon 92195, France

v SETI Institute, Carl Sagan Center, 189 Bernado Avenue, Mountain View, CA 94043, USA

w Space Telescope Science Institute, 3700 San Martin Drive, Baltimore, MD 21218, USA

Keywords:

Asteroids

Composition

Satellites of asteroids

Photometry

Spectroscopy

\section{A B S T R A C T}

The population of large $100+\mathrm{km}$ asteroids is thought to be primordial. As such, they are the most direct witnesses of the early history of our Solar System available. Those among them with satellites allow study of the mass, and hence density and internal structure. We study here the dynamical, physical, and spectral properties of the triple asteroid (107) Camilla from lightcurves, stellar occultations, optical spectroscopy, and high-contrast and high-angular-resolution images and spectro-images.

Using 80 positions measured over 15 years, we determine the orbit of its larger satellite, $S / 2001$ (107) 1 , to be circular, equatorial, and prograde, with root-mean-square residuals of 7.8 mas, corresponding to a sub-pixel accuracy. From 11 positions spread over three epochs only, in 2015 and 2016, we determine a preliminary orbit for the second satellite $S / 2016$ (107) 1 . We find the orbit to be somewhat eccentric and slightly inclined to the primary's equatorial plane, reminiscent of the properties of inner satellites of other asteroid triple systems. Comparison of the near-infrared spectrum of the larger satellite reveals no significant difference with Camilla. Hence, both dynamical and surface properties argue for a formation of the satellites by excavation from impact and re-accumulation of ejecta in orbit.

We determine the spin and 3-D shape of Camilla. The model fits well each data set: lightcurves, adaptiveoptics images, and stellar occultations. We determine Camilla to be larger than reported from modeling of mid-infrared photometry, with a spherical-volume-equivalent diameter of $254 \pm 36 \mathrm{~km}$ ( $3 \sigma$ uncertainty), in agreement with recent results from shape modeling (Hanus et al., 2017, A\&A 601). Combining the mass of $(1.12 \pm 0.01) \times 10^{19} \mathrm{~kg}$ ( $3 \sigma$ uncertainty) determined from the dynamics of the satellites and the volume from the 3-D shape model, we determine a density of $1,280 \pm 130 \mathrm{~kg} \cdot \mathrm{m}^{-3}$ (3 $\sigma$ uncertainty). From this density, and considering Camilla's spectral similarities with (24) Themis and (65) Cybele (for which water ice coating on surface grains was reported), we infer a silicate-to-ice mass ratio of 1-6, with a 10-30\% macroporosity. 


\section{Introduction}

Main belt asteroids are the remnants of the building blocks that accreted to form terrestrial planets, leftovers of the dynamical events that shaped our planetary system. Among them, large bodies (diameter larger than $\approx 100 \mathrm{~km}$ ) are deemed primordial (Morbidelli et al., 2009), and contain a relatively pristine record of their initial formation conditions

Decades of photometric and spectroscopic surveys have provided an ever-improving picture of the distribution of material in the inner solar system (e.g. Gradie and Tedesco, 1982; Burbine et al., 1996; 2002; Bus and Binzel, 2002a; Rivkin et al., 2002; 2006; Vernazza et al., 2008; 2010; Vernazza et al., 2014; DeMeo and Carry, 2014), yet these studies have probed the composition of the surface only. As such, they do not necessarily lead us to the original location and time scales for the accretion of these blocks, which are key to understanding the important processes in the disk of gas and dust around the young Sun.

These issues can be addressed by studying the internal structure of asteroids: objects formed far from the Sun are expected to be composed of various mixtures of rock and ice, while objects closer to the Sun are expected to be volatile-free. Depending on their formation time scale, the amount of radiogenic heat varied, leading to complete, partial, or no differentiation. In that respect, density is clearly the most important remotely measurable property that can constrain internal structure (Scheeres et al., 2015).

Determination of density requires measurement of mass and volume, and for that, large asteroids with satellites are prime targets (Merline et al., 1999; 2002; Marchis et al., 2008b; 2008a; Carry et al., 2011; Margot et al., 2015). The study of the orbits of satellites within asteroid binaries or multiple systems is currently the most precise method to estimate the mass of the primary asteroid. If the primary also happen to have an angular diameter large enough to be spatially resolved by large telescopes, this also allows an accurate determination of the primary's volume. In addition, the orbits of the satellites themselves offer a way to probe the gravity field, related to mass distribution inside the asteroid (Berthier et al., 2014; Marchis et al., 2014).

Here we focus on the outer-main-belt asteroid (107) Camilla, orbiting in the Cybele region and discovered on November 17, 1868 from Madras, India by N. R. Pogson. Its first satellite, S/2001 (107) 1 (hereafter S1), was discovered in March 2001 by Storrs et al. (2001), using the Hubble Space Telescope (HST), and its orbit first studied by Marchis et al. (2008a) using observations from large ground-based telescopes equipped with adaptive-optics (AO) systems. Its second satellite, S/2016 (107) 1 (hereafter S2), was discovered in 2016 by our team (Marsset et al., 2016), using the European Southern Observatory (ESO) Very Large Telescope (VLT).

Camilla was originally classified as a C-type based on its visible colors and albedo (Tedesco et al., 1989). Later on, both Bus and

\footnotetext{
Based on observations obtained at: (1) the Hubble Space Telescope, operated by NASA and ESA; (2) the Gemini Observatory and acquired through the Gemini Observatory Archive, which is operated by the Association of Universities for Research in Astronomy, Inc., under a cooperative agreement with the NSF on behalf of the Gemini partnership: the National Science Foundation (United States), the National Research Council (Canada), CONICYT (Chile), Ministerio de Ciencia, Tecnología e Innovación Productiva (Argentina), and Ministério da Ciência, Tecnologia e Inovação (Brazil); (3) the European Southern Observatory, Paranal, Chile - 071.C-0669 (PI Merline), 073.C-0062 and 074.C-0052(PI Marchis), 087.C-0014(PI Marchis), 088.C-0528 (PI Rojo), 095.C-0217 and 297.C-5034 (PI Marsset) - and (4) the W. M. Keck Observatory, which is operated as a scientific partnership among the California Institute of Technology, the University of California and the National Aeronautics and Space Administration. The Observatory was made possible by the generous financial support of the W. M. Keck Foundation.

* Corresponding author at: IMCCE, Observatoire de Paris, PSL Research University, CNRS, Sorbonne Universités, UPMC Univ Paris 06, Univ. Lille, France.

E-mail address: myriam.pajuelo@obspm.fr (M. Pajuelo).
}

Binzel (2002a) and Lazzaro et al. (2004) classified it as X, based on visible spectra. More recently, based on a near-infrared spectrum from NASA IRTF Spex, Lindsay et al. (2015) classified Camilla as either Xe or L.

The physical properties of Camilla have been extensively studied, from its rotation period of 4.8 h (e.g., Weidenschilling et al., 1987; di Martino et al., 1987) to its spin and 3D shape model (Torppa et al., 2003; Ďurech et al., 2011; Hanuš et al., 2013; 2017). Its diameter, however, was poorly constrained, with estimates ranging from $185 \pm 9 \mathrm{~km}$ (Marchis et al., 2006) to $256 \pm 12 \mathrm{~km}$ (Marchis et al., 2012). More recent studies combining images or stellar occultations with lightcurve-based 3D shape modeling, are yielding diameters in excess of $220 \mathrm{~km}$ (see Fig. B.2 and Table B.2 for the exhaustive list of diameter estimates). The mass estimates also spanned a wide range, from $2.25_{-2.25}^{+18.00}$ to $39 \pm 10 \times 10^{18} \mathrm{~kg}$ (Zielenbach, 2011) (see Fig. B.1 and Table B.1 for the exhaustive list of mass estimates). With these large spread of values, deriving an accurate density would require substantial improvements to these parameters.

Gathering all the available disk-resolved and high-contrast images from HST and AO-fed cameras, optical lightcurves, stellar occultations, and visible and near-infrared spectra (Section 2), we present an extensive study of the dynamics of the system (Section 3), of the surface properties of Camilla and its main satellite S1 (Section 4), and of Camilla's spin and 3-D shape (Section 5), all constraining its internal composition and structure (Section 6).

\section{Observations}

\subsection{Optical lightcurves}

We gather the 24 lightcurves used by Torppa et al. (2003) to create a convex 3-D shape model of Camilla ${ }^{1}$, compiled from the Uppsala Asteroid Photometric Catalog ${ }^{2}$ (Lagerkvist and Magnusson, 2011). We also retrieve the three lightcurves reported by Polishook (2009).

In addition to these data, we acquired 29 lightcurves using the $60 \mathrm{~cm}$ André Peyrot telescope mounted at Les Makes observatory on Réunion Island, operated as a partnership among Les Makes Observatory and the IMCCE, Paris Observatory. We also extracted 63 lightcurves from the data archive of the SuperWASP survey (Pollacco et al., 2006) for the period 2006-2009. This survey aims to find and characterize exoplanets by observations of their transits of the host star. Its large field of view $\left(8^{\circ} \times 8^{\circ}\right)$ provides a goldmine for asteroid lightcurves (Parley et al., 2005; Grice et al., 2017).

A total of 127 lightcurves observed between 1981 and 2016 (Table A.1) are used in this work.

\subsection{High-angular-resolution imaging}

We compile here all the high-angular-resolution images of Camilla taken with the HST and large ground-based telescopes equipped with AO-fed cameras: Gemini North, ESO VLT, and W. M. Keck, of which only a subset had already been published (Storrs et al., 2001; Marchis et al., 2008a). All of these data sets were acquired by the authors of this paper. The data comprise 62 different epochs, with multiple images each, spanning 15 years, from March 2001 to August 2016.

The images from the VLT were acquired with both the first generation instrument NACO (NAOS-CONICA, Lenzen et al., 2003;

\footnotetext{
${ }^{1}$ Available on DAMIT (Ďurech et al., 2010): http://astro.troja.mff.cuni.cz/projects/ asteroids3D|

2 http://asteroid.astro.helsinki.fi/apc/asteroids/
} 
Rousset et al., 2003) and SPHERE (Spectro-Polarimetric Highcontrast Exoplanet REsearch, Fusco et al., 2006; Beuzit et al., 2008), the second generation extreme-AO instrument designed for exoplanet detection and characterization. The images taken with SPHERE used its IRDIS differential imaging camera sub-system (InfraRed Dual-band Imager and Spectrograph, Dohlen et al., 2008). Images taken at the Gemini North used NIRI camera (Near InfraRed Imager, Hodapp et al., 2003), fed by the ALTAIR AO system (Herriot et al., 2000). Finally, observations at Keck were acquired with NIRC2 (Near-InfraRed Camera 2, van Dam et al., 2004; Wizinowich et al., 2000). We list in Table A.2 the details of each observation.

The basic data processing (sky subtraction, bad-pixel removal, and flat-field correction) was performed using in-house routines developed in Interactive Data Language (IDL) to reduce AO-imaging data (see Carry et al., 2008, for more details).

\subsection{High-angular-resolution spectro-imaging}

In 2015 and 2016, we also used the integral-field spectrograph (IFS) of the SPHERE instrument at the ESO VLT, aiming to measure the reflectance spectrum of Camilla's largest satellite S1, and the astrometry of the fainter satellite S2. The observations were made in the IRDIFS_EXT mode (Zurlo et al., 2014), in which both IRDIS (Dohlen et al., 2008) and the IFS (Claudi et al., 2008) data are acquired simultaneously. In this set-up, the IFS covers the wavelength range from 0.95 to $1.65 \mu \mathrm{m}$ (YJH bands) at a spectral resolving power of $\sim 30$ in a $1.7^{\prime \prime} \times 1.7^{\prime \prime}$ field of view (FoV), while IRDIS operates in the dual-band imaging mode (DBI, Vigan et al., 2010) with $K_{12}$, a pair of filters in the $K$ band $\left(\lambda_{K_{1}}=2.110 \mu \mathrm{m}\right.$ and $\lambda_{K_{2}}=2.251 \mu \mathrm{m}, \sim 0.1 \mu \mathrm{m}$ bandwidth), within a $4.5^{\prime \prime}$ FoV. All observations were performed in the pupil-tracking mode, where the pupil remains fixed while the field orientation varies during the observations. This mode provides the best PSF stability and helps in reducing and subtracting static speckle noise in the images.

For the pre-processing of both the IFS and IRDIS data, we used the preliminary release (v0.14.0-2) of the SPHERE Data Reduction and Handling (DRH) software (Pavlov et al., 2008), as well as additional in-house tools written in IDL, including parts of the public pipeline presented in Vigan et al. (2015). See our recent works on (3) Juno and (6) Hebe for more details (Viikinkoski et al., 2015; Marsset et al., 2017). We used the DRH for the creation of some of the basic calibrations: master sky frames, master flat-field, IRDIFS spectra positions, initial wavelength calibration and flat field. Before creating the data cubes, we used IDL routines to subtract the background from each science frame and correct for the bad pixels identified using the master dark and master flat-field DRH products. This step was introduced as a substitute for the bad pixel correction provided by the DRH. Bad pixels were first identified using a sigma-clipping routine, and then corrected using a bicubic pixel interpolation with the MASKINTERP IDL routine. The resulting frames were then injected into the DRH recipe to create the data cubes by interpolating the data spectrally and spatially.

\subsection{Stellar occultations}

Eleven stellar occultations by Camilla have been observed in the last decade, mostly by amateur astronomers (see Mousis et al., 2014; Dunham et al., 2016a). The timings of disappearance and reappearance of the stars, together with the location of each observing station are compiled by Dunham et al. (2016b), and publicly available on the Planetary Data System $\left(\mathrm{PDS}^{3}\right)$. We converted the disappearance and reappearance timings (Table A.3) of the occulted stars into segments (called chords) on the plane of the sky,

\footnotetext{
3 http://sbn.psi.edu/pds/resource/occ.html
}

using the location of the observers on Earth and the apparent motion of Camilla following the recipes by Berthier (1999). Four stellar occultations had multiple chords; other events had only one or two positive chords, and contributed less to constraining the size and apparent shape of Camilla. In none of these eleven stellar occultations was there any evidence for a companion. We list in Table A. 4 the details of the seven events that we used.

\subsection{Near-infrared spectroscopy}

On November 1, 2010, we observed Camilla over 0.8-2.5 $\mu \mathrm{m}$ with the near-infrared spectrograph SpeX (Rayner et al., 2003), on the 3-meter NASA IRTF located on Mauna Kea, Hawaii, using the low resolution Prism mode $(R=100)$. We used the standard nodding procedure for the observations, using alternately two separated locations on the slit (e.g., Nedelcu et al., 2007) to estimate the sky background. We used Spextool (SPectral EXtraction TOOL), an IDL-based data reduction package written by Cushing et al. (2004) to reduce SpeX data.

\section{Dynamical properties}

\subsection{Data processing}

The main challenges in measuring the position and apparent flux of the satellite of an asteroid results from their sub-arcsecond angular separation and high contrast (several magnitudes), combined with imperfect AO correction. A typical image of a binary asteroid (Fig. 1) displays a central peak (the asteroid itself, angularly resolved or not) encompassed by a halo (its diffused light), within which speckle patterns appear. The faintness of these speckles, produced by interference of the incoming light, make them very similar in appearance to a small moon with a contrast up to several thousands, and they can be misleading. Speckles, however, vary (position and flux) on short timescales, depending on the ambient conditions and AO performances (e.g., seeing, airmass, brightness of the AO reference source). These fluctuations can be used to distinguish genuine satellites from speckles.

As for the direct imaging of exoplanets, it is crucial to substract the halo that surrounds the primary (in a similar way to the digital coronography of Assafin et al., 2008). Because asteroids are also marginally resolved, their light is not fully coherent, and the speckle pattern is not as stable in time, nor simple, as in the case of a star. The tool we developed considers concentric annuli around the center of light of the primary to evaluate its halo. Although the principle is straightforward, great caution was taken in the implementation, especially in the computation of the intersection of the annulus with the pixels to allow the use of annuli with a sub-pixel width. The contribution of each pixel to different annuli is thus solved first, and the median flux of each annulus is computed, and subtracted from each pixel accordingly.

The position and flux of the satellite, relative to the primary, is then measured by fitting a 2-D Gaussian function to the halosubtracted image. The satellites are distinguished from speckles by comparing different images, taken both close in time and over a range of times. To estimate the uncertainties on the position and apparent flux of both the primary and the satellites, we use different integration apertures for each object. The sizes of the apertures are determined by fitting a 2-D Gaussian to each, with diameters typically being 5 to 150 pixels for the primary, and 3 to 15 pixels for the satellites. The reported positions and apparent magnitudes (Tables A.5 and A.6) are the average of all fits (after removal of outlier values), and the reported uncertainties are the standard deviations. 


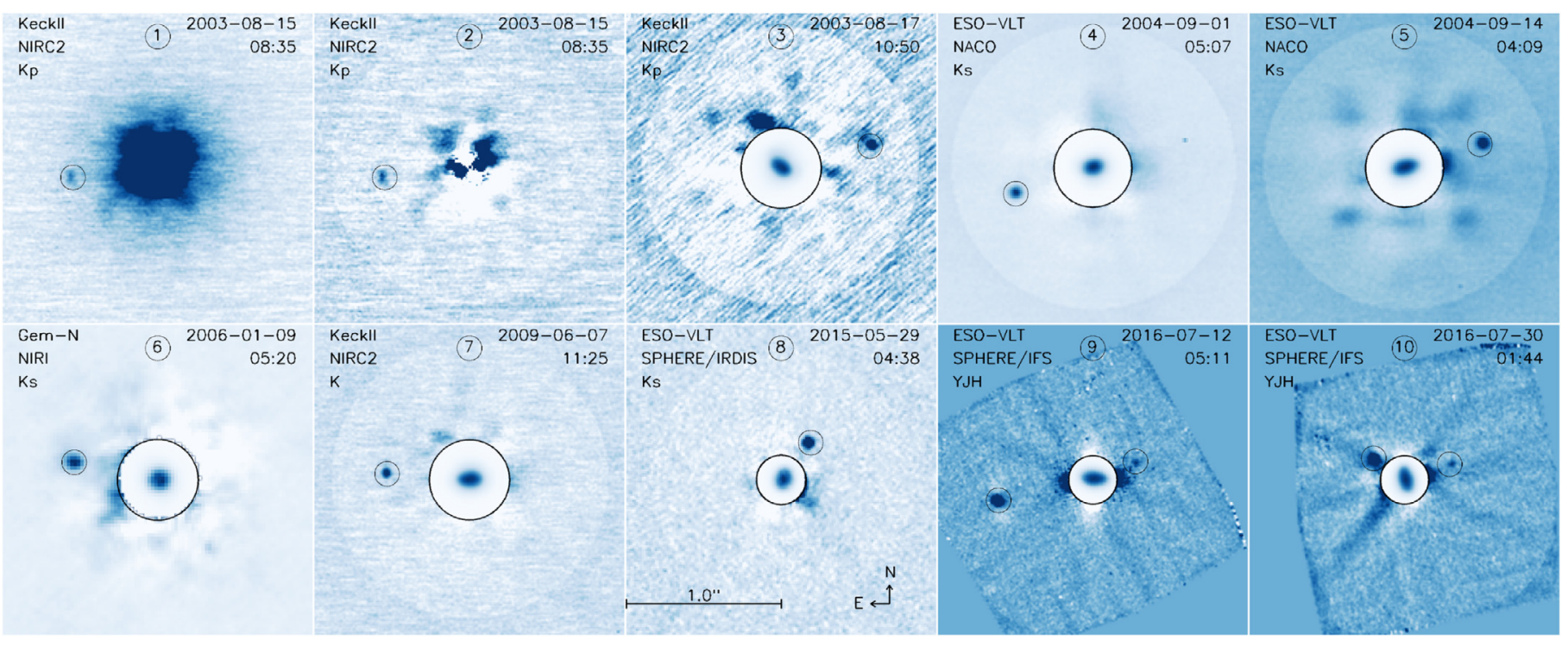

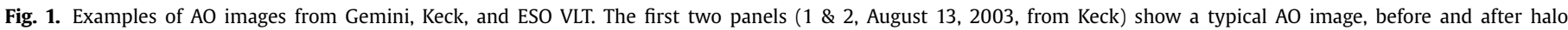

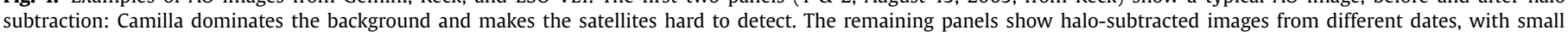

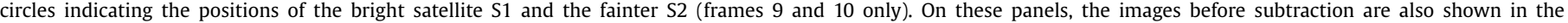
central circle to highlight the elongated shape of Camilla.

\subsection{Orbit determination with Genoid}

We use our algorithm Genoid (GENetic Orbit IDentification, Vachier et al., 2012) to determine the orbit of the satellites. Genoid is a genetic-based algorithm that relies on a metaheuristic method to find the best-fit (i.e., minimum $\chi^{2}$ ) suite of dynamical parameters (mass, semi-major axis, eccentricity, inclination, longitude of the node, argument of pericenter, and time of passage to pericenter) by refining, generation after generation, a grid of test values (called individuals).

The first generation is drawn randomly over a very wide range for each parameter, thus avoiding a miss of the global minimum from inadequate initial conditions. For each individual (i.e., set of dynamical parameters), the $\chi^{2}$ residuals between the observed and predicted positions is computed as

$\chi^{2}=\sum_{i=1}^{N}\left[\left(\frac{X_{o, i}-X_{c, i}}{\sigma_{x, i}}\right)^{2}+\left(\frac{Y_{o, i}-Y_{c, i}}{\sigma_{y, i}}\right)^{2}\right]$

where $N$ is the number of observations, and $X_{i}$ and $Y_{i}$ are the relative positions between the satellite and Camilla along the right ascension and declination respectively. The indices $o$ and $c$ stand for observed and computed positions, and $\sigma$ are the measurement uncertainties.

A new generation of individuals is drawn by mixing randomly the parameters of individuals with the lowest $\chi^{2}$ from the former generation. This way, the entire parameter space is scanned, with the density of evaluation points increasing toward low $\chi^{2}$ regions along the process. At each generation, we also use the best individual as initial condition to search for the local minimum by gradient descent. The combination of genetic grid-search and gradient descent thus ensures finding the best solution.

We then assess the confidence interval of the dynamical parameters by considering all the individuals providing predictions within 1,2 , and $3 \sigma$ of the observations. The range spanned by these individuals provide the confidence interval at the corresponding $\sigma$ level for each parameter.

The reliability of Genoid has been assessed during a stellar occultation by (87) Sylvia and its satellites Romulus and Remus on January 6, 2013: Genoid had been used to predict the position of
Romulus before the event, directing observers to locations specifically to target the satellite. Four different observers detected an occultation by Romulus at only $13.5 \mathrm{~km}$ off the predicted track (the cross-track uncertainty was $65 \mathrm{~km}$, Berthier et al., 2014).

\subsection{Orbit of S1: S/2001 (107) 1}

We measured 80 astrometric positions of the satellite S1 relative to Camilla over a span of 15 years, corresponding to 5642 days or 1520 revolutions. The orbit we derive with Genoid fits all 80 observed positions of the satellite with a root mean square (RMS) residual of 7.8 milli-arcseconds (mas) only, which corresponds to a sub-pixel accuracy.

S1 orbits Camilla on a circular, prograde, equatorial orbit, in 3.71 days with a semi-major axis of $1248 \mathrm{~km}$. We detail all the parameters of its orbit in Table 1, with their confidence interval taken at $3 \sigma$. The distribution of residuals between the observed and predicted positions, normalized by the uncertainty on the measured positions, are plotted in Fig. 2. The orbit we determine here is qualitatively similar to the one given by Marchis et al. (2008a), while much better constrained: we fit 80 astrometric positions over 15 years with an RMS residual of 7.8 mas, compared to their fit of 23 positions over less than 3 years with an RMS residual of 22 mas. The much longer time span of observations provides a much more stringent constraint on the period (3.712 $34 \pm 0.00004$ day) of S1, compared to the value of $3.722 \pm 0.009$ day reported by Marchis et al. (2008a).

As a result, we determine a much more precise mass for Camilla of $(1.12 \pm 0.01) \times 10^{19} \mathrm{~kg}$ (3 $\sigma$ uncertainty), about $1 \%$ of the mass of Ceres (Carry, 2012). We list in Table B.1 the reported values of the mass of Camilla found in the literature. Our mass value agrees well with the average value $(1.10 \pm 0.69) \times 10^{19} \mathrm{~kg}$ we show in Table B.1, although the mass estimates derived from orbital deflection and solar system ephemerides have a large scatter (see Carry, 2012, for a discussion on the precision and bias of mass determination methods). Our determination significantly reduces the uncertainty in the prior value of $(1.12 \pm 0.09) \times 10^{19} \mathrm{~kg}$, that also used the orbit of S1 (Marchis et al., 2008a). 
Table 1

Orbital elements of the satellites of Camilla, S1 and S2, expressed in EQJ2000, obtained with Genoid: orbital period $P$, semi-major axis $a$, eccentricity $e$, inclination $i$, longitude of the ascending node $\Omega$, argument of pericenter $\omega$, time of pericenter $t_{p}$. The number of observations and RMS between predicted and observed positions are also provided. Finally, we report the derived primary mass $M$, the ecliptic J2000 coordinates of the orbital pole $\left(\lambda_{p}, \beta_{p}\right)$, the equatorial $\mathrm{J} 2000$ coordinates of the orbital pole $\left(\alpha_{p}, \delta_{p}\right)$, and the orbital inclination $(\Lambda)$ with respect to the equator of Camilla. Uncertainties are given at $3-\sigma$.

\begin{tabular}{|c|c|c|c|c|}
\hline & $\mathrm{S} 1$ & & $\mathrm{~S} 2$ & \\
\hline \multicolumn{5}{|c|}{ Observing data set } \\
\hline Number of observations & 80 & & 11 & \\
\hline Time span (days) & 5642 & & 428 & \\
\hline RMS (mas) & 7.8 & & 5.0 & \\
\hline \multicolumn{5}{|c|}{ Orbital elements EQJ2000 } \\
\hline$P$ (day) & 3.71234 & \pm 0.00004 & 1.376 & \pm 0.016 \\
\hline$a(\mathrm{~km})$ & 1247.8 & \pm 3.8 & 643.8 & \pm 3.9 \\
\hline$e$ & 0.0 & +0.013 & 0.18 & ${ }_{-0.18}^{+0.23}$ \\
\hline$i\left({ }^{\circ}\right)$ & 16.0 & \pm 2.3 & 27.7 & $\begin{array}{l}-0.18 \\
\pm 21.8\end{array}$ \\
\hline$\Omega\left({ }^{\circ}\right)$ & 140.1 & \pm 4.9 & 219.9 & \pm 67.0 \\
\hline$\omega\left(^{\circ}\right)$ & 98.7 & \pm 6.5 & 199.4 & \pm 37.6 \\
\hline$t_{p}(\mathrm{JD})$ & 2452835.902 & \pm 0.067 & 2452835.31589 & \pm 0.174 \\
\hline \multicolumn{5}{|c|}{ Derived parameters } \\
\hline$M\left(\times 10^{19} \mathrm{~kg}\right)$ & 1.12 & \pm 0.01 & & \\
\hline$\lambda_{p}, \beta_{p}\left(^{\circ}\right)$ & $73,+53$ & $\pm 2,2$ & $114,+42$ & $\pm 44,18$ \\
\hline$\alpha_{p}, \delta_{p}\left({ }^{\circ}\right)$ & $50,+74$ & $\pm 5,2$ & $130,+62$ & $\pm 67,22$ \\
\hline$\Lambda\left({ }^{\circ}\right)$ & 4 & \pm 8 & 32 & \pm 28 \\
\hline
\end{tabular}

\subsection{Orbit of S2: S/2016 (107) 1}

We measured 11 astrometric positions of the satellite S2 relative to Camilla during 2015 and 2016, corresponding to 428 days or 311 revolutions. These observations correspond to three wellseparated epochs: 2015-May-29, 2016-Jul-12, and 2016-Jul-30, providing the minimum needed to constrain the orbit. Thus, although the orbit we determine with Genoidfits all 11 observed positions of S2 with an RMS residual of only 5.0 mas and yields reliable values for the major orbital elements, details of all orbital parameters will require further observations.

S2 orbits Camilla in 1.38 days with a semi-major axis of $644 \mathrm{~km}$. We detail all the parameters of its orbit in Table 1 and present the distribution of residuals between the observed and predicted positions in Fig. 3. Unlike S1, its orbit seems neither equatorial nor circular. While cognizant of the larger uncertainties, we favor an orbit inclined to the equator of Camilla by an angle $\Lambda$ of $32 \pm 28^{\circ}$ (Fig. 4), and a more eccentric orbit $\left(\mathrm{e}=0.18_{-0.18}^{+0.23}\right.$ ). Although a circular orbit, co-planar with $\mathrm{S} 1$ is marginally within the range of uncertainty, such a solution results in significantly higher residuals. This configuration of an outer satellite on a circular and equatorial orbit with an inner satellite on an inclined and more eccentric orbit has already been reported for other triple systems: (45) Eugenia, (87) Sylvia, and (130) Elektra (Marchis et al., 2010; Fang et al., 2012; Berthier et al., 2014; Yang et al., 2016; Drummond et al., 2016).

\section{Surface properties}

\subsection{Data processing}

We measured the near-infrared spectra of Camilla and its largest satellite S1 using the SPHERE/IRDIFS data. Telluric features were removed, and the reflectance spectra were obtained by observing the nearby solar type star HD139380.

Similarly to previous sections, the bright halo of Camilla that contaminated the spectrum of the moon was removed. This was achieved by measuring the background at the location of the moon for each pixel as the median value of the area defined as a $40 \times 1$-pixel arc centered on Camilla. To estimate the uncertainty and potential bias on photometry introduced by this

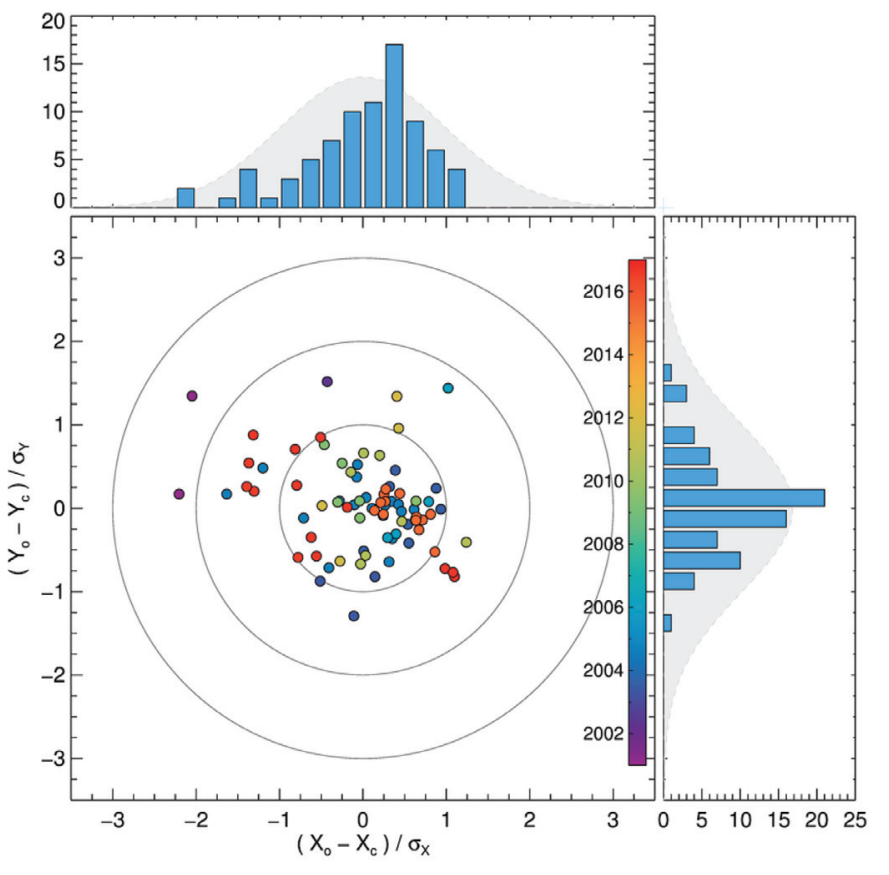

Fig. 2. Distribution of residuals for $\mathrm{S} 1$ between the observed (index o) and predicted (index c) positions, normalized by the uncertainty on the measured positions $(\sigma)$, and color-coded by observing epoch. X stands for right ascension and $\mathrm{Y}$ for declination. The three large gray circles represent the 1,2 , and $3 \sigma$ limits. The top panel shows the histogram of residuals along $\mathrm{X}$, and the right panel the residuals along Y. The light gray Gaussian in the background has a standard deviation of one.

method, we performed a number of simulations in which we injected fake companions on the 39 spectral images of the spectroimaging cube, at separation ( $\approx 300$ mas) and random position angles from the primary. The simulated sources were modeled as the PSF, from the calibration star images, scaled in brightness.

The halo from Camilla was then removed from these simulated images using the method described above, and the flux of the simulated companion measured by adjusting a 2D-Gaussian profile. Based on a total statistics of 500 simulated companions, we 


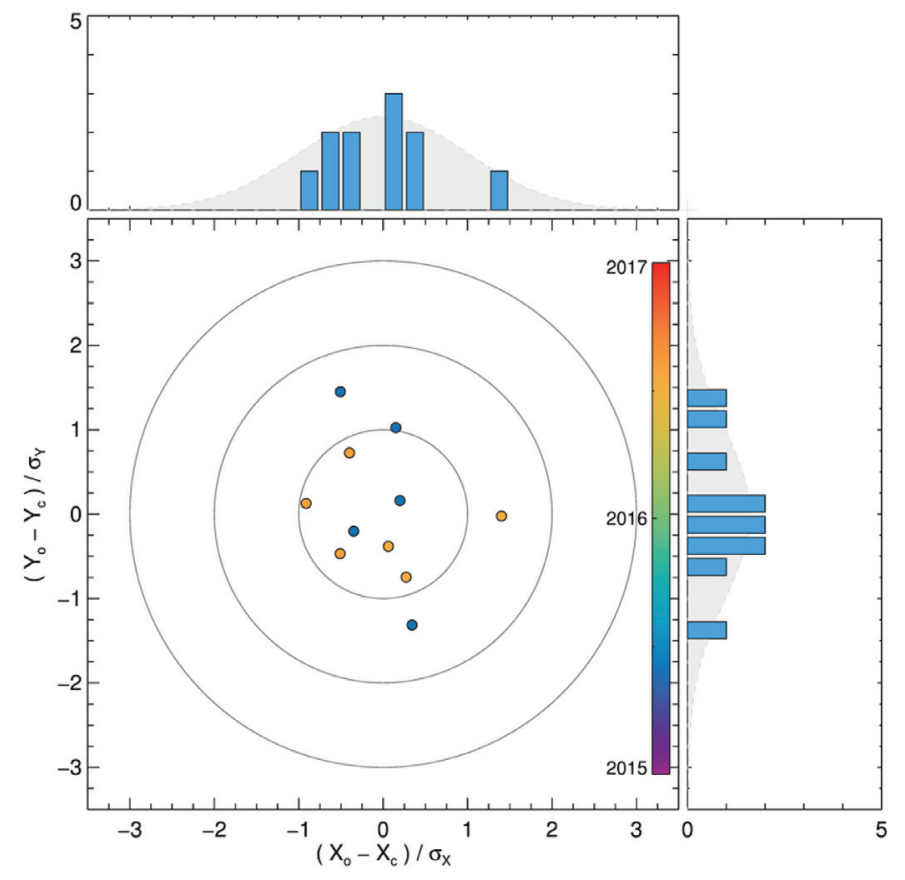

Fig. 3. Similar to Fig. 2, but for S2.

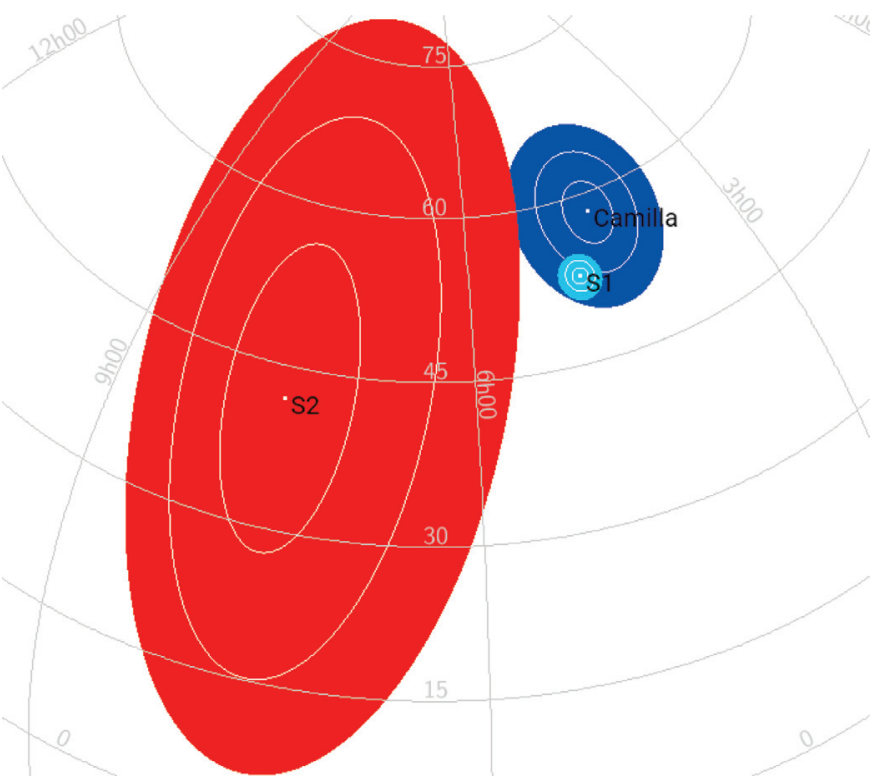

Fig. 4. Coordinates and $1-2-3 \sigma$ contours of Camilla's spin axis (blue) and the orbital poles of S1 (gray) and S2 (red) in ecliptic coordinates. (For interpretation of the references to colour in this figure legend, the reader is referred to the web version of this article.)

find that the median loss of flux at each wavelength is $11 \pm 10 \%$. A spectral gradient is also introduced by our technique, but it is smaller than $0.06 \pm 0.07 \% \cdot \mu \mathrm{m}^{-1}$. The spectra of Camilla and S1, normalized at $1.1 \mu \mathrm{m}$, are shown in Fig. 5.

\subsection{Spectrum of Camilla}

We combine the near-infrared spectrum we acquired at NASA IRTF (Section 2.5) with the visible spectrum from SMASS (Bus and Binzel, 2002a; 2002b) and analyze them with the M4AST ${ }^{4}$ (Model-

\footnotetext{
4 http://m4ast.imcce.fr
}

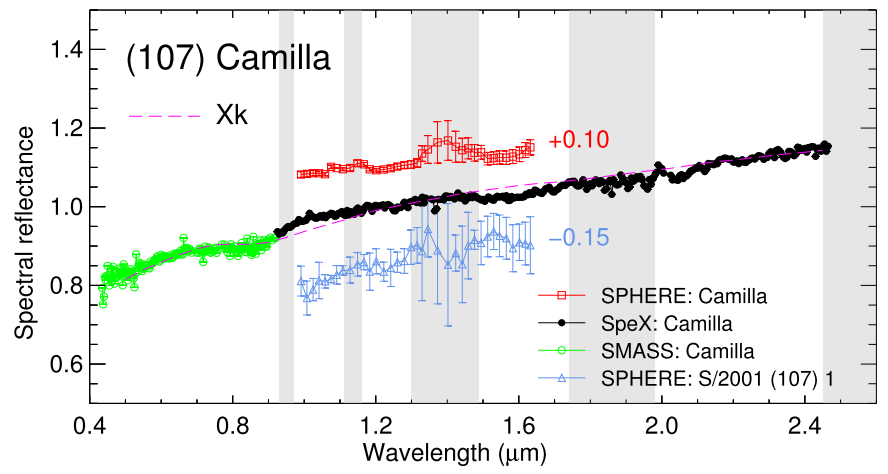

Fig. 5. Visible and near-infrared spectrum of Camilla from IRTF (green and black dots) and SPHERE (red squares, offset by +0.1 ), and its moon S1 from SPHERE (blue triangles, offset by -0.15 ). Gray areas represent the wavelength ranges affected by water vapour in the atmosphere. All spectra were normalized to unity at one micron. Overplot to the IRTF spectra is the Bus-DeMeo Xk class. (For interpretation of the references to color in this figure legend, the reader is referred to the web version of this article.)

ing for Asteroids, Popescu et al., 2012) suite of Web tools to determine asteroid taxonomic classification, mineralogy, and most-likely meteorite analog. From this longer wavelength range, we found Camilla to be an Xk-type asteroid (using Bus-DeMeo taxonomic scheme, Fig. 5, DeMeo et al., 2009). The low albedo of Camilla $(0.059 \pm 0.005$, taken as the average of the estimates by Morrison and Zellner, 2007; Tedesco et al., 2002; Ryan and Woodward, 2010; Usui et al., 2011; Masiero et al., 2011), hints at a P-type classification, using the Tedesco et al. (1989) scheme.

Although the best spectral match is formally found for an Enstatite Chondrite EH5 meteorite (Queen Alexandra Range, Antarctica origin, maximum size of $10 \mu \mathrm{m}$ ), the low albedo of Camilla argues for a different type of analog material. The composition of P-type asteroids is indeed difficult, if not impossible, to infer from their visible and near-infrared spectra owing to the lack of absorption bands.

Recently, Vernazza et al. (2015) have shown that anhydrous chondritic porous interplanetary dust particules (IDPs) were likely to originate from D- and P-types asteroids, based on spectroscopic observations in the mid-infrared of outer-belt D- and P-type asteroids, including Camilla. The mixture of olivine-rich and pyroxenerich IDPs they used was compatible with the visible and nearinfrared spectrum of Camilla. As such, the surface of Camilla, and more generally of D- and P-types, is very similar to that of comets, as already reported by Emery et al. (2006) from the spectroscopy of Jupiter Trojans in the mid-infrared, revealing the presence of anhydrous silicates.

\subsection{Spectrum of $S 1$}

As visible in Fig. 5, the spectrum of S1 is similar to that of Camilla. No significant difference in slope nor absorption band can be detected. This implies that the two components are spectraly identical from 0.95 to $1.65 \mu \mathrm{m}$, within the precision of our measurements. Such a similarity between the components of multiple systems have already been reported for several other main-belt asteroids: (22) Kalliope (Laver et al., 2009), (90) Antiope (Polishook et al., 2009; Marchis et al., 2011), (130) Elektra (Yang et al., 2016), and (379) Huenna (DeMeo et al., 2011).

Such spectral similarity, together with the main characteristics of the orbit (prograde, equatorial, circular) supports an origin of these satellites, here for S1 in particular, by impact and reaccumulation of material in orbit (see Margot et al., 2015, for a review). Formation by rotational fission is unlikely owing to the rotation period of Camilla (4.84 h). 


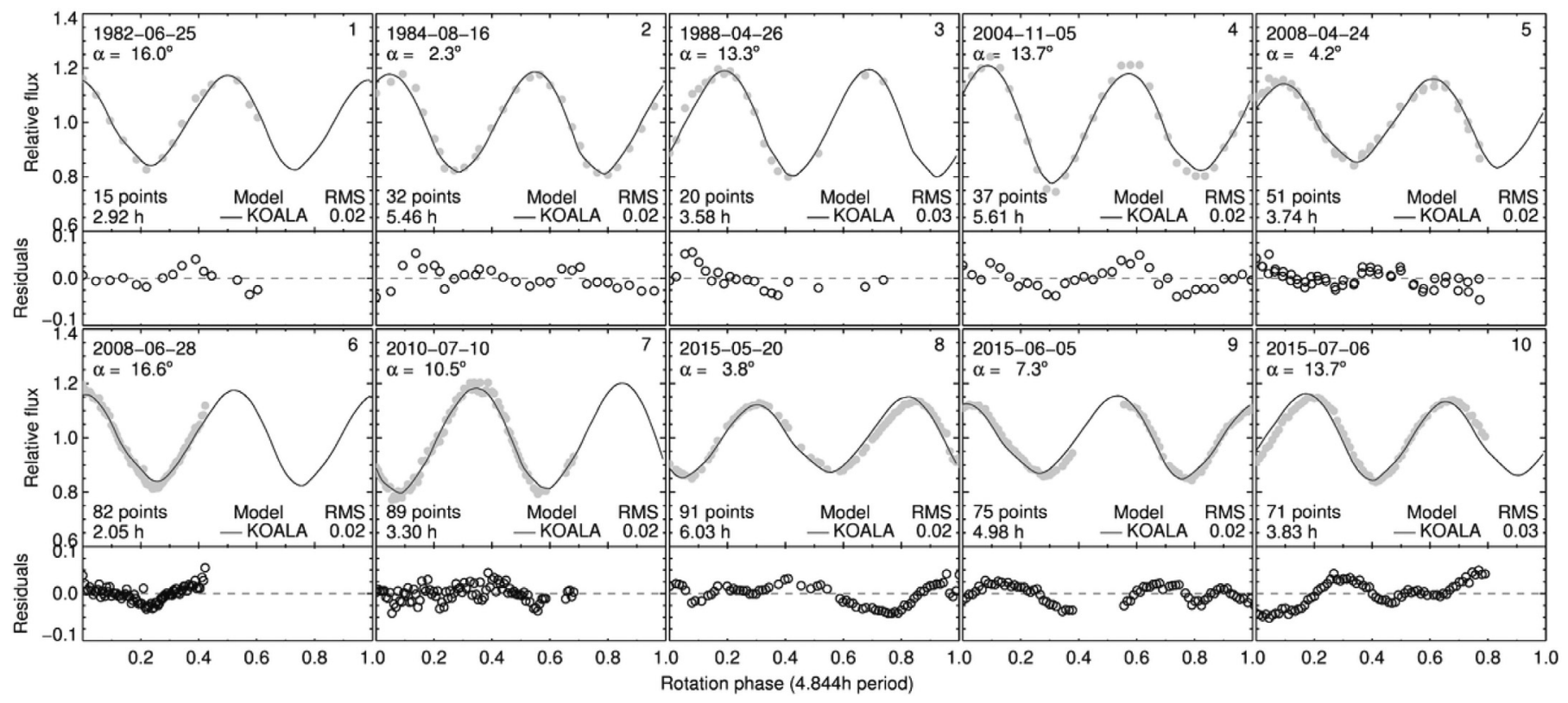

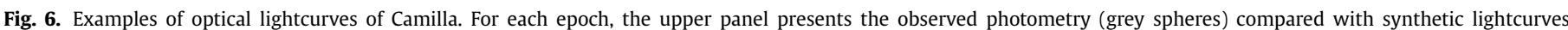

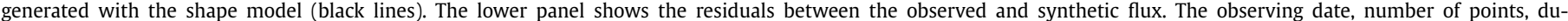

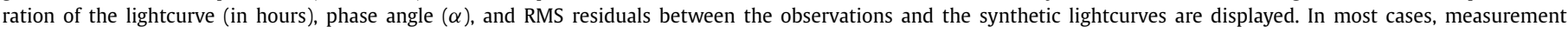
uncertainties are not provided by the observers but can be estimated from the spread of measurements. See Fig. C.1 for the entire data set.

\section{Physical properties}

\subsection{Data processing}

We used the optical lightcurves without modification, only converting their heterogeneous formats from many observers to the usual lightcurve inversion format (Ďurech et al., 2010). For the occultation observations, the location of observers, together with their timings of the disappearance and the reappearance of the star, were converted into chords on the plane of the sky, using the recipes from Berthier (1999). Finally, the 2-D profile of the apparent disk of Camilla was measured on the AO images, deconvolved using the Mistral algorithm (Fusco, 2000; Mugnier et al., 2004), the reliability of which has been demonstrated elsewhere (Witasse et al., 2006), using the wavelet transform described in Carry et al. (2008, 2010b).

\subsection{3-D Shape modeling with KOALA}

We used the multi-data inversion algorithm Knitted Occultation, Adaptive-optics, and Lightcurve Analysis (KOALA), which determines the set of rotation period, spin-vector coordinates, and 3-D shape that provide the best fit to all observations simultaneously (Carry et al., 2010a).

The KOALA algorithm minimizes the total $\chi^{2}=\chi_{I C}^{2}+$ $w_{A O} \chi_{A O}^{2}+w_{O c c} \chi_{O c c}^{2}$ that is composed of the individual contributions from light curves (LC), profiles from disk-resolved images (AO), and occultation chords (Occ). Adaptive optics and occultation data are weighted with respect to the lightcurves with parameters $w_{A O}$ and $w_{O c c}$, respectively. Within each type of data, all the epochs are weighted uniformly. The optimum values of these weights can be objectively obtained following the approach of Kaasalainen (2011).

This method has been spectacularly validated by the images taken by the OSIRIS camera on-board the ESA Rosetta mission during its flyby of the asteroid (21) Lutetia (Sierks et al., 2011). Before the encounter, the spin and 3-D shape of Lutetia had been deter- mined with KOALA, using lightcurves and AO images (Carry et al., 2010b; Drummond et al., 2010). A comparison of the pre-flyby solution with the OSIRIS images showed that the spin vectorwas accurate to within $2^{\circ}$ and the diameter to within $2 \%$. The RMS residual in the surface topography between the KOALA predictions and the OSIRIS images was only $2 \mathrm{~km}$, for a $98 \mathrm{~km}$-diameter asteroid (Carry et al., 2012).

\subsection{Spin and 3-D shape of Camilla}

We used 127 optical lightcurves, 34 profiles from disk-resolved imaging, and 7 stellar occultation events to reconstruct the spin axis and 3-D shape of Camilla. The model fits well the entire data set, with mean residuals of only $0.03 \mathrm{mag}$ for the lightcurves (Figs. 6 and C.1), 0.29 pixel for the images (Fig. 7), and $0.35 \mathrm{~s}$ for the stellar occultations (Fig. 8). There are small local departures of the shape model from the stellar occultation chords that can be due to local topography not modeled with our low-resolution shape model.

The rotation period and coordinates of the spin axis (Table 2) agree very well with previous results from lightcurve-only inversion and convex shape modeling (Torppa et al., 2003; Ďurech et al., 2011; Hanuš et al., 2016), as well as models obtained by combining lightcurves and smaller subsets of the present AO data (respectively 3 and 21 epochs, see Hanuš et al., 2013; 2017). The shape of Camilla is far from a sphere, with a strong ellipsoidal elongation along the equator $(\mathrm{a} / \mathrm{b}$ axes ratio of $1.37 \pm 0.12$, see Table 2$)$. Departures from the ellipsoid are, however, limited, and mainly consist in two large circular basins, reminiscent of impact craters (Fig. 9).

The spherical-volume-equivalent diameter of Camilla is found to be $254 \pm 36 \mathrm{~km}(3 \sigma)$, in excellent agreement with the recent determination by Hanuš et al. (2017) based on a similar data set. Both estimates are high compared to diameter estimates from infrared observations with IRAS, AKARI, or WISE (Tedesco et al., 2004; Ryan and Woodward, 2010; Usui et al., 2011; Masiero et al., 2011, see Table B.2). However, diameter determinations by midIR radiometry are based on disk-integrated fluxes. In the case of 


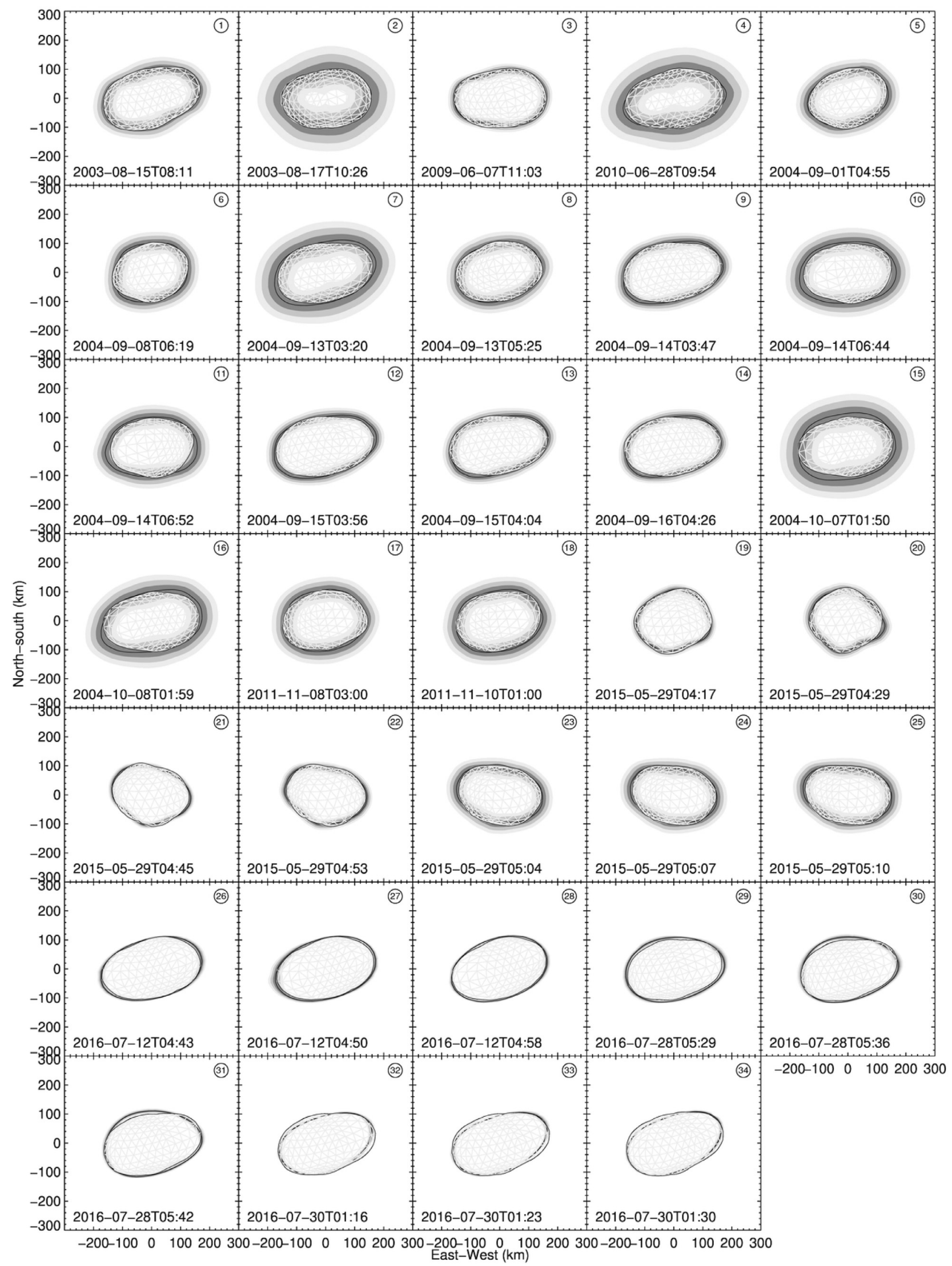

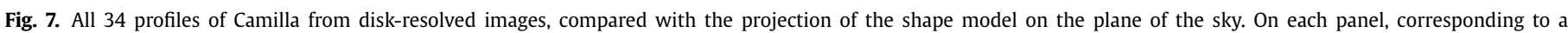
different epoch, the grey shaded areas correspond to the $1-2-3 \sigma$ confidence intervals of each profile, while the shape model is represented by the wired mesh. 


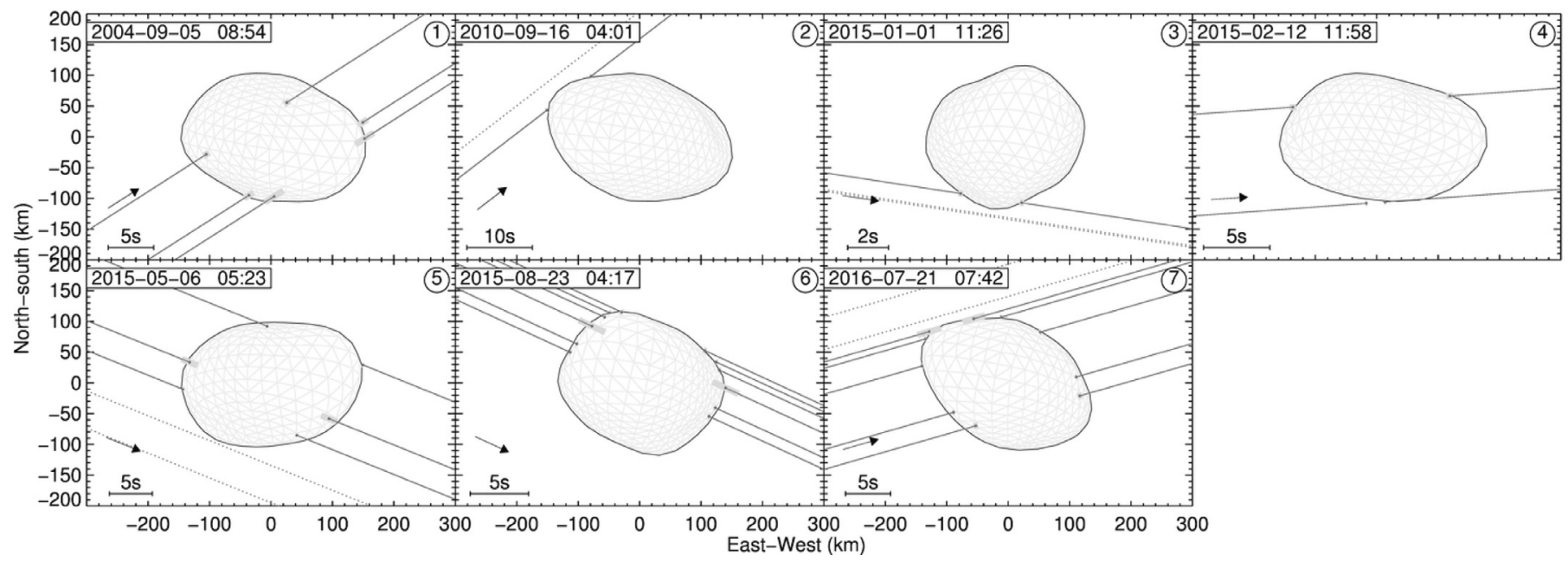

Fig. 8. The seven stellar occultations by Camilla, compared with the shape model projected on the plane of the sky for the times of the occultations. The observer of northern chord in the first occultation, presenting a clear mismatch with the shape model, reported the presence of thin cirrus that may explain the discrepancy.

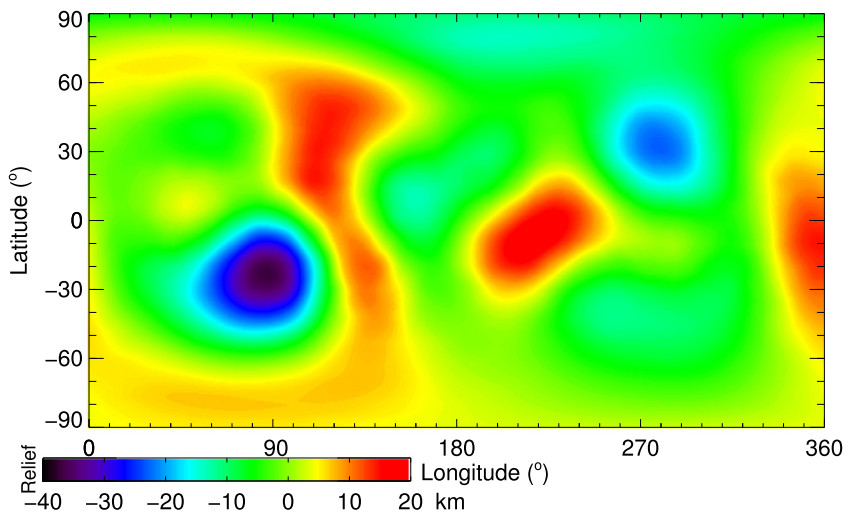

Fig. 9. Topographic map of Camilla, with respect to its reference ellipsoid (Table 2) The main features are the two deep and circular basins located at $\left(87^{\circ},-23^{\circ}\right)$ and $\left(278^{\circ}+33^{\circ}\right)$.

Table 2

Sidereal rotation period, spin-vector coordinates (longitude $\lambda$, latitude $\beta$ in ECJ2000; and right ascension $\alpha$, declination $\delta$ in EQJ2000), sphericalvolume-equivalent diameter (D), volume (V), diameters along the principal axis of inertia (a, b, c), and axes ratio of Camilla obtained with KOALA. All uncertainties are reported at $3 \sigma$.

\begin{tabular}{llll}
\hline Parameter & Value & Unc. & Unit \\
\hline Period & 4.843927 & $4.10^{-5}$ & hour \\
$\lambda$ & 68.0 & 9.0 & deg. \\
$\beta$ & 58.3 & 7.0 & deg. \\
$\alpha$ & 35.8 & 9.0 & deg. \\
$\delta$ & 76.1 & 7.0 & deg. \\
$\mathrm{T}_{0}$ & 2444636.00 & & \\
$\mathrm{D}$ & 254 & 36 & $\mathrm{~km}^{3}$ \\
$\mathrm{~V}$ & $8.55 \cdot 10^{6}$ & $1.21 \cdot 10^{6}$ & $\mathrm{~km}^{3}$ \\
$\mathrm{a}$ & 340 & 36 & $\mathrm{~km}$ \\
$\mathrm{~b}$ & 249 & 36 & $\mathrm{~km}$ \\
$\mathrm{c}$ & 197 & 36 & $\mathrm{~km}$ \\
$\mathrm{a} / \mathrm{b}$ & 1.37 & 0.12 & \\
$\mathrm{~b} / \mathrm{c}$ & 1.26 & 0.12 & \\
\hline
\end{tabular}

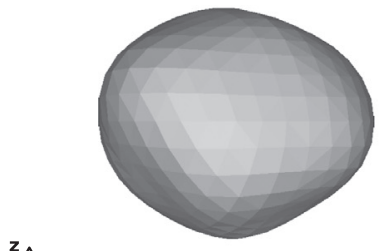<smiles>[R]C(O)[Te]</smiles>
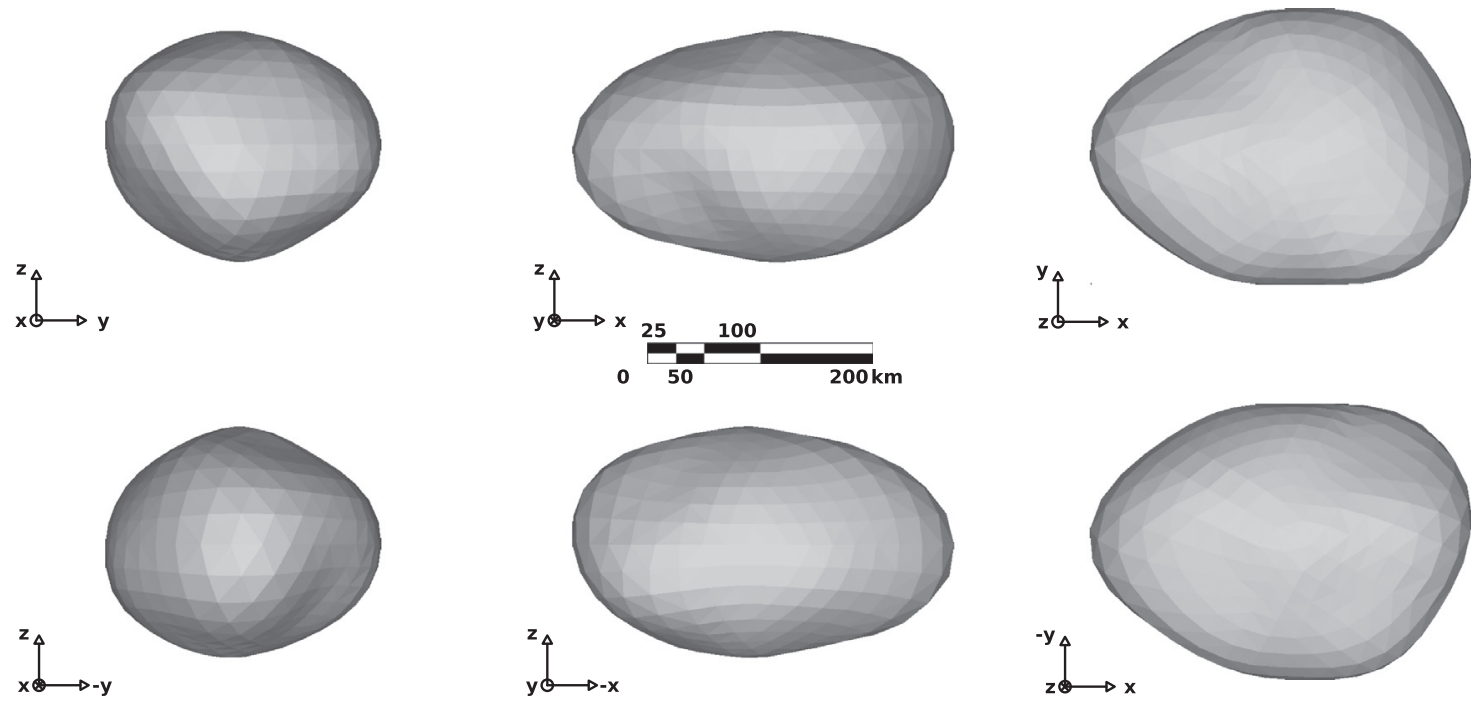

Fig. 10. Views of the shape model along its principal axes (the $x, y, z$ axes in the plot are aligned with the principal moment of inertia of the model). 


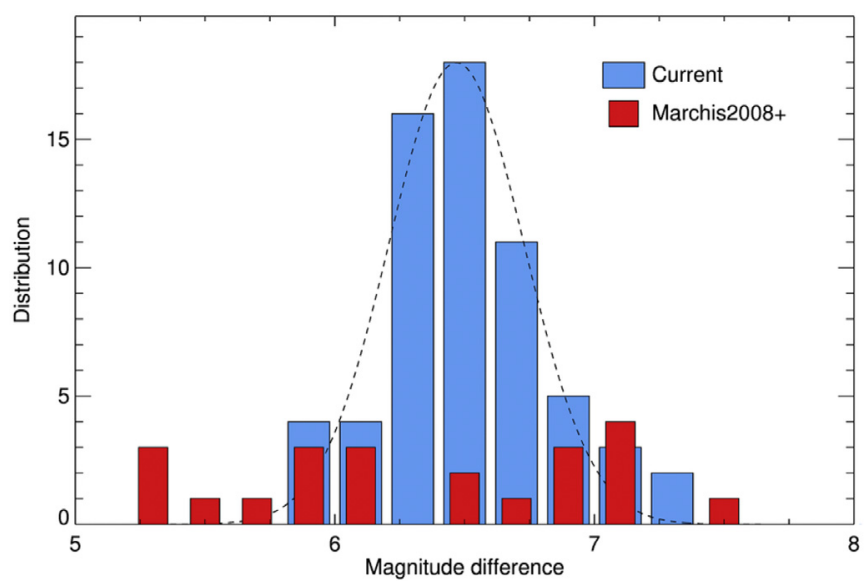

Fig. 11. Distribution of the magnitude differences between Camilla and its largest satellite S1, compared with previous report from Marchis et al. (2008a). The dashed black line represents the normal distribution fit to our results, with a mean and standard deviation of $6.51 \pm 0.27$.

highly elongated targets like Camilla, the projected area is often smaller than the average area as shown in Table B.3. Averaging disk-integrated fluxes may thus underestimate the average diameter.

The agreement of the 3-D models by Hanuš et al. (2017) and developed here with lightcurves, disk-resolved images, and stellar occultation timings, providing direct size measurements, indeed argues for Camilla being larger than previously thought. The corresponding volume is $8.5 \pm 1.2 \cdot 10^{6} \mathrm{~km}^{3}$. The uncertainty on the volume matches closely that of the diameter $(\delta V / V \approx \delta D / D)$ in the case of 3-D shape modeling, as shown by Kaasalainen and Viikinkoski (2012), because it derives from the uncertainty on the radius of each vertex, which are correlated (unlike in the case of scaling a sphere).

\subsection{Diameter of $S 1$}

We list in Table A.5 and display in Fig. 11 the 65 measured brightness differences with an uncertainty lower than 1 magnitude between Camilla and its largest satellite S1. We found a normal distribution of measurement, as expected from photon noise, and measure an average magnitude difference of $\Delta m=6.51 \pm 0.27$, similar to the value of $6.31 \pm 0.68$ reported by Marchis et al. (2008a) on 22 epochs.

Using the diameter of $254 \pm 36 \mathrm{~km}$ for Camilla (Section 5.3) and assuming S1 has the same albedo as Camilla itself (supported by their spectral similarity, see Section 4.3), this magnitude difference implies a size of $12.7 \pm 3.5 \mathrm{~km}$ for S1, smaller than previously reported.

\subsection{Diameter of $\mathrm{S} 2$}

We list in Table A.6 and display in Fig. 12 the 11 measured brightness differences between Camilla and its smaller satellite S2. We measure an average magnitude difference of $\Delta m=9.0 \pm 0.3$ (already reported upon discovery, see Marsset et al., 2016).

Using the diameter of $254 \pm 36 \mathrm{~km}$ for Camilla (Section 5.3) and assuming S2 has the same albedo as Camilla itself as we did for $\mathrm{S} 1$, this magnitude difference implies a size of $4.0 \pm 1.2 \mathrm{~km}$ for S2.

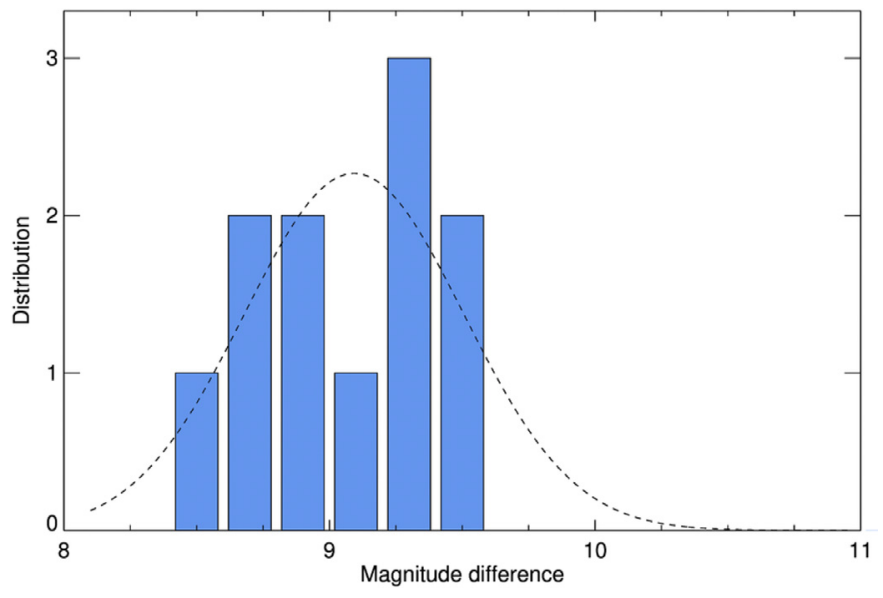

Fig. 12. Distribution of the magnitude differences between Camilla and its second satellite S2. The dashed black line represents the normal distribution fit to our results, with a mean and standard deviation of $9.0 \pm 0.3$.

\section{Discussion}

\subsection{Internal structure}

Using the mass derived from the study of the dynamics of the satellites and the volume from the 3-D shape modeling, we infer a density of $1,280 \pm 130 \mathrm{~kg} \cdot \mathrm{m}^{-3}$ (3 $\sigma$ uncertainty), in agreement with previous reports by Marchis et al. (2008a) and Hanuš et al. (2017). This highlights how critically the density relies on accurate volume estimates: the summary of previous diameter determinations (Table B.2), mainly based on indirect techniques, leads to a density of $1,750 \pm 1,400 \mathrm{~kg} \cdot \mathrm{m}^{-3}$ (3 $\sigma$ uncertainty, Carry, 2012).

The low density found here is comparable to that of (87) Sylvia, a P-type of similar size, also orbiting in the Cybele region (Berthier et al., 2014), and the D-/P-type Jupiter Trojans (617) Patroclus and (624) Hektor (Mueller et al., 2010; Marchis et al., 2014; Buie et al., 2015). As mentioned above (Section 4.2), the most-likely analog material for this type of asteroids are IDPs (Vernazza et al., 2015). There is no measurement of IDP density in the laboratory. However, a density of $3000 \cdot \mathrm{m}^{-3}$ for the silicate phase was reported by the StarDust mission (Brownlee et al., 2006). Because these silicates are mixed with organic carbonaceous particles $\left(\approx 2200 \cdot \mathrm{m}^{-3}\right)$, the density of the bulk material is likely of $\approx 2600 \cdot \mathrm{m}^{-3}$ (Greenberg, 2000; Pätzold et al., 2016). A macroporosity of $50 \pm 9 \%$ would thus be required to explain the density of Camilla, i.e., half of its volume would be occupied by voids. Because the pressure inside Camilla reaches $10^{5}$ Pa less than $15 \mathrm{~km}$ from its surface ( $90 \%$ of the radius), it is unlikely that its structure can sustain such large voids. While silicate grains crush at $10^{7} \mathrm{~Pa}$, larger structures will not resist pressure significantly smaller, as the compressive strength decreases as the power $-1 / 2$ of the size (Lundborg, 1967; Britt et al., 2002).

An alternate explanation to the low density of Camilla may be that it contains large amounts of water ice. An absorption band due to hydration at $3 \mu \mathrm{m}$ was indeed reported by Takir and Emery (2012), whose shape is similar to those of the nearby (24) Themis and (65) Cybele and interpreted as water frost coating on surface grains (Campins et al., 2010; Licandro et al., 2011). Because water ice sublimates on airless surfaces at the heliocentric distance of Themis, Camilla, and Cybele, the ice on the surface must be replenishment from sub-surface reservoir(s) (Rivkin and Emery, 2010), as it occurs on (1) Ceres (A'Hearn and Feldman, 1992; Rousselot et al., 2011; Küppers et al., 2014; Combe et al., 2016). 


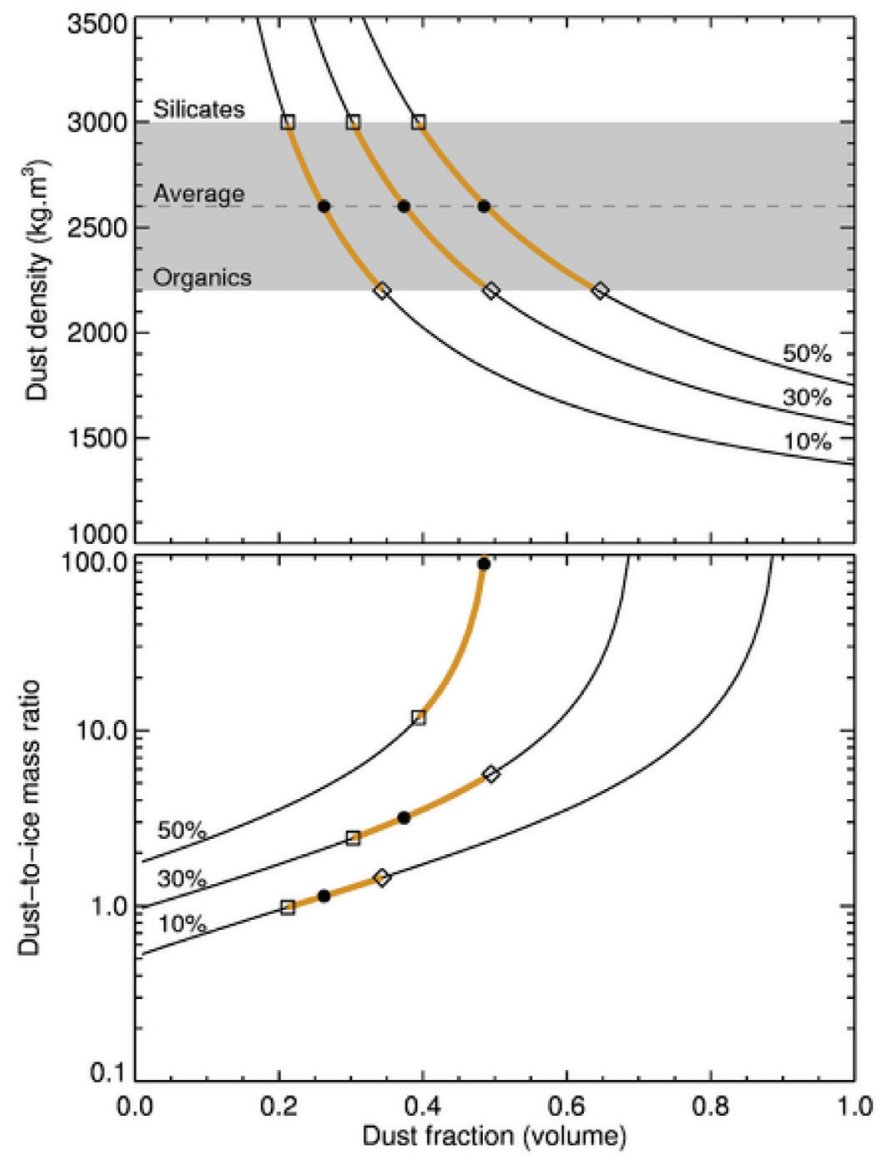

Fig. 13. Top: Dust density as function of its volumetric fraction for different porosities $(10,30,50 \%)$. The expected range from pure organics to pure silicates is represented in shaded gray. Expected range is highlighted in gold. Bottom: Dust-to-ice mass ratios as function of the volumetric fraction of dust.

We thus investigate the possible range of dust-to-ice mass ratios as function of macroporosity in Camilla (Fig. 13). As expected, the porosity decreases with higher ice content and reaches $10-30 \%$ for dust-to-ice mass ratios of 1-6. Therefore, the volume occupied by dust, ice, and voids would be $33 \pm 10 \%, 47 \pm 19 \%$, and $20 \pm 10 \%$ respectively, the latter being preferentially found in the outer-most volume of the asteroid body.

To test this, we compute the gravitational potential quadrupole $J_{2}=0.042 \pm 0.004$ of the 3 -D shape model (Section 5.3) under the assumption of a homogeneous interior using the method of Dobrovolskis (1996). Because the orbit of S1 fits 80 astrometric positions over 15 years to measurement accuracy under the assumption of a null $J_{2}$ (Section 3.3), the mass distribution in Camilla must be more concentrated at the center, with a denser core, than suggested by its shape. Similar internal structure has already been suggested for (87) Sylvia and (624) Hektor by Berthier et al. (2014) and Marchis et al. (2014). Considering a core of pure silicate, and an outer shell of porous ice matching the masses above, the core radius would be $87 \pm 8 \mathrm{~km}$ or $68 \pm 7 \%$ of the radius of Camilla. Additional observations of S2 to determine precisely its orbit are now required to test further the internal structure of Camilla.

\subsection{Future characterization of Camilla triple system}

Owing to the large magnitude difference between Camilla and its satellites (6.5 and 9 mag.), constraining the size and orbit of the satellites by photometric observations of mutual event (eclipses and occultations, see, e.g., Scheirich and Pravec, 2009; Carry et al.,
2015) is not feasible. Observation of S2 will therefore rely on direct imaging such as presented here, or stellar occultations which can moreover provide a direct measurement of the diameter of the satellites. To this effect, we list in Table D.1 a selection of stellar occultations that will occur in the next three years.

Similarly to our work on (87) Sylvia which led to the observation of a stellar occultation by its satellite Romulus (Berthier et al., 2014), we will continuously update the occultation path of Camilla and of its satellites, for these events. The precision of such predictions will benefit from each successive data release of the ESA Gaia astrometry catalogs (Tanga and Delbo, 2007; Gaia Collaboration et al., 2016; Spoto et al., 2017), that will reduce the uncertainty on the path of Camilla itself to a few kilometers. The uncertainty on the occultation path of the satellites will then mostly derive from the uncertainty on their orbital parameters, and we provide them in Table D.1. The orbit of S2 being little constrained, the uncertainty on its position for upcoming occultations is very large. Initial improvement must thus rely on direct imaging of the system.

\section{Summary}

In the present study, we have acquired and compiled optical lightcurves, stellar occultations, visible and near-infrared spectra, and high-contrast and high-angular-resolution images and spectroimages from the Hubble Space Telescope and large ground-based telescopes (Keck, Gemini, VLT) equipped with adaptive-optics-fed cameras.

Using 80 positions spanning 15 years, we study the dynamics of the largest satellite, S1, and determine its orbit around Camilla to be circular, equatorial, and prograde. The residuals between our dynamical solution and the observations are 7.8 mas, corresponding to a sub-pixel accuracy. Using 11 positions of the second, smaller, satellite S2 that we discovered in 2015, we determine a preliminary orbit, marginally inclined from that of S1 and more eccentric. Predictions of the relative position of the satellite with respect to Camilla, critical for planning stellar occultations for instance, are available to the community through our VO service Miriade ${ }^{5}$ (Berthier et al., 2008).

From the visible and near-infrared spectrum of Camilla, we classify it as an Xk-type asteroid, in the Bus-DeMeo taxonomy (DeMeo et al., 2009). Considering its low albedo, it would be classified as a P-type in older taxonomic schemes such as Tedesco's (Tedesco et al., 1989). Using VLT/SPHERE integral-field spectrograph, we measure the near-infrared spectrum of the largest satellite, S1, and compare it with Camilla. No significant differences are found. This, together with its orbital parameters, argue for a formation of the satellite by excavation from impact, re-accumulation of ejecta in orbit, and circularization by tides.

Using optical lightcurves, profiles from disk-resolved imaging, and stellar occultation events, we determine the spin-vector coordinates and 3-D shape of Camilla. The model fits well each data set, and we find a spherical-volume-equivalent diameter of $254 \pm 36 \mathrm{~km}$. By combining the mass from the dynamics with the volume of the shape model, we find a density of $1,280 \pm 130 \mathrm{~kg} \cdot \mathrm{m}^{-3}$. Considering Camilla's most likely analog material are IDPs, this implies a macroporosity of $50 \pm 9 \%$, likely too high to be sustained. By considering a mixture of ice and silicate, the macroporosity could be in the range $10-30 \%$ for a dust-to-ice mass ratio of 1-6, the denser material being concentrated toward the center as suggested by the dynamics of the system.

\footnotetext{
5 http://vo.imcce.fr/
} 


\section{Acknowledgments}

Based on observations collected at the European Organisation for Astronomical Research in the Southern Hemisphere under ESO programmes 071.C-0669 (PI Merline), 073.C-0062 \& 074.C-0052 (PI Marchis), 088.C-0528 (PI Rojo), 095.C-0217 \& 297.C-5034 (PI Marsset).

Some of the data presented herein were obtained at the W.M. Keck Observatory, which is operated as a scientific partnership among the California Institute of Technology, the University of California and the National Aeronautics and Space Administration. The Observatory was made possible by the generous financial support of the W.M. Keck Foundation.

This research has made use of the Keck Observatory Archive (KOA), which is operated by the W. M. Keck Observatory and the NASA Exoplanet Science Institute (NExScI), under contract with the National Aeronautics and Space Administration.

Some of these observations were acquired under grants from the National Science Foundation and NASA to Merline (PI).

The authors wish to recognize and acknowledge the very significant cultural role and reverence that the summit of Mauna Kea has always had within the indigenous Hawaiian community. We are most fortunate to have the opportunity to conduct observations from this mountain.

Based on observations obtained at the Gemini Observatory, which is operated by the Association of Universities for Research in Astronomy, Inc., under a cooperative agreement with the NSF on behalf of the Gemini partnership: the National Science Founda- tion (United States), the National Research Council (Canada), CONICYT (Chile), Ministerio de Ciencia, Tecnología e Innovación Productiva (Argentina), and Ministério da Ciência, Tecnologia e Inovação (Brazil).

We wish to acknowledge the support of NASA Contract NAS526555 and STScI grant GO-05583.01 to Alex Storrs (PI).

Visiting Astronomer at the Infrared Telescope Facility, which is operated by the University of Hawaii under contract NNH14CK55B with the National Aeronautics and Space Administration.

We thank the AGORA association which administrates the $60 \mathrm{~cm}$ telescope at Les Makes observatory, under a financial agreement with Paris Observatory. Thanks to A. Peyrot, J.-P. Teng for local support, and A. Klotz for helping with the robotizing.

Thanks to all the amateurs worldwide who regularly observe asteroid lightcurves and stellar occultations. Many co-authors of this study are amateurs who observed Camilla, and provided crucial data.

We thank J. Ďurech for providing his implementation of Dobrovolskis (1996) method.

The authors acknowledge the use of the Virtual Observatory tools Miriade ${ }^{6}$ (Berthier et al., 2008), $\mathrm{MP}^{3} \mathrm{C}^{7}$ (Delbo et al., 2017), TOPCAT $^{8}$, and STILTS ${ }^{9}$ (Taylor, 2005). This research used the facilities of the Canadian Astronomy Data Centre operated by the National Research Council of Canada with the support of the Canadian Space Agency (Gwyn et al., 2012).

\footnotetext{
${ }^{6}$ http://vo.imcce.fr/

7 https://mp3c.oca.eu

8 http://www.star.bristol.ac.uk/-pl2X-sim-mbt/topcat/

9 http://www.star.bristol.ac.uk/-pl2X-sim-mbt/stilts/
} 


\section{Appendix A. Details on the observing data sets}

We provide here the details for each lightcurve (Table A.1), disk-resolved image (Table A.2), and stellar occultation (Table A.4), as well as the astrometry and photometry of S1 (Table A.5) and S2 (Table A.6).

Table A.1

Date, duration $\left(\mathcal{L}\right.$, in hours), number of points $\left(\mathcal{N}_{p}\right)$, phase angle $(\alpha)$, filter, residual (against the shape model), IAU code, and observers, for each lightcurve.

\begin{tabular}{|c|c|c|c|c|c|c|c|c|}
\hline & Date & $\begin{array}{l}\mathcal{L} \\
\text { (h) }\end{array}$ & $\mathcal{N}_{p}$ & $\begin{array}{l}\alpha \\
\left(^{\circ}\right)\end{array}$ & Filter & $\begin{array}{l}\text { RMS } \\
\text { (mag) }\end{array}$ & IAU & Observers \\
\hline 1 & 1981-02-01 & 4.0 & 5 & 2.9 & V & 0.012 & 654 & Harris and Young (1989) \\
\hline 2 & 1981-02-02 & 6.2 & 9 & 2.8 & V & 0.023 & 654 & Harris and Young (1989) \\
\hline 3 & $1981-02-04$ & 7.7 & 10 & 2.7 & $\mathrm{~V}$ & 0.018 & 654 & Harris and Young (1989) \\
\hline 4 & $1981-02-05$ & 5.6 & 14 & 2.8 & V & 0.016 & 654 & Harris and Young (1989) \\
\hline 5 & 1982-01-09 & 2.4 & 11 & 16.4 & V & 0.021 & 695 & Weidenschilling et al. (1987) \\
\hline 6 & $1982-01-15$ & 4.4 & 8 & 16.6 & $\mathrm{~V}$ & 0.047 & 695 & Weidenschilling et al. (1987) \\
\hline 7 & $1982-05-20$ & 4.5 & 19 & 10.6 & $\mathrm{~V}$ & 0.028 & 695 & Weidenschilling et al. (1987) \\
\hline 8 & $1982-06-23$ & 4.6 & 6 & 15.8 & V & 0.030 & 695 & Weidenschilling et al. (1987) \\
\hline 9 & $1982-06-24$ & 2.1 & 8 & 15.9 & V & 0.037 & 695 & Weidenschilling et al. (1987) \\
\hline 10 & $1982-06-25$ & 2.9 & 15 & 16.0 & $\mathrm{~V}$ & 0.020 & 695 & Weidenschilling et al. (1987) \\
\hline 11 & $1983-03-27$ & 2.0 & 10 & 15.5 & $\mathrm{~V}$ & 0.048 & 695 & Weidenschilling et al. (1987) \\
\hline 12 & 1983-03-29 & 4.3 & 5 & 15.4 & V & 0.070 & 695 & Weidenschilling et al. (1987) \\
\hline 13 & $1983-05-24$ & 4.8 & 35 & 7.6 & V & 0.027 & 695 & Weidenschilling et al. (1987) \\
\hline 14 & 1983-07-03 & 4.7 & 23 & 6.1 & $\mathrm{~V}$ & 0.021 & 695 & Weidenschilling et al. (1987) \\
\hline 15 & 1984-06-07 & 2.2 & 11 & 14.8 & V & 0.026 & 695 & Weidenschilling et al. (1987) \\
\hline 16 & 1984-06-10 & 4.6 & 10 & 14.5 & V & 0.019 & 695 & Weidenschilling et al. (1987) \\
\hline 17 & 1984-07-05 & 3.0 & 15 & 10.7 & $\mathrm{~V}$ & 0.047 & 695 & Weidenschilling et al. (1987) \\
\hline 18 & 1984-08-16 & 5.5 & 32 & 2.3 & V & 0.024 & 809 & di Martino et al. (1987) \\
\hline 19 & $1985-10-20$ & 4.6 & 21 & 3.0 & V & 0.019 & 695 & Weidenschilling et al. (1987) \\
\hline 20 & 1987-02-06 & 2.7 & 20 & 13.6 & V & 0.018 & 695 & Weidenschilling et al. (1990) \\
\hline 21 & 1987-02-07 & 4.7 & 17 & 13.8 & $\mathrm{~V}$ & 0.019 & 695 & Weidenschilling et al. (1990) \\
\hline 22 & $1988-04-25$ & 4.7 & 15 & 13.1 & V & 0.023 & 695 & Weidenschilling et al. (1990) \\
\hline 23 & $1988-04-26$ & 3.6 & 20 & 13.3 & V & 0.027 & 695 & Weidenschilling et al. (1990) \\
\hline 24 & 1988-04-29 & 2.9 & 16 & 13.9 & $\mathrm{~V}$ & 0.032 & 695 & Weidenschilling et al. (1990) \\
\hline 25 & 2004-09-19 & 6.8 & 15 & 2.0 & C & 0.013 & A14 & L. Bernasconi \\
\hline 26 & 2004-11-05 & 5.6 & 37 & 13.7 & $\mathrm{C}$ & 0.025 & A14 & L. Bernasconi \\
\hline 27 & 2008-03-07 & 2.0 & 20 & 11.5 & clear & 0.060 & 950 & SuperWASP - J. Grice \\
\hline 28 & 2008-03-13 & 5.2 & 76 & 10.7 & clear & 0.029 & 950 & SuperWASP - J. Grice \\
\hline 29 & $2008-03-17$ & 3.0 & 53 & 9.4 & clear & 0.028 & 950 & SuperWASP - J. Grice \\
\hline 30 & 2008-03-18 & 5.3 & 105 & 8.7 & clear & 0.036 & 950 & SuperWASP - J. Grice \\
\hline 31 & 2008-03-29 & 3.3 & 29 & 5.5 & clear & 0.028 & 950 & SuperWASP - J. Grice \\
\hline 32 & $2008-03-29$ & 4.0 & 40 & 5.5 & clear & 0.033 & 950 & SuperWASP - J. Grice \\
\hline 33 & $2008-03-30$ & 4.5 & 22 & 5.2 & clear & 0.021 & 950 & SuperWASP - J. Grice \\
\hline 34 & 2008-04-01 & 5.0 & 61 & 4.6 & clear & 0.025 & 950 & SuperWASP - J. Grice \\
\hline 35 & $2008-04-13$ & 4.8 & 69 & 2.2 & clear & 0.041 & 950 & SuperWASP - J. Grice \\
\hline 36 & 2008-04-13 & 0.7 & 14 & 2.2 & clear & 0.011 & 950 & SuperWASP - J. Grice \\
\hline 37 & 2008-04-16 & 4.9 & 65 & 2.2 & clear & 0.033 & 950 & SuperWASP - J. Grice \\
\hline 38 & 2008-04-16 & 4.1 & 128 & 2.4 & clear & 0.027 & 950 & SuperWASP - J. Grice \\
\hline 39 & $2008-04-22$ & 4.2 & 58 & 3.7 & clear & 0.040 & 950 & SuperWASP - J. Grice \\
\hline 40 & $2008-04-22$ & 5.3 & 77 & 3.7 & clear & 0.029 & 950 & SuperWASP - J. Grice \\
\hline 41 & $2008-04-23$ & 3.9 & 44 & 4.0 & clear & 0.030 & 950 & SuperWASP - J. Grice \\
\hline 42 & $2008-04-23$ & 3.3 & 36 & 4.0 & clear & 0.023 & 950 & SuperWASP - J. Grice \\
\hline 43 & $2008-04-24$ & 3.2 & 44 & 4.2 & clear & 0.019 & 950 & SuperWASP - J. Grice \\
\hline 44 & $2008-04-24$ & 3.7 & 51 & 4.2 & clear & 0.020 & 950 & SuperWASP - J. Grice \\
\hline 45 & $2008-04-27$ & 2.4 & 32 & 5.1 & clear & 0.021 & 950 & SuperWASP - J. Grice \\
\hline 46 & $2008-04-28$ & 3.3 & 52 & 5.4 & clear & 0.033 & 950 & SuperWASP - J. Grice \\
\hline 47 & 2008-04-29 & 5.2 & 61 & 5.7 & clear & 0.029 & 950 & SuperWASP - J. Grice \\
\hline 48 & $2008-05-04$ & 1.4 & 19 & 7.1 & clear & 0.025 & 950 & SuperWASP - J. Grice \\
\hline 49 & 2008-05-09 & 3.8 & 47 & 7.7 & clear & 0.028 & 950 & SuperWASP - J. Grice \\
\hline 50 & $2008-05-10$ & 3.2 & 93 & 8.5 & clear & 0.026 & 950 & SuperWASP - J. Grice \\
\hline 51 & $2008-05-10$ & 4.6 & 109 & 8.8 & clear & 0.028 & 950 & SuperWASP - J. Grice \\
\hline 52 & 2008-05-12 & 3.6 & 66 & 9.0 & clear & 0.022 & 950 & SuperWASP - J. Grice \\
\hline 53 & $2008-05-12$ & 3.9 & 81 & 9.3 & clear & 0.048 & 950 & SuperWASP - J. Grice \\
\hline 54 & 2008-05-13 & 4.5 & 67 & 9.6 & clear & 0.036 & 950 & SuperWASP - J. Grice \\
\hline 55 & 2008-05-19 & 2.4 & 119 & 10.8 & clear & 0.048 & 950 & SuperWASP - J. Grice \\
\hline 56 & $2008-05-20$ & 3.8 & 69 & 11.3 & clear & 0.048 & 950 & SuperWASP - J. Grice \\
\hline 57 & $2008-05-31$ & 1.5 & 44 & 13.5 & $\mathrm{C}$ & 0.030 & 181 & Polishook (2009) \\
\hline 58 & 2008-06-05 & 1.1 & 13 & 14.3 & clear & 0.043 & 950 & SuperWASP - J. Grice \\
\hline 59 & 2008-06-06 & 4.5 & 19 & 14.7 & clear & 0.032 & 950 & SuperWASP - J. Grice \\
\hline 60 & 2008-06-06 & 1.2 & 20 & 14.9 & clear & 0.049 & 950 & SuperWASP - J. Grice \\
\hline 61 & $2008-06-10$ & 4.8 & 83 & 15.0 & clear & 0.044 & 950 & SuperWASP - J. Grice \\
\hline 62 & 2008-06-10 & 2.4 & 46 & 16.3 & clear & 0.039 & 950 & SuperWASP - J. Grice \\
\hline
\end{tabular}


Table A.1 (continued)

\begin{tabular}{|c|c|c|c|c|c|c|c|c|}
\hline & Date & $\begin{array}{l}\mathcal{L} \\
(\mathrm{h})\end{array}$ & $\mathcal{N}_{p}$ & $\begin{array}{l}\alpha \\
\left({ }^{\circ}\right)\end{array}$ & Filter & $\begin{array}{l}\text { RMS } \\
\text { (mag) }\end{array}$ & IAU & Observers \\
\hline 63 & $2008-06-23$ & 4.2 & 83 & 16.4 & clear & 0.036 & 950 & SuperWASP - J. Grice \\
\hline 64 & $2008-06-24$ & 4.1 & 81 & 16.5 & clear & 0.032 & 950 & SuperWASP - J. Grice \\
\hline 65 & $2008-06-27$ & 1.7 & 63 & 16.6 & $\mathrm{C}$ & 0.019 & 181 & Polishook (2009) \\
\hline 66 & $2008-06-28$ & 2.0 & 82 & 16.6 & $\mathrm{C}$ & 0.018 & 181 & Polishook (2009) \\
\hline 67 & 2010-07-09 & 2.7 & 86 & 10.7 & $\mathrm{C}$ & 0.019 & 615 & J. Montier \& S. Heterier \\
\hline 68 & $2010-07-10$ & 3.3 & 89 & 10.5 & C & 0.019 & 517 & F. Reignier \\
\hline 69 & 2010-07-10 & 3.9 & 57 & 10.5 & clear & 0.063 & 950 & SuperWASP - J. Grice \\
\hline 70 & 2010-07-11 & 3.1 & 140 & 10.3 & $\mathrm{C}$ & 0.020 & 615 & J. Montier \& S. Heterier \\
\hline 71 & 2010-07-11 & 4.1 & 85 & 10.2 & clear & 0.024 & 950 & SuperWASP - J. Grice \\
\hline 72 & 2010-07-11 & 4.1 & 86 & 10.0 & clear & 0.031 & 950 & SuperWASP - J. Grice \\
\hline 73 & 2010-07-13 & 4.0 & 87 & 9.8 & clear & 0.087 & 950 & SuperWASP - J. Grice \\
\hline 74 & 2010-07-14 & 4.3 & 93 & 9.6 & clear & 0.040 & 950 & SuperWASP - J. Grice \\
\hline 75 & 2010-07-14 & 4.4 & 96 & 9.4 & clear & 0.143 & 950 & SuperWASP - J. Grice \\
\hline 76 & 2010-07-16 & 3.3 & 91 & 8.9 & clear & 0.028 & 950 & SuperWASP - J. Grice \\
\hline 77 & 2010-07-18 & 2.7 & 91 & 8.4 & clear & 0.036 & 950 & SuperWASP - J. Grice \\
\hline 78 & 2010-07-19 & 3.3 & 106 & 8.2 & clear & 0.043 & 950 & SuperWASP - J. Grice \\
\hline 79 & $2010-07-20$ & 3.1 & 104 & 7.9 & clear & 0.035 & 950 & SuperWASP - J. Grice \\
\hline 80 & $2010-07-23$ & 2.4 & 54 & 7.4 & clear & 0.035 & 950 & SuperWASP - J. Grice \\
\hline 81 & $2010-07-23$ & 5.0 & 97 & 7.2 & clear & 0.052 & 950 & SuperWASP - J. Grice \\
\hline 82 & 2010-08-01 & 5.3 & 112 & 4.8 & clear & 0.037 & 950 & SuperWASP - J. Grice \\
\hline 83 & 2010-08-03 & 2.7 & 47 & 4.3 & clear & 0.035 & 950 & SuperWASP - J. Grice \\
\hline 84 & $2010-08-30$ & 5.1 & 109 & 4.7 & clear & 0.038 & 950 & SuperWASP - J. Grice \\
\hline 85 & $2010-08-31$ & 5.3 & 114 & 5.0 & clear & 0.029 & 950 & SuperWASP - J. Grice \\
\hline 86 & 2010-09-02 & 1.6 & 22 & 5.5 & clear & 0.023 & 950 & SuperWASP - J. Grice \\
\hline 87 & 2010-09-03 & 5.2 & 111 & 5.8 & clear & 0.030 & 950 & SuperWASP - J. Grice \\
\hline 88 & 2010-09-04 & 5.1 & 111 & 6.0 & clear & 0.030 & 950 & SuperWASP - J. Grice \\
\hline 89 & 2010-09-05 & 3.4 & 71 & 6.3 & clear & 0.070 & 950 & SuperWASP - J. Grice \\
\hline 90 & 2010-09-08 & 5.2 & 73 & 7.1 & clear & 0.029 & 950 & SuperWASP - J. Grice \\
\hline 91 & 2010-09-08 & 0.9 & 32 & 7.3 & clear & 0.031 & 950 & SuperWASP - J. Grice \\
\hline 92 & 2010-09-09 & 1.8 & 23 & 7.6 & clear & 0.024 & 950 & SuperWASP - J. Grice \\
\hline 93 & 2010-09-11 & 5.1 & 103 & 7.8 & clear & 0.033 & 950 & SuperWASP - J. Grice \\
\hline 94 & 2010-09-30 & 1.9 & 54 & 12.0 & clear & 0.042 & 950 & SuperWASP - J. Grice \\
\hline 95 & 2010-10-01 & 4.0 & 84 & 12.1 & clear & 0.052 & 950 & SuperWASP - J. Grice \\
\hline 96 & $2015-04-20$ & 3.6 & 70 & 8.2 & $\mathrm{R}$ & 0.028 & 181 & F. Vachier \\
\hline 97 & $2015-04-21$ & 5.7 & 108 & 7.9 & $\mathrm{R}$ & 0.027 & 181 & F. Vachier \\
\hline 98 & $2015-04-23$ & 5.5 & 87 & 7.4 & $\mathrm{R}$ & 0.025 & 181 & F. Vachier \\
\hline 99 & $2015-04-24$ & 6.6 & 118 & 7.2 & $\mathrm{R}$ & 0.023 & 181 & F. Vachier \\
\hline 100 & 2015-05-09 & 1.4 & 24 & 3.9 & $\mathrm{R}$ & 0.024 & 181 & F. Vachier \\
\hline 101 & 2015-05-11 & 4.9 & 84 & 3.6 & $\mathrm{R}$ & 0.021 & 181 & F. Vachier \\
\hline 102 & 2015-05-12 & 5.8 & 44 & 3.6 & $\mathrm{R}$ & 0.029 & 181 & F. Vachier \\
\hline 103 & 2015-05-13 & 5.2 & 85 & 3.5 & $\mathrm{R}$ & 0.022 & 181 & F. Vachier \\
\hline 104 & 2015-05-17 & 3.8 & 58 & 3.5 & $\mathrm{R}$ & 0.025 & 181 & F. Vachier \\
\hline 105 & 2015-05-18 & 5.8 & 89 & 3.6 & $\mathrm{R}$ & 0.018 & 181 & F. Vachier \\
\hline 106 & 2015-05-19 & 5.1 & 61 & 3.7 & $\mathrm{R}$ & 0.024 & 181 & F. Vachier \\
\hline 107 & $2015-05-20$ & 6.0 & 91 & 3.8 & $\mathrm{R}$ & 0.021 & 181 & F. Vachier \\
\hline 108 & $2015-05-21$ & 5.8 & 106 & 4.0 & $\mathrm{R}$ & 0.021 & 181 & F. Vachier \\
\hline 109 & $2015-05-22$ & 5.4 & 98 & 4.1 & $\mathrm{R}$ & 0.021 & 181 & F. Vachier \\
\hline 110 & $2015-05-23$ & 6.3 & 102 & 4.3 & $\mathrm{R}$ & 0.021 & 181 & F. Vachier \\
\hline 111 & $2015-05-24$ & 1.0 & 14 & 4.5 & $\mathrm{R}$ & 0.024 & 181 & F. Vachier \\
\hline 112 & $2015-05-26$ & 1.9 & 36 & 4.9 & $\mathrm{R}$ & 0.021 & 181 & F. Vachier \\
\hline 113 & 2015-06-03 & 3.6 & 68 & 6.8 & $\mathrm{R}$ & 0.022 & 181 & F. Vachier \\
\hline 114 & 2015-06-03 & 5.5 & 251 & 6.8 & V & 0.026 & 517 & D. Romeuf \\
\hline 115 & $2015-06-04$ & 4.2 & 76 & 7.0 & $\mathrm{R}$ & 0.025 & 181 & F. Vachier \\
\hline 116 & 2015-06-05 & 5.0 & 75 & 7.3 & $\mathrm{R}$ & 0.019 & 181 & F. Vachier \\
\hline 117 & 2015-06-05 & 4.9 & 274 & 7.3 & V & 0.026 & 517 & D. Romeuf \\
\hline 118 & 2015-06-09 & 3.2 & 59 & 8.3 & $\mathrm{R}$ & 0.024 & 181 & F. Vachier \\
\hline 119 & 2015-06-10 & 3.0 & 38 & 8.5 & $\mathrm{R}$ & 0.021 & 181 & F. Vachier \\
\hline 120 & $2015-06-11$ & 1.4 & 27 & 8.7 & $\mathrm{R}$ & 0.024 & 181 & F. Vachier \\
\hline 121 & 2015-06-17 & 5.4 & 98 & 10.1 & $\mathrm{R}$ & 0.024 & 181 & F. Vachier \\
\hline 122 & $2015-06-20$ & 28.2 & 376 & 10.7 & $\mathrm{R}$ & 0.023 & 586 & S. Fauvaud \\
\hline 123 & $2015-06-22$ & 5.8 & 104 & 11.2 & $\mathrm{R}$ & 0.052 & 181 & F. Vachier \\
\hline 124 & 2015-06-23 & 2.2 & 40 & 11.4 & $\mathrm{R}$ & 0.036 & 181 & F. Vachier \\
\hline 125 & $2015-06-25$ & 4.7 & 88 & 11.8 & $\mathrm{R}$ & 0.026 & 181 & F. Vachier \\
\hline 126 & $2015-06-26$ & 3.8 & 70 & 12.0 & $\mathrm{R}$ & 0.029 & 181 & F. Vachier \\
\hline 127 & 2015-07-06 & 3.8 & 71 & 13.7 & $\mathrm{R}$ & 0.029 & 181 & F. Vachier \\
\hline
\end{tabular}


Table A.2

Date, mid-observing time (UTC), heliocentric distance $(\Delta)$ and range to observer $(r)$, phase angle $(\alpha)$, apparent size $(\Theta)$, longitude $(\lambda)$ and latitude $(\beta)$ of the subsolar and subobserver points (SSP, SEP).

\begin{tabular}{|c|c|c|c|c|c|c|c|c|c|c|}
\hline & Date & UTC & $\begin{array}{l}\Delta \\
(\mathrm{AU})\end{array}$ & $\begin{array}{l}r \\
(\mathrm{AU})\end{array}$ & $\begin{array}{l}\alpha \\
\left(^{\circ}\right)\end{array}$ & $\begin{array}{l}\Theta \\
\left({ }^{\prime \prime}\right)\end{array}$ & $\begin{array}{l}\mathrm{SEP}_{\lambda} \\
\left(^{\circ}\right)\end{array}$ & $\begin{array}{l}\operatorname{SEP}_{\beta} \\
\left(^{\circ}\right)\end{array}$ & $\begin{array}{l}\operatorname{SSP}_{\lambda} \\
\left(^{\circ}\right)\end{array}$ & $\begin{array}{l}\operatorname{SSP}_{\beta} \\
\left(^{\circ}\right)\end{array}$ \\
\hline 1 & 2003-08-15 & $08: 35: 22$ & 3.75 & 2.87 & 8.5 & 0.119 & 46.0 & 12.8 & 54.7 & 12.0 \\
\hline 2 & 2003-08-17 & 10:50:07 & 3.75 & 2.88 & 9.0 & 0.117 & 271.7 & 13.0 & 280.9 & 12.0 \\
\hline 3 & 2009-06-07 & $11: 25: 55$ & 3.68 & 2.71 & 5.0 & 0.124 & 267.1 & 15.9 & 264.3 & 20.1 \\
\hline 4 & $2010-06-28$ & $10: 19: 28$ & 3.74 & 3.04 & 12.6 & 0.116 & 231.4 & -1.9 & 221.2 & 5.7 \\
\hline 5 & 2004-09-01 & $05: 17: 22$ & 3.66 & 2.67 & 3.7 & 0.118 & 120.4 & -8.5 & 117.3 & -6.3 \\
\hline 6 & 2004-09-08 & $06: 41: 20$ & 3.65 & 2.65 & 1.5 & 0.119 & 129.5 & -7.6 & 128.3 & -6.7 \\
\hline 7 & 2004-09-13 & $03: 42: 51$ & 3.65 & 2.65 & 0.2 & 0.142 & 71.4 & -7.0 & 71.6 & -6.9 \\
\hline 8 & 2004-09-13 & $05: 47: 28$ & 3.65 & 2.65 & 0.2 & 0.126 & 277.1 & -7.0 & 277.2 & -6.9 \\
\hline 9 & 2004-09-14 & 04:09:30 & 3.65 & 2.65 & 0.4 & 0.138 & 54.6 & -6.9 & 55.0 & -7.0 \\
\hline 10 & 2004-09-14 & 07:06:44 & 3.65 & 2.65 & 0.5 & 0.139 & 195.0 & -6.9 & 195.5 & -7.0 \\
\hline 11 & 2004-09-14 & $07: 14: 31$ & 3.65 & 2.65 & 0.5 & 0.133 & 185.4 & -6.9 & 185.9 & -7.0 \\
\hline 12 & 2004-09-15 & $04: 18: 34$ & 3.65 & 2.65 & 0.7 & 0.140 & 59.6 & -6.8 & 60.3 & -7.0 \\
\hline 13 & 2004-09-15 & $04: 26: 55$ & 3.65 & 2.65 & 0.7 & 0.134 & 49.2 & -6.8 & 49.9 & -7.0 \\
\hline 14 & 2004-09-16 & $04: 48: 18$ & 3.65 & 2.65 & 1.1 & 0.134 & 38.9 & -6.6 & 39.9 & -7.1 \\
\hline 15 & 2004-10-07 & $02: 12: 52$ & 3.64 & 2.72 & 7.4 & 0.141 & 212.4 & -4.3 & 218.7 & -8.1 \\
\hline 16 & 2004-10-08 & $02: 22: 37$ & 3.64 & 2.73 & 7.6 & 0.136 & 216.6 & -4.2 & 223.2 & -8.1 \\
\hline 17 & 2011-11-08 & $03: 21: 35$ & 3.50 & 2.59 & 7.7 & 0.131 & 283.0 & -13.0 & 289.4 & -17.7 \\
\hline 18 & $2011-11-10$ & 01:22:04 & 3.50 & 2.61 & 8.2 & 0.134 & 103.6 & -12.9 & 110.5 & -17.8 \\
\hline 19 & $2015-05-29$ & $04: 38: 45$ & 3.58 & 2.61 & 5.4 & 0.120 & 350.8 & 18.1 & 354.8 & 22.0 \\
\hline 20 & $2015-05-29$ & $04: 51: 26$ & 3.58 & 2.61 & 5.4 & 0.118 & 335.1 & 18.1 & 339.1 & 22.0 \\
\hline 21 & 2015-05-29 & $05: 07: 36$ & 3.58 & 2.61 & 5.5 & 0.120 & 315.0 & 18.1 & 319.1 & 22.0 \\
\hline 22 & $2015-05-29$ & $05: 15: 12$ & 3.58 & 2.61 & 5.5 & 0.122 & 305.6 & 18.1 & 309.6 & 22.0 \\
\hline 23 & 2015-05-29 & $05: 25: 54$ & 3.58 & 2.61 & 5.5 & 0.125 & 292.4 & 18.1 & 296.4 & 22.0 \\
\hline 24 & $2015-05-29$ & $05: 28: 58$ & 3.58 & 2.61 & 5.5 & 0.126 & 288.6 & 18.1 & 292.6 & 22.0 \\
\hline 25 & $2015-05-29$ & $05: 32: 04$ & 3.58 & 2.61 & 5.5 & 0.127 & 284.7 & 18.1 & 288.8 & 22.0 \\
\hline 26 & 2016-07-12 & 05:06:10 & 3.72 & 2.72 & 3.4 & 0.140 & 233.1 & 10.4 & 233.3 & 13.8 \\
\hline 27 & $2016-07-12$ & $05: 13: 32$ & 3.72 & 2.72 & 3.4 & 0.142 & 224.0 & 10.4 & 224.1 & 13.8 \\
\hline 28 & 2016-07-12 & $05: 20: 55$ & 3.72 & 2.72 & 3.4 & 0.137 & 214.8 & 10.4 & 215.0 & 13.8 \\
\hline 29 & 2016-07-28 & $05: 52: 47$ & 3.72 & 2.74 & 4.8 & 0.139 & 74.3 & 11.8 & 79.0 & 13.2 \\
\hline 30 & $2016-07-28$ & 05:59:03 & 3.72 & 2.74 & 4.8 & 0.139 & 66.5 & 11.8 & 71.3 & 13.2 \\
\hline 31 & $2016-07-28$ & $06: 05: 21$ & 3.72 & 2.74 & 4.8 & 0.140 & 58.7 & 11.8 & 63.5 & 13.2 \\
\hline 32 & 2016-07-30 & 01:39:02 & 3.72 & 2.75 & 5.2 & 0.128 & 61.0 & 11.9 & 66.3 & 13.1 \\
\hline 33 & $2016-07-30$ & $01: 46: 07$ & 3.72 & 2.75 & 5.2 & 0.129 & 52.3 & 11.9 & 57.5 & 13.1 \\
\hline 34 & 2016-07-30 & 01:53:12 & 3.72 & 2.75 & 5.2 & 0.130 & 43.5 & 11.9 & 48.7 & 13.1 \\
\hline
\end{tabular}

Table A.3

Timing and location of each observer for the stellar occultations used in this work.

\begin{tabular}{|c|c|c|c|c|c|}
\hline Observer & Location & $\begin{array}{l}\text { Latitude } \\
\left({ }^{\circ}\right)\end{array}$ & $\begin{array}{l}\text { Longitude } \\
\left({ }^{\circ}\right)\end{array}$ & $\begin{array}{l}\text { Disappearance } \\
\text { (UT) }\end{array}$ & $\begin{array}{l}\text { Reappearance } \\
\text { (UT) }\end{array}$ \\
\hline \multicolumn{6}{|c|}{2004 September 5} \\
\hline Randy Peterson & Cave Creek Desert, AZ & 33.813 & -112.0002 & $8: 54: 49.1$ & $8: 55: 03.9$ \\
\hline Paul Maley/Syd Leach & Fountain Hills, AZ & 33.6278 & -111.8333 & $8: 54: 50.96$ & $8: 55: 02.70$ \\
\hline Scott Donnell & Eastonville, CO & 39.0725 & -104.5778 & $8: 54: 01.5$ & $8: 54: 12.0$ \\
\hline \multicolumn{6}{|c|}{2010 September 16} \\
\hline Kerry Coughlin & LaPaz, Baja, Mexico & 24.1387 & -110.3296 & Miss & Miss \\
\hline \multicolumn{6}{|c|}{2015 January 01} \\
\hline Andy Scheck & Scaggsville, MD & 40.3511 & -77.0 & $11: 26: 31.76$ & $11: 26: 34.77$ \\
\hline Bob Dunford & Naperville, IL & 41.759 & -88.1167 & Miss & Miss \\
\hline Chad Ellington & Owings, MD & 38.6906 & -76.6354 & Miss & Miss \\
\hline \multicolumn{6}{|c|}{2015 February 12} \\
\hline Derek Breit & Morgan Hill, CA & 37.1133 & -121.7028 & $11: 58: 13.97$ & $11: 58: 23.98$ \\
\hline Derek Breit (double star) & Morgan Hill, CA & 37.1133 & -121.7028 & $11: 58: 11.03$ & $11: 58: 25.48$ \\
\hline Ted Blank & Payson, AZ & 34.2257 & -111.2988 & $11: 58: 56.82$ & $11: 58: 58.55$ \\
\hline Chuck McPartlin & Santa Barbara, CA & 34.4567 & -119.795 & Miss & Miss \\
\hline Tony George & Scottsdale, AZ & 33.7157 & -111.8494 & Miss & Miss \\
\hline Sam Herchak & Mesa, AZ & 33.3967 & -111.6985 & Miss & Miss \\
\hline
\end{tabular}


Table A.3 (continued)

\begin{tabular}{|c|c|c|c|c|c|}
\hline Observer & Location & $\begin{array}{l}\text { Latitude } \\
\left({ }^{\circ}\right)\end{array}$ & $\begin{array}{l}\text { Longitude } \\
\left({ }^{\circ}\right)\end{array}$ & $\begin{array}{l}\text { Disappearance } \\
\text { (UT) }\end{array}$ & $\begin{array}{l}\text { Reappearance } \\
\text { (UT) }\end{array}$ \\
\hline \multicolumn{6}{|c|}{2015 May 06} \\
\hline Dan Caton & Boone, NC & 36.2514 & -81.4122 & $5: 23: 07.46$ & $5: 23: 19.46$ \\
\hline Steve Messner & Bevier, MO & 39.7722 & -92.5243 & $5: 24: 02.71$ & $5: 24: 20.96$ \\
\hline Roger Venable & Elgin, SC & 34.1476 & -80.7502 & $5: 22: 54.75$ & $5: 23: 09.05$ \\
\hline Roger Venable & New Holland, SC & 33.7397 & -81.5201 & Miss & Miss \\
\hline Roger Venable & Hepzibah, GA & 33.3394 & -82.1542 & Miss & Miss \\
\hline Chris Anderson & Twin Falls, ID & 42.5839 & -114.4703 & Miss & Miss \\
\hline Chuck McPartlin & Santa Barbara, CA & 34.4568 & -119.7951 & Miss & Miss \\
\hline \multicolumn{6}{|c|}{2015 August 23} \\
\hline Steve Preston & Carnation, WA & 47.6437 & -121.9224 & $4: 17: 35.07$ & 4:17:42.89 \\
\hline Andrea Dobson/Larry North & Walla Walla, WA & 46.0044 & -118.8928 & 4:17:49.06 & 4:17:59.13 \\
\hline Tony George & Umatilla, OR & 45.9221 & -119.2983 & $4: 17: 47.72$ & $4: 17: 58.54$ \\
\hline Chad Ellington & Tumwater, WA & 46.9763 & -122.9111 & $4: 17: 31.85$ & $4: 17: 44.46$ \\
\hline Chris Anderson & Twin Falls, ID & 42.5839 & -114.4703 & 4:18:14.41 & $4: 18: 27.46$ \\
\hline David Becker & Grasmere, ID & 42.6733 & -115.8981 & 4:18:10.17 & $4: 18: 23.25$ \\
\hline William Gimple & Greenville, CA & 40.1377 & -120.8667 & Miss & Miss \\
\hline Charles Arrowsmith & Quincy, CA & 39.9477 & -120.9691 & Miss & Miss \\
\hline Tom Beard & Reno, NV & 39.3729 & -119.8312 & Miss & Miss \\
\hline Jerry Bardecker & Gardnerville, NV & 38.8899 & -119.6723 & Miss & Miss \\
\hline Ted Swift & Davis, CA & 38.5522 & -121.7856 & Miss & Miss \\
\hline \multicolumn{6}{|c|}{2016 July 21} \\
\hline Derek Breit & Morgan Hill, CA & 37.1133 & -121.7028 & Miss & Miss \\
\hline Bob Dunford & Naperville, IL & 41.759 & -88.1167 & Miss & Miss \\
\hline Brad Timerson & Newark, NY & 43.0066 & -77.1185 & Miss & Miss \\
\hline Kevin Green & Westport, CT & 41.1714 & -73.3278 & Miss & Miss \\
\hline Steve Conard & Gamber, MD & 39.4692 & -76.9516 & Miss & Miss \\
\hline Gary Frishkorn & Sykesville, MD & 39.2316 & -76.9929 & $7: 41: 59.05$ & $7: 42: 04.22$ \\
\hline Andy Scheck & Scaggsville, MD & 39.1497 & -76.8871 & $7: 41: 57.87$ & $7: 42: 06.68$ \\
\hline David Dunham/Joan Dunham & Greenbelt, MD & 38.9866 & -76.8694 & $7: 41: 56.97$ & no report \\
\hline Paul Maley & Clifton, TX & 31.6814 & -97.6744 & $7: 43: 26.99$ & $7: 43: 40.62$ \\
\hline Ned Smith & Trenton, GA & 34.893 & -85.4711 & $7: 42: 28.72$ & $7: 42: 42.92$ \\
\hline Ernie Iverson & Lufkin, TX & 31.3213 & -94.8444 & $7: 43: 15.48$ & $7: 43: 27.47$ \\
\hline
\end{tabular}

Table A.4

Date, number of positive and negative chords $\left(\#_{p}\right.$ and $\left.\#_{n}\right)$, average uncertainty in seconds $\left(\sigma_{s}\right)$ and kilometers $\left(\sigma_{k}\right)$, and RMS residuals with seconds, kilometers, and expressed in amount of standard deviation.

\begin{tabular}{llllllllll}
\hline & Date & $\begin{array}{l}\mathrm{UT} \\
(\mathrm{h})\end{array}$ & $\#_{p}$ & $\#_{n}$ & $\begin{array}{l}\sigma_{s} \\
(\mathrm{~s})\end{array}$ & $\begin{array}{l}\sigma_{k} \\
(\mathrm{~km})\end{array}$ & $\begin{array}{l}\mathrm{RMS}_{s} \\
(\mathrm{~s})\end{array}$ & $\begin{array}{l}\mathrm{RMS}_{k} \\
(\mathrm{~km})\end{array}$ & $\begin{array}{l}\mathrm{RMS}_{\sigma} \\
(\sigma)\end{array}$ \\
\hline 1 & $2004-09-05$ & $08: 54$ & 3 & 0 & 0.73 & 17.831 & 0.860 & 32.358 & 3.277 \\
2 & $2010-09-16$ & $04: 01$ & 1 & 1 & 0.05 & 0.267 & 0.066 & 0.995 & 1.318 \\
3 & $2015-01-01$ & $11: 26$ & 1 & 2 & 0.22 & 2.445 & 0.017 & 0.975 & 0.077 \\
4 & $2015-02-12$ & $11: 58$ & 2 & 0 & 0.20 & 6.728 & 1.230 & 22.304 & 8.171 \\
5 & $2015-05-06$ & $05: 23$ & 3 & 4 & 0.33 & 3.831 & 0.389 & 13.324 & 13.219 \\
6 & $2015-08-23$ & $04: 17$ & 6 & 5 & 0.15 & 4.994 & 0.072 & 8.039 & 2.362 \\
7 & 2016-07-21 & $07: 42$ & 5 & 5 & 0.56 & 5.060 & 0.579 & 18.920 & 3.067 \\
0 & Average & - & 3 & 2 & 0.32 & 5.880 & 0.459 & 13.845 & 4.499 \\
\hline
\end{tabular}


Table A.5

Astrometry of S1. Date, mid-observing time (UTC), telescope, camera, filter, astrometry ( $X$ is aligned with Right Ascension, and $Y$ with Declination, and $o$ and $c$ indices stand for observed and computed positions), and photometry (magnitude difference $\Delta M$ with uncertainty $\delta M$ ). PIs of these observations were: ${ }^{*}$ A. Storrs, ${ }^{a}$ J.-L. Margot, ${ }^{b}$ W. J. Merline, ${ }^{c}$ L. Sromovsky, ${ }^{d}$ F. Marchis, ${ }^{e}$ P. Rojo, and ${ }^{f}$ M. Marsset.

\begin{tabular}{|c|c|c|c|c|c|c|c|c|c|c|c|}
\hline Date & UTC & Tel. & Cam. & Filter & $\begin{array}{l}X_{o} \\
\text { (mas) }\end{array}$ & $\begin{array}{l}Y_{o} \\
\text { (mas) }\end{array}$ & $\begin{array}{l}X_{0-c} \\
\text { (mas) }\end{array}$ & $\begin{array}{l}Y_{O-c} \\
\text { (mas) }\end{array}$ & $\begin{array}{l}\sigma \\
\text { (mas) }\end{array}$ & $\begin{array}{l}\Delta M \\
(\mathrm{mag})\end{array}$ & $\begin{array}{l}\delta M \\
\text { (mag) }\end{array}$ \\
\hline 2001-03-01 & 05:48:13.0 & HST & ACS* & F439W & -573 & -84 & -22 & 1 & 10.00 & 0.00 & 0.00 \\
\hline 2001-03-01 & 06:00:12.9 & HST & ACS* & F791W & -565 & -70 & -20 & 13 & 10.00 & 0.00 & 0.00 \\
\hline 2002-05-08 & $10: 46: 01.0$ & Keck & $\mathrm{NIRC}^{a}$ & Kp & 472 & -189 & -4 & 15 & 9.94 & 6.34 & 1.50 \\
\hline 2003-06-06 & $14: 03: 06.0$ & Keck & $\mathrm{NIRC}^{b}$ & Ks & 402 & -214 & -5 & -8 & 9.94 & 6.53 & 1.18 \\
\hline 2003-06-06 & $14: 08: 23.2$ & Keck & $\mathrm{NIRC} 2^{b}$ & Ks & 406 & -213 & 1 & -8 & 9.94 & 7.18 & 0.45 \\
\hline 2003-06-06 & $14: 13: 30.2$ & Keck & $\mathrm{NIRC} 2^{b}$ & Ks & 402 & -218 & -1 & -12 & 9.94 & 6.31 & 0.23 \\
\hline 2003-07-15 & $07: 32: 50.4$ & VLT & $\mathrm{NACO}^{b}$ & $\mathrm{H}$ & -540 & 216 & 8 & 7 & 27.00 & 6.56 & 0.02 \\
\hline 2003-07-15 & $07: 37: 26.2$ & VLT & $\mathrm{NACO}^{b}$ & $\mathrm{H}$ & -536 & 222 & 10 & 12 & 27.00 & 6.34 & 0.14 \\
\hline 2003-08-14 & $10: 35: 08.0$ & Keck & $\mathrm{NIRC}^{a}$ & $\mathrm{H}$ & -183 & 227 & 5 & -4 & 9.94 & 5.04 & 3.68 \\
\hline 2003-08-15 & $08: 35: 22.2$ & Keck & $\mathrm{NIRC} 2^{c}$ & Kр & 554 & -62 & 5 & -1 & 9.94 & 6.67 & 0.18 \\
\hline 2003-08-15 & 08:39:27.2 & Keck & $\mathrm{NIRC}^{c}$ & Kp & 550 & -66 & 0 & -5 & 9.94 & 6.62 & 0.31 \\
\hline 2003-08-17 & $10: 50: 08.0$ & Keck & $\mathrm{NIRC}^{b}$ & $\mathrm{Kp}$ & -568 & 146 & 8 & 2 & 9.94 & 6.55 & 1.21 \\
\hline 2003-08-17 & $10: 53: 39.3$ & Keck & $\mathrm{NIRC} 2^{b}$ & Kp & -567 & 144 & 9 & 0 & 9.94 & 6.39 & 0.66 \\
\hline 2004-09-01 & 05:07:38.3 & VLT & $\mathrm{NACO}^{d}$ & Ks & 504 & -164 & 4 & 1 & 13.24 & 6.06 & 0.31 \\
\hline 2004-09-01 & $05: 17: 22.2$ & VLT & $\mathrm{NACO}^{d}$ & $\mathrm{H}$ & 510 & -165 & 5 & 0 & 13.24 & 6.34 & 0.24 \\
\hline 2004-09-01 & 08:06:43.4 & VLT & $\mathrm{NACO}^{d}$ & Ks & 576 & -169 & 8 & -1 & 13.24 & 6.98 & 0.43 \\
\hline 2004-09-03 & $06: 51: 57.5$ & VLT & $\mathrm{NACO}^{d}$ & Ks & -623 & 166 & -21 & 2 & 13.24 & 6.76 & 0.70 \\
\hline 2004-09-05 & $04: 28: 20.2$ & VLT & $\mathrm{NACO}^{d}$ & Ks & 624 & -163 & 6 & 0 & 13.24 & 6.73 & 0.09 \\
\hline 2004-09-08 & $06: 41: 20.1$ & VLT & $\mathrm{NACO}^{d}$ & Ks & 211 & -120 & 4 & -8 & 13.24 & 6.95 & 0.59 \\
\hline 2004-09-11 & $04: 34: 26.2$ & VLT & $\mathrm{NACO}^{d}$ & Ks & -539 & 87 & -5 & -9 & 13.24 & 7.09 & 1.15 \\
\hline 2004-09-13 & $03: 42: 52.5$ & VLT & $\mathrm{NACO}^{d}$ & Ks & 470 & -75 & 1 & 0 & 13.24 & 7.23 & 1.22 \\
\hline $2004-09-13$ & $05: 47: 28.2$ & VLT & $\mathrm{NACO}^{d}$ & Ks & 386 & -46 & -15 & 6 & 13.24 & 6.08 & 1.51 \\
\hline 2004-09-14 & 04:09:30.3 & VLT & $\mathrm{NACO}^{d}$ & Ks & -500 & 153 & -9 & -1 & 13.24 & 6.59 & 0.14 \\
\hline 2004-09-15 & $04: 18: 34.3$ & VLT & $\mathrm{NACO}^{d}$ & Ks & -321 & 35 & 0 & 4 & 13.24 & 6.28 & 1.20 \\
\hline 2004-09-15 & $04: 26: 56.5$ & VLT & $\mathrm{NACO}^{d}$ & $\mathrm{H}$ & -315 & 36 & 0 & 6 & 13.24 & 7.30 & 0.69 \\
\hline $2004-10-07$ & $02: 02: 03.0$ & VLT & $\mathrm{NACO}^{d}$ & Ks & -540 & 123 & 4 & -4 & 13.24 & 8.49 & 1.55 \\
\hline 2004-10-08 & $02: 22: 38.3$ & VLT & $\mathrm{NACO}^{d}$ & Ks & 356 & -106 & 3 & 0 & 13.24 & 8.22 & 2.62 \\
\hline $2004-10-08$ & $04: 47: 21.2$ & VLT & $\mathrm{NACO}^{d}$ & Ks & 435 & -125 & 3 & -1 & 13.24 & 7.07 & 1.05 \\
\hline $2004-10-20$ & $00: 39: 22.2$ & VLT & $\mathrm{NACO}^{d}$ & Ks & 553 & -136 & 8 & 0 & 13.24 & 6.55 & 0.19 \\
\hline 2004-11-02 & 07:36:13.0 & Gemini & NIRI $^{b}$ & Kр & -344 & 88 & -2 & 0 & 21.90 & 6.49 & 0.62 \\
\hline 2004-11-02 & $07: 38: 36.9$ & Gemini & $\mathrm{NIRI}^{b}$ & Kp & -340 & 90 & 0 & 2 & 21.90 & 6.40 & 0.29 \\
\hline 2004-11-05 & 08:09:18.1 & Gemini & $\mathrm{NIRI}^{b}$ & Kp & -538 & 138 & -6 & 1 & 21.90 & 5.95 & 0.16 \\
\hline $2005-12-21$ & 09:05:51.5 & Gemini & $\mathrm{NIRI}^{d}$ & Ks & 684 & 0 & 8 & -6 & 21.90 & 6.53 & 0.02 \\
\hline 2006-01-01 & $10: 17: 12.1$ & Gemini & $\mathrm{NIRI}^{d}$ & Ks & 651 & -35 & 6 & -7 & 21.90 & 6.71 & 0.17 \\
\hline 2006-01-09 & 05:20:11.1 & Gemini & $\mathrm{NIRI}^{d}$ & Ks & 557 & 116 & 17 & 1 & 21.90 & 5.86 & 0.34 \\
\hline 2006-01-16 & 05:16:51.5 & Gemini & $\mathrm{NIRI}^{d}$ & Ks & 619 & -17 & 22 & 31 & 21.90 & 5.85 & 0.28 \\
\hline 2009-06-07 & $10: 29: 14.1$ & Keck & $\mathrm{NIRC} 2^{b}$ & $\mathrm{H}$ & 510 & 54 & 0 & -1 & 9.94 & 6.56 & 0.44 \\
\hline 2009-06-07 & $10: 32: 18.1$ & Keck & $\mathrm{NIRC} 2^{b}$ & $\mathrm{H}$ & 511 & 55 & 0 & 0 & 9.94 & 6.49 & 0.50 \\
\hline 2009-06-07 & $10: 54: 08.0$ & Keck & $\mathrm{NIRC} 2^{b}$ & Кр & 516 & 55 & -4 & 7 & 9.94 & 6.23 & 0.44 \\
\hline 2009-06-07 & $11: 23: 04.0$ & Keck & $\mathrm{NIRC}^{b}$ & $\mathrm{Kp}$ & 530 & 44 & -2 & 5 & 9.94 & 6.56 & 1.07 \\
\hline 2009-06-07 & $11: 25: 56.5$ & Keck & $\mathrm{NIRC} 2^{b}$ & Kp & 530 & 39 & -3 & 0 & 9.94 & 6.66 & 0.17 \\
\hline 2009-08-16 & $06: 47: 02.0$ & Keck & $\mathrm{NIRC}^{d}$ & FeII & -36 & 239 & 6 & 0 & 9.94 & 6.99 & 1.19 \\
\hline 2010-08-15 & 08:07:02.0 & Gemini & $\mathrm{NIRI}^{d}$ & Кр & -421 & 182 & 0 & 14 & 21.90 & 5.84 & 0.08 \\
\hline $2010-08-15$ & $08: 16: 53.5$ & Gemini & $\mathrm{NIRI}^{d}$ & $\mathrm{Kp}$ & -412 & 181 & 4 & 13 & 21.90 & 6.40 & 0.05 \\
\hline $2010-08-28$ & 08:49:11.1 & Gemini & $\mathrm{NIRI}^{d}$ & Kp & 379 & -189 & 0 & -14 & 21.90 & 6.05 & 0.28 \\
\hline $2010-08-28$ & 08:54:01.0 & Gemini & $\mathrm{NIRI}^{d}$ & Kp & 378 & -186 & 0 & -12 & 21.90 & 6.52 & 0.47 \\
\hline 2010-09-02 & $06: 45: 32.3$ & Gemini & $\mathrm{NIRI}^{d}$ & Kp & -588 & 157 & -3 & 9 & 21.90 & 6.02 & 0.10 \\
\hline
\end{tabular}


Table A.5 (continued)

\begin{tabular}{|c|c|c|c|c|c|c|c|c|c|c|c|}
\hline Date & UTC & Tel. & Cam. & Filter & $\begin{array}{l}X_{o} \\
\text { (mas) }\end{array}$ & $\begin{array}{l}Y_{o} \\
\text { (mas) }\end{array}$ & $\begin{array}{l}X_{o-c} \\
\text { (mas) }\end{array}$ & $\begin{array}{l}Y_{o-c} \\
\text { (mas) }\end{array}$ & $\begin{array}{l}\sigma \\
\text { (mas) }\end{array}$ & $\begin{array}{l}\Delta M \\
\text { (mag) }\end{array}$ & $\begin{array}{l}\delta M \\
\text { (mag) }\end{array}$ \\
\hline $2010-10-31$ & 05:58:48.4 & Gemini & $\mathrm{NIRI}^{b}$ & Kp & -271 & -8 & 27 & -8 & 21.90 & 6.67 & 0.05 \\
\hline $2010-10-31$ & $06: 03: 23.2$ & Gemini & NIRI $^{b}$ & Kp & -290 & -2 & 10 & -3 & 21.90 & 6.87 & 0.06 \\
\hline 2011-09-27 & 05:04:41.0 & VLT & $\mathrm{NACO}^{d}$ & $\mathrm{H}$ & -287 & 236 & 5 & 12 & 13.24 & 6.39 & 1.09 \\
\hline 2011-09-29 & $05: 21: 18.0$ & VLT & $\mathrm{NACO}^{d}$ & $\mathrm{H}$ & 440 & -217 & -3 & -8 & 13.24 & 7.04 & 1.18 \\
\hline $2011-11-08$ & $03: 21: 35.3$ & VLT & $\mathrm{NACO}^{e}$ & $\mathrm{H}$ & -438 & -61 & -6 & 0 & 13.24 & 6.66 & 0.06 \\
\hline $2011-11-10$ & $01: 22: 04.0$ & VLT & $\mathrm{NACO}^{e}$ & $\mathrm{H}$ & 386 & 93 & 5 & 17 & 13.24 & 7.35 & 0.17 \\
\hline $2015-05-29$ & $04: 38: 46.4$ & VLT & SPHERE $^{f}$ & Ks & -188 & 240 & 3 & 0 & 12.26 & 6.29 & 0.06 \\
\hline $2015-05-29$ & $04: 38: 46.4$ & VLT & SPHERE $^{f}$ & $\mathrm{YJH}$ & -184 & 237 & 6 & -3 & 7.40 & 6.28 & 0.07 \\
\hline $2015-05-29$ & $04: 51: 27.2$ & VLT & SPHERE $f$ & $\mathrm{YJH}$ & -176 & 239 & 4 & -1 & 7.40 & 6.30 & 0.13 \\
\hline $2015-05-29$ & $04: 51: 27.2$ & VLT & SPHERE $f$ & Ks & -180 & 241 & 1 & 0 & 12.26 & 6.26 & 0.09 \\
\hline $2015-05-29$ & $05: 07: 36.3$ & VLT & SPHERE $^{f}$ & Ks & -166 & 245 & 3 & 2 & 12.26 & 6.26 & 0.06 \\
\hline $2015-05-29$ & 05:07:36.3 & VLT & SPHERE $^{f}$ & $\mathrm{YJH}$ & -164 & 241 & 5 & -1 & 7.40 & 6.35 & 0.09 \\
\hline $2015-05-29$ & $05: 15: 13.1$ & VLT & SPHERE $^{f}$ & $\mathrm{YJH}$ & -157 & 242 & 6 & 0 & 7.40 & 6.43 & 0.30 \\
\hline $2015-05-29$ & $05: 15: 13.1$ & VLT & SPHERE $^{f}$ & Ks & -158 & 245 & 5 & 2 & 12.26 & 6.35 & 0.17 \\
\hline $2015-05-29$ & $05: 25: 55.5$ & VLT & SPHERE $^{f}$ & Ks & -152 & 245 & 3 & 1 & 12.26 & 6.36 & 0.16 \\
\hline $2015-05-29$ & $05: 28: 59.5$ & VLT & SPHERE $^{f}$ & $\mathrm{YJH}$ & -148 & 243 & 4 & 0 & 7.40 & 6.49 & 0.16 \\
\hline $2015-05-29$ & 05:28:59.5 & VLT & SPHERE $^{f}$ & Ks & -150 & 247 & 3 & 2 & 12.26 & 6.34 & 0.08 \\
\hline $2015-05-29$ & 05:32:04.0 & VLT & SPHERE $^{f}$ & Ks & -148 & 245 & 2 & 0 & 12.26 & 6.42 & 0.09 \\
\hline $2015-05-29$ & $05: 32: 04.0$ & VLT & SPHERE $^{f}$ & $\mathrm{YJH}$ & -146 & 243 & 4 & -1 & 7.40 & 6.51 & 0.17 \\
\hline 2016-07-02 & $08: 47: 22.2$ & VLT & SPHERE $^{f}$ & YJH & -279 & -90 & -5 & -4 & 7.40 & 6.68 & 0.28 \\
\hline 2016-07-12 & 05:04:19.4 & VLT & SPHERE $^{f}$ & $\mathrm{YJH}$ & 601 & -129 & -9 & 1 & 7.40 & 6.55 & 0.19 \\
\hline 2016-07-12 & $05: 11: 41.7$ & VLT & SPHERE $f$ & YJH & 601 & -130 & -10 & 1 & 7.40 & 6.51 & 0.06 \\
\hline $2016-07-12$ & $05: 19: 03.9$ & VLT & SPHERE $^{f}$ & $\mathrm{YJH}$ & 601 & -129 & -10 & 4 & 7.40 & 6.49 & 0.03 \\
\hline $2016-07-28$ & 05:50:56.0 & VLT & SPHERE $^{f}$ & $\mathrm{YJH}$ & -208 & -138 & 7 & -5 & 7.40 & 6.96 & 0.24 \\
\hline $2016-07-28$ & $05: 57: 12.3$ & VLT & SPHERE $f$ & YJH & -212 & -137 & 8 & -6 & 7.40 & 7.10 & 0.06 \\
\hline $2016-07-28$ & $06: 03: 30.1$ & VLT & SPHERE $f$ & $\mathrm{YJH}$ & -216 & -135 & 7 & -5 & 7.40 & 7.07 & 0.19 \\
\hline $2016-07-30$ & $01: 37: 12.1$ & VLT & SPHERE $^{f}$ & $\mathrm{YJH}$ & 192 & 141 & -1 & 0 & 7.40 & 6.90 & 0.20 \\
\hline 2016-07-30 & $01: 44: 17.2$ & VLT & SPHERE $^{f}$ & YJH & 194 & 135 & -4 & -4 & 7.40 & 6.78 & 0.23 \\
\hline 2016-07-30 & $01: 51: 22.2$ & VLT & SPHERE $^{f}$ & $\mathrm{YJH}$ & 199 & 135 & -4 & -2 & 7.40 & 6.65 & 0.44 \\
\hline 2016-08-11 & $00: 18: 43.4$ & VLT & SPHERE $f$ & $\mathrm{YJH}$ & 579 & -159 & -9 & 6 & 7.40 & 6.18 & 0.07 \\
\hline 2016-08-11 & $02: 41: 34.1$ & VLT & SPHERE $^{f}$ & YJH & 559 & -189 & -6 & 5 & 7.40 & 6.54 & 0.23 \\
\hline 2016-08-11 & $02: 48: 41.5$ & VLT & SPHERE $f$ & $\mathrm{YJH}$ & 560 & -189 & -3 & 6 & 7.40 & 6.43 & 0.08 \\
\hline 2016-08-11 & $02: 55: 50.8$ & VLT & SPHERE $^{f}$ & YJH & 556 & -194 & -5 & 2 & 7.40 & 6.43 & 0.07 \\
\hline & & & & & \multicolumn{2}{|c|}{ Average } & 1 & 0 & 18 & 6.50 & 0.46 \\
\hline & & & & & \multicolumn{3}{|c|}{ Standard deviation 8} & 7 & 7 & 0.28 & 0.61 \\
\hline
\end{tabular}

Table A.6

Astrometry of S2. Date, mid-observing time (UTC), telescope, camera, filter, astrometry ( $X$ is aligned with Right Ascension, and $Y$ with Declination, and $o$ and $c$ indices stand for observed and computed positions), and photometry (magnitude difference $\Delta M$ with uncertainty $\delta M$ ). The PI of these observations was M. Marsset.

\begin{tabular}{|c|c|c|c|c|c|c|c|c|c|c|c|}
\hline Date & UTC & Tel. & Cam. & Filter & $\begin{array}{l}X_{o} \\
\text { (mas) }\end{array}$ & $\begin{array}{l}Y_{o} \\
\text { (mas) }\end{array}$ & $\begin{array}{l}X_{o-c} \\
\text { (mas) }\end{array}$ & $\begin{array}{l}Y_{o-c} \\
\text { (mas) }\end{array}$ & $\begin{array}{l}\sigma \\
\text { (mas) }\end{array}$ & $\begin{array}{l}\Delta M \\
(\mathrm{mag})\end{array}$ & $\begin{array}{l}\delta M \\
\text { (mag) }\end{array}$ \\
\hline $2015-05-29$ & 04:38:46.4 & VLT & SPHERE & YJH & 87 & 140 & -3 & 10 & 7.40 & 8.95 & 1.40 \\
\hline $2015-05-29$ & $04: 51: 27.2$ & VLT & SPHERE & YJH & 102 & 141 & 1 & 7 & 7.40 & 8.65 & 0.25 \\
\hline $2015-05-29$ & $05: 07: 36.3$ & VLT & SPHERE & $\mathrm{YJH}$ & 111 & 137 & -2 & -1 & 7.40 & 8.43 & 1.53 \\
\hline $2015-05-29$ & $05: 15: 13.1$ & VLT & SPHERE & YJH & 121 & 142 & 1 & 1 & 7.40 & 8.66 & 0.60 \\
\hline $2015-05-29$ & 05:32:04.0 & VLT & SPHERE & YJH & 135 & 136 & 2 & -9 & 7.40 & 8.83 & 1.59 \\
\hline $2016-07-12$ & 05:04:19.4 & VLT & SPHERE & $\mathrm{YJH}$ & -271 & 115 & 10 & 0 & 7.40 & 9.16 & 0.82 \\
\hline 2016-07-12 & $05: 11: 41.7$ & VLT & SPHERE & $\mathrm{YJH}$ & -275 & 113 & 2 & -5 & 7.40 & 9.53 & 1.23 \\
\hline 2016-07-12 & 05:19:03.9 & VLT & SPHERE & YJH & -272 & 119 & 0 & -2 & 7.40 & 9.34 & 0.95 \\
\hline $2016-07-30$ & $01: 37: 12.1$ & VLT & SPHERE & $\mathrm{YJH}$ & -295 & 104 & -2 & 5 & 7.40 & 9.32 & 0.33 \\
\hline $2016-07-30$ & 01:44:17.2 & VLT & SPHERE & YJH & -295 & 103 & -6 & 0 & 7.40 & 9.23 & 0.20 \\
\hline \multirow[t]{3}{*}{$2016-07-30$} & $01: 51: 22.2$ & VLT & SPHERE & YJH & -288 & 102 & -3 & -3 & 7.40 & 9.53 & 1.69 \\
\hline & & & & & \multicolumn{2}{|c|}{ Average } & 0 & 0 & 10 & 9.05 & 0.96 \\
\hline & & & & & \multicolumn{3}{|c|}{ Standard deviation 4} & 5 & 0 & 0.32 & 0.56 \\
\hline
\end{tabular}




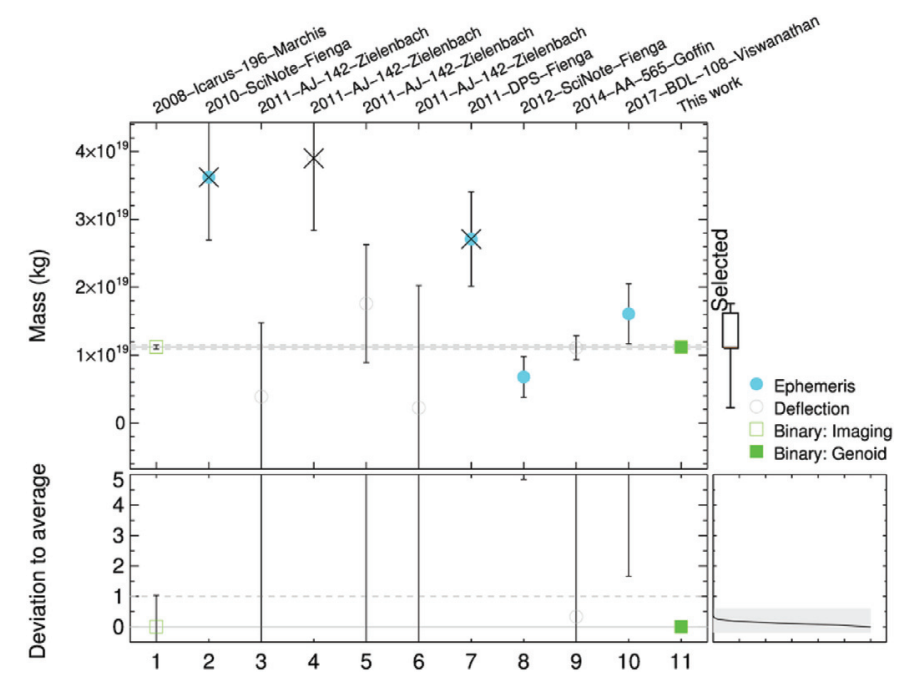

Fig. B.1. Mass estimates of (107) Camilla gathered from the literature, see Table B.1 for details.

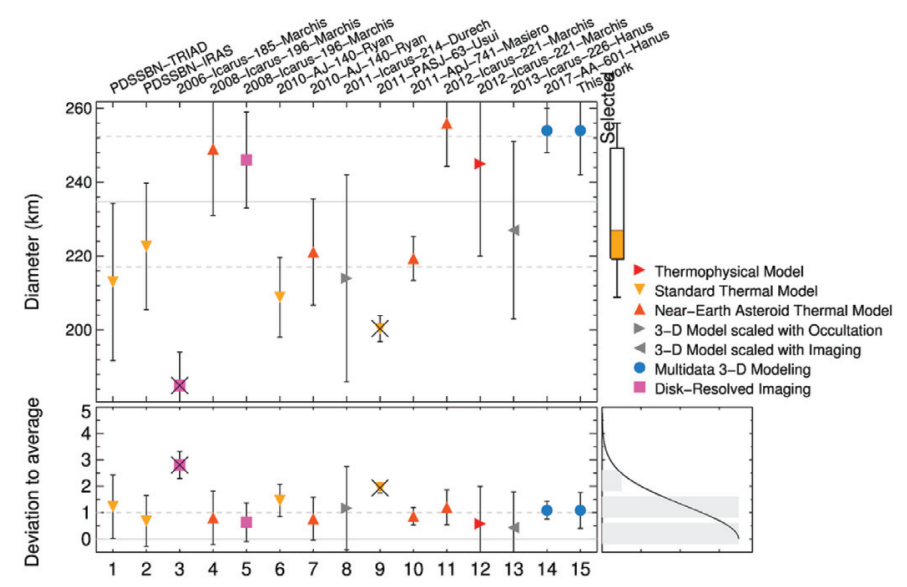

Fig. B.2. Diameter estimates of (107) Camilla gathered from the literature, see Table B.2 for details.

Table B.1

The mass estimates $(\mathcal{M})$ of (107) Camilla collected from the literature. For each, the $3 \sigma$ uncertainty, method, selection flag, and bibliographic reference are reported. The methods are BIN: Binary, DEFL: Deflection, ернем: Ephemeris. ....".

\begin{tabular}{|c|c|c|c|c|}
\hline \# & $\begin{array}{l}\text { Mass }(\mathcal{M}) \\
(\mathrm{kg})\end{array}$ & Method & Sel. & Reference \\
\hline 1 & $(1.12 \pm 0.09) \times 10^{19}$ & BIM & $\sqrt{ }$ & Marchis et al. (2008a) \\
\hline 2 & $(36.20 \pm 27.72) \times 10^{18}$ & EPHEM & $x$ & Fienga et al. (2010) \\
\hline 3 & $3.88_{-3.88}^{+32.70} \times 10^{18}$ & DEFL & $\sqrt{ }$ & Zielenbach (2011) \\
\hline 4 & $(39.00 \pm 31.80) \times 10^{18}$ & DEFL & $x$ & Zielenbach (2011) \\
\hline 5 & $(17.60 \pm 26.07) \times 10^{18}$ & DEFL & $\sqrt{ }$ & Zielenbach (2011) \\
\hline 6 & $2.25_{-2.25}^{+54.00} \times 10^{18}$ & DEFL & $\sqrt{ }$ & Zielenbach (2011) \\
\hline 7 & $(27.10 \pm 20.88) \times 10^{18}$ & EPHEM & $x$ & Fienga et al. (2011) \\
\hline 8 & $(6.79 \pm 9.00) \times 10^{18}$ & EPHEM & $\sqrt{ }$ & Fienga et al. (2013) \\
\hline 9 & $(11.10 \pm 5.37) \times 10^{18}$ & DEFL & $\sqrt{ }$ & Goffin (2014) \\
\hline 10 & $(16.10 \pm 13.26) \times 10^{18}$ & EPHEM & $\sqrt{ }$ & Viswanathan et al. (2017) \\
\hline \multirow[t]{2}{*}{11} & $(1.12 \pm 0.01) \times 10^{19}$ & BIN & $\sqrt{ }$ & This work \\
\hline & $(1.12 \pm 0.09) \times 10^{19}$ & \multicolumn{2}{|c|}{ Average } & \\
\hline
\end{tabular}


Table B.2

The diameter estimates $(\mathcal{D})$ of (107) Camilla collected from the literature. For each, the $3 \sigma$ uncertainty, method, selection flag, and bibliographic reference are reported. The methods are IM: Disk-Resolved Imaging, ADAM/KOALA: Multidata 3-D Modeling, LCIMG: 3-D Model scaled with Imaging, Lcocc: 3-D Model scaled with Occultation, NEATM: Near-Earth Asteroid Thermal Model, sTM: Standard Thermal Model, TPM: Thermophysical Model.

\begin{tabular}{|c|c|c|c|c|c|}
\hline \# & $\begin{array}{l}\mathcal{D} \\
(\mathrm{km})\end{array}$ & $\begin{array}{l}\delta \mathcal{D} \\
(\mathrm{km})\end{array}$ & Method & Sel. & Reference \\
\hline 1 & 213.00 & 63.90 & STM & $\sqrt{ }$ & Morrison and Zellner (2007) \\
\hline 2 & 222.62 & 51.30 & STM & $\sqrt{ }$ & Tedesco et al. (2004) \\
\hline 3 & 185.00 & 27.00 & IM & $x$ & Marchis et al. (2006) \\
\hline 4 & 249.00 & 54.00 & NEATM & $\sqrt{ }$ & Marchis et al. (2008a) \\
\hline 5 & 246.00 & 39.00 & IM & $\sqrt{ }$ & Marchis et al. (2008a) \\
\hline 6 & 208.85 & 32.37 & STM & $\sqrt{ }$ & Ryan and Woodward (2010) \\
\hline 7 & 221.10 & 43.11 & NEATM & $\sqrt{ }$ & Ryan and Woodward (2010) \\
\hline 8 & 214.00 & 84.00 & LCOCC & $\sqrt{ }$ & Durech et al. (2011) \\
\hline 9 & 200.37 & 10.53 & STM & $x$ & Usui et al. (2011) \\
\hline 10 & 219.37 & 17.82 & NEATM & $\sqrt{ }$ & Masiero et al. (2011) \\
\hline 11 & 256.00 & 35.00 & NEATM & $\sqrt{ }$ & Marchis et al. (2012) \\
\hline 12 & 245.00 & 75.00 & TPM & $\sqrt{ }$ & Marchis et al. (2012) \\
\hline 13 & 227.00 & 72.00 & LCIMG & $\sqrt{ }$ & Hanuš et al. (2013) \\
\hline 14 & 254.00 & 18.00 & ADAM & $\sqrt{ }$ & Hanuš et al. (2017) \\
\hline \multirow[t]{2}{*}{15} & 254.00 & 36.00 & KOALA & $\sqrt{ }$ & This work \\
\hline & 234.72 & 53.11 & \multicolumn{2}{|c|}{ Average } & \\
\hline
\end{tabular}

Table B.3

Spherical-equivalent diameter $\left(\mathcal{D}_{e}\right)$ of the shape model of Camilla projected on the plane of the sky as seen from IRAS, AKARI, and WISE Tedesco et al. (2004); Usui et al. (2011); Masiero et al. (2011). Owing to the elongated shape of Camilla, the 2-D diameter often underestimates the spherical-volume equivalent diameter.

\begin{tabular}{|c|c|c|}
\hline & Epoch (UTC) & $\mathcal{D}_{e}$ \\
\hline IRAS & $\begin{array}{l}\text { 1983-03-14T12:55 } \\
\text { 1983-03-14T14:26 } \\
\text { 1983-03-22T01:11 } \\
\text { 1983-03-21T23:28 } \\
\text { 1983-03-29T20:25 } \\
\text { 1983-03-30T12:04 } \\
\text { 1983-09-30T08:21 } \\
\text { 1983-09-30T10:04 } \\
\text { 1983-09-30T06:38 } \\
\text { Average } \\
\text { Standard deviation }\end{array}$ & $\begin{array}{l}242.1 \\
265.3 \\
257.8 \\
266.5 \\
266.0 \\
236.6 \\
244.5 \\
263.5 \\
243.2 \\
254.0 \\
12.2\end{array}$ \\
\hline WISE & $\begin{array}{l}\text { 2010-05-18T06:50 } \\
\text { 2010-05-18T10:01 } \\
\text { 2010-05-18T13:11 } \\
\text { 2010-05-18T19:32 } \\
\text { 2010-05-18T21:08 } \\
\text { 2010-05-18T22:43 } \\
\text { 2010-05-19T00:18 } \\
\text { 2010-05-19T01:53 } \\
\text { 2010-05-19T03:29 } \\
\text { 2010-05-19T06:39 } \\
\text { 2010-05-19T09:50 } \\
\text { 2010-05-19T13:00 } \\
\text { 2010-11-05T05:36 } \\
2010-11-05 T 15: 08 \\
\text { 2010-11-05T16:43 } \\
\text { 2010-11-05T18:18 } \\
\text { 2010-11-05T18:18 } \\
\text { 2010-11-05T23:04 } \\
\text { 2010-11-05T23:04 } \\
\text { Average } \\
\text { Standard deviation }\end{array}$ & $\begin{array}{l}241.8 \\
228.0 \\
261.5 \\
222.3 \\
255.9 \\
256.0 \\
220.7 \\
259.9 \\
252.9 \\
262.7 \\
220.5 \\
244.1 \\
238.3 \\
248.2 \\
259.7 \\
225.1 \\
225.1 \\
222.8 \\
222.8 \\
240.4 \\
16.3\end{array}$ \\
\hline AKARI & $\begin{array}{l}2006-11-05 T 21: 59 \\
2006-11-05 T 23: 38 \\
\text { 2007-04-29T08:45 } \\
\text { 2007-04-29T10:24 } \\
\text { 2007-04-29T20:21 } \\
\text { Average } \\
\text { Standard deviation }\end{array}$ & $\begin{array}{l}232.9 \\
236.0 \\
240.4 \\
265.1 \\
264.7 \\
247.8 \\
15.8\end{array}$ \\
\hline
\end{tabular}




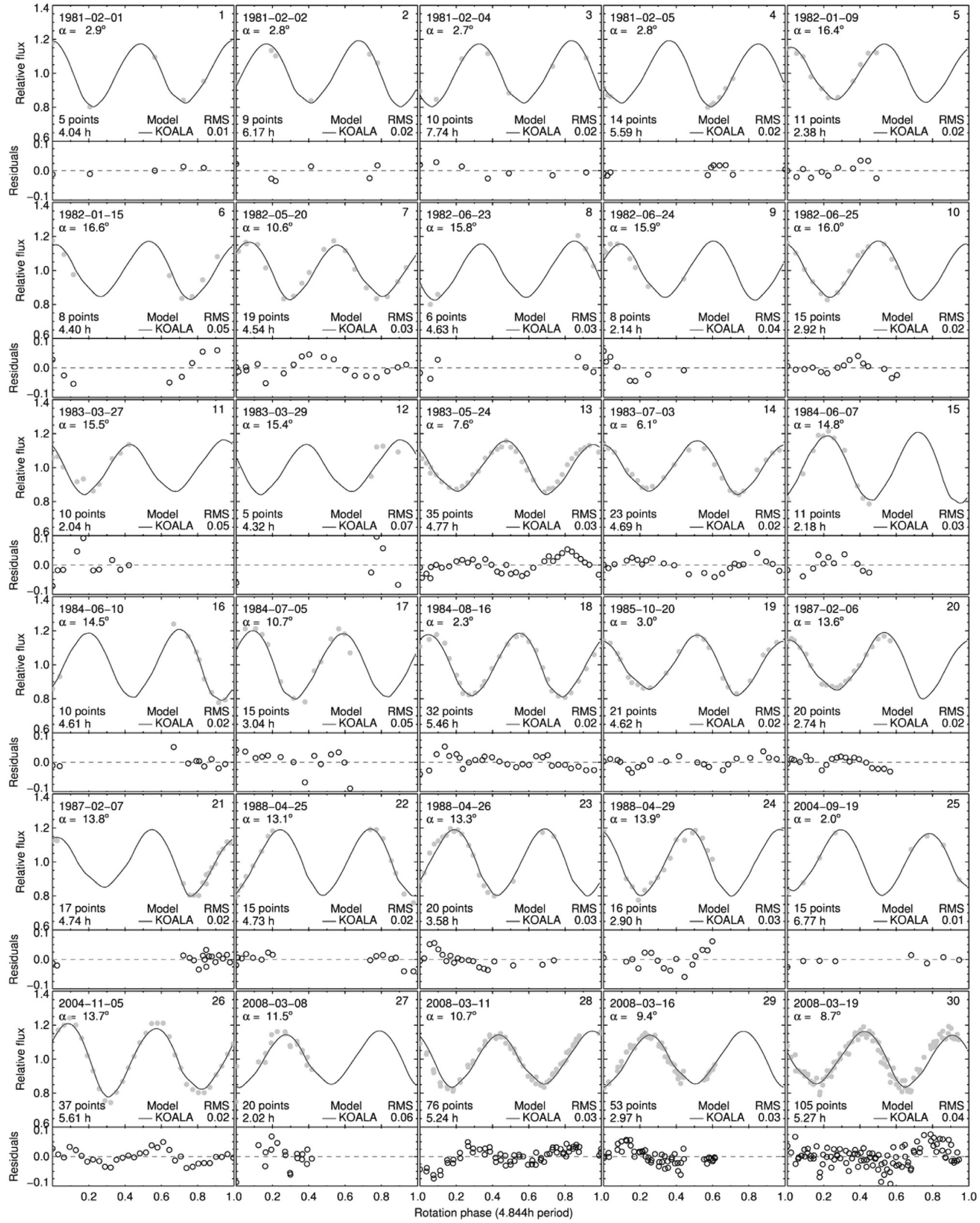

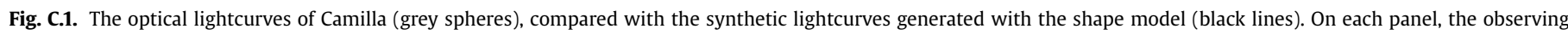

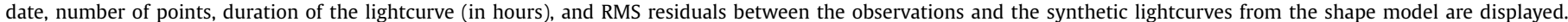
In many cases, measurement uncertainties are not provided by the observers but can be estimated from the spread of measurements. 


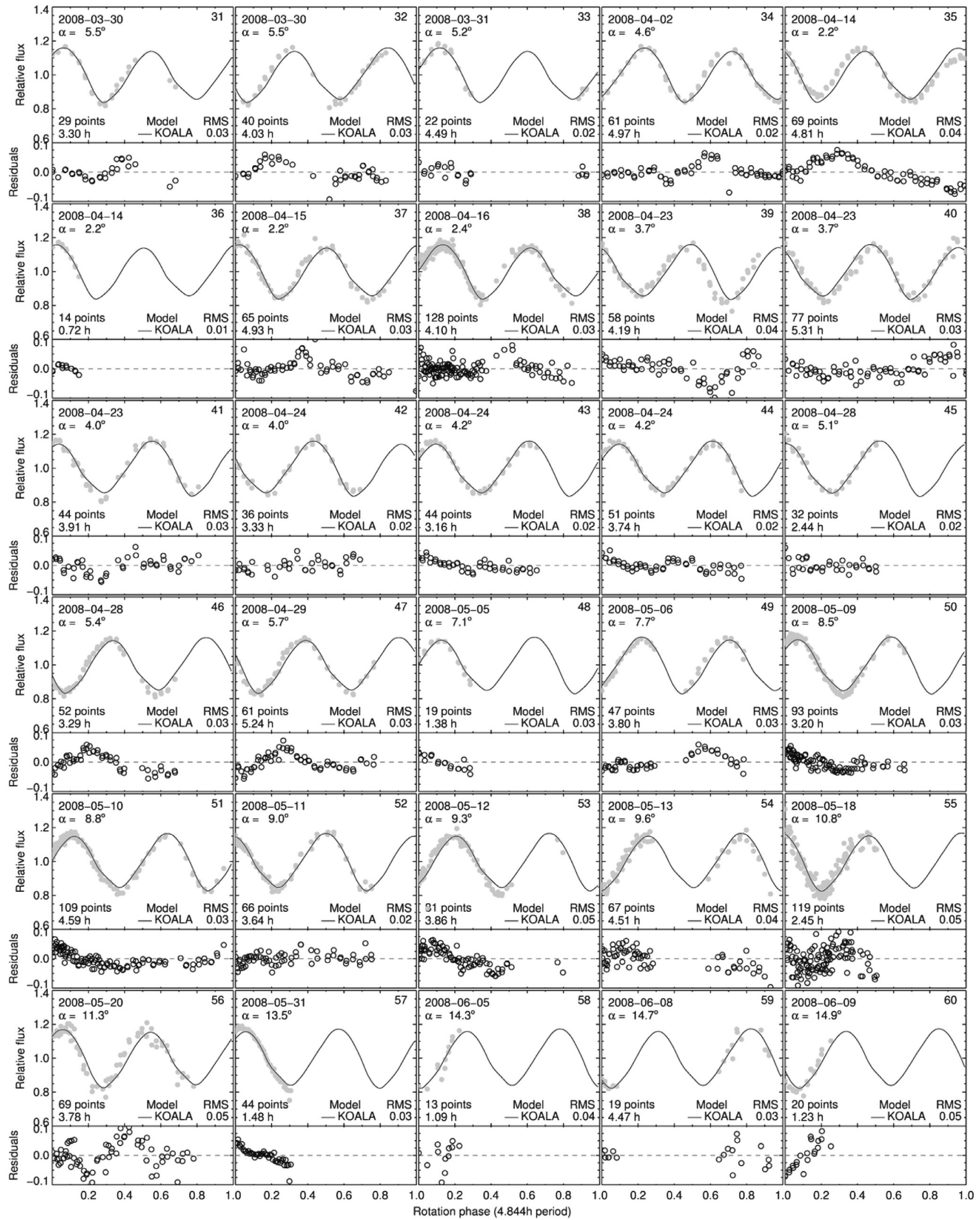

Fig. C.1. Suite of all lightcurve plots, as described in Fig. 6. (Continued). 


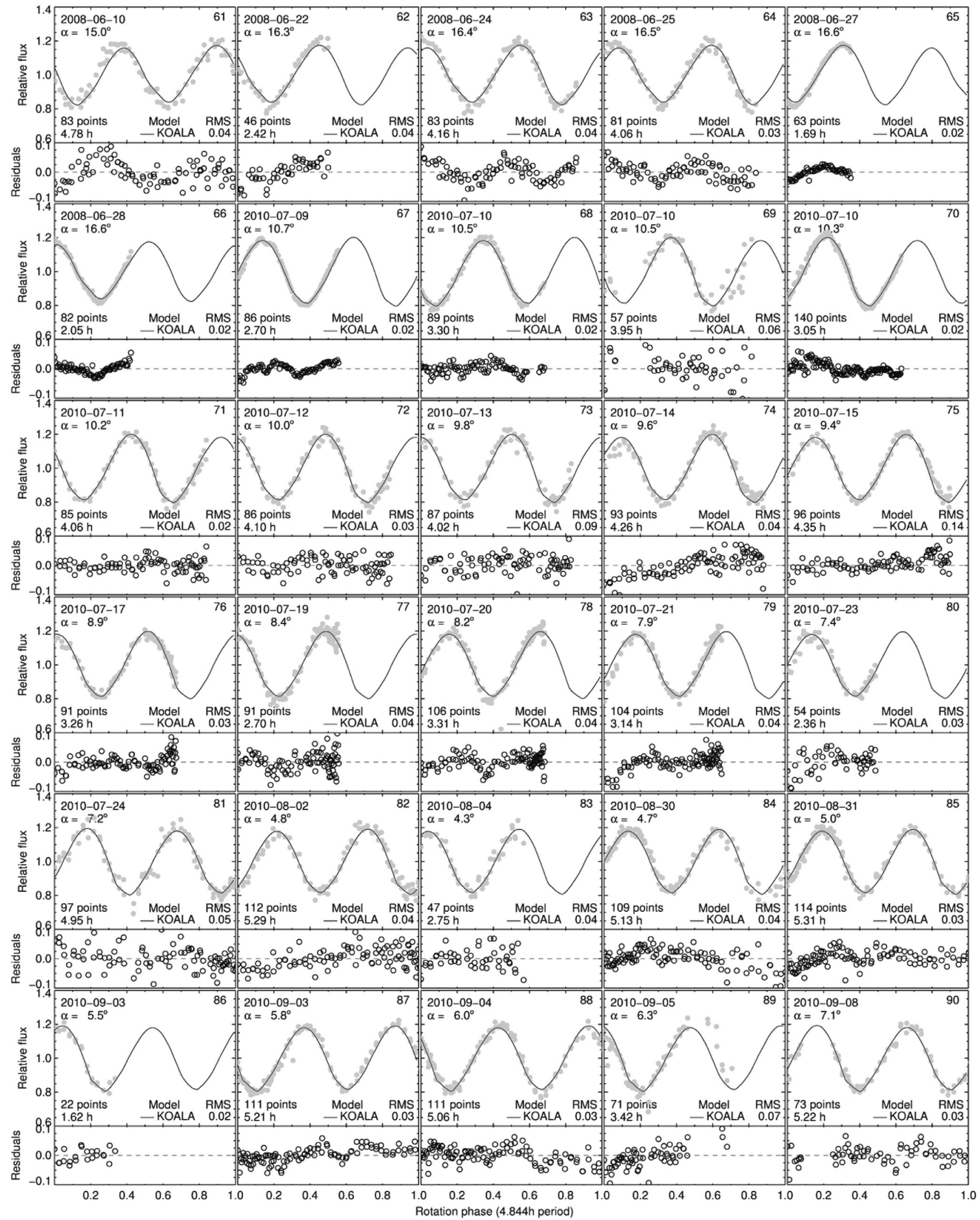

Fig. C.1. Continued 


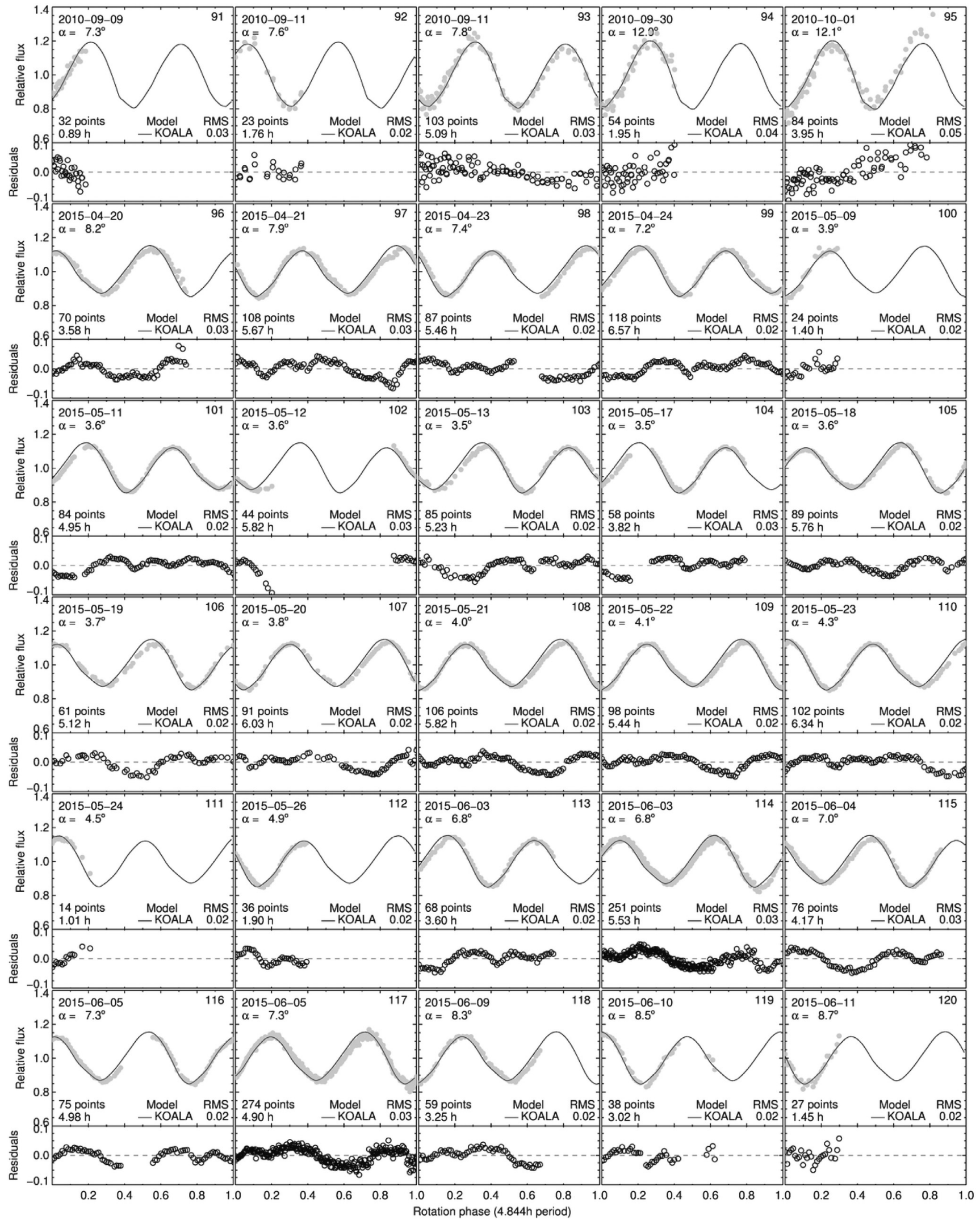

Fig. C.1. Continued 


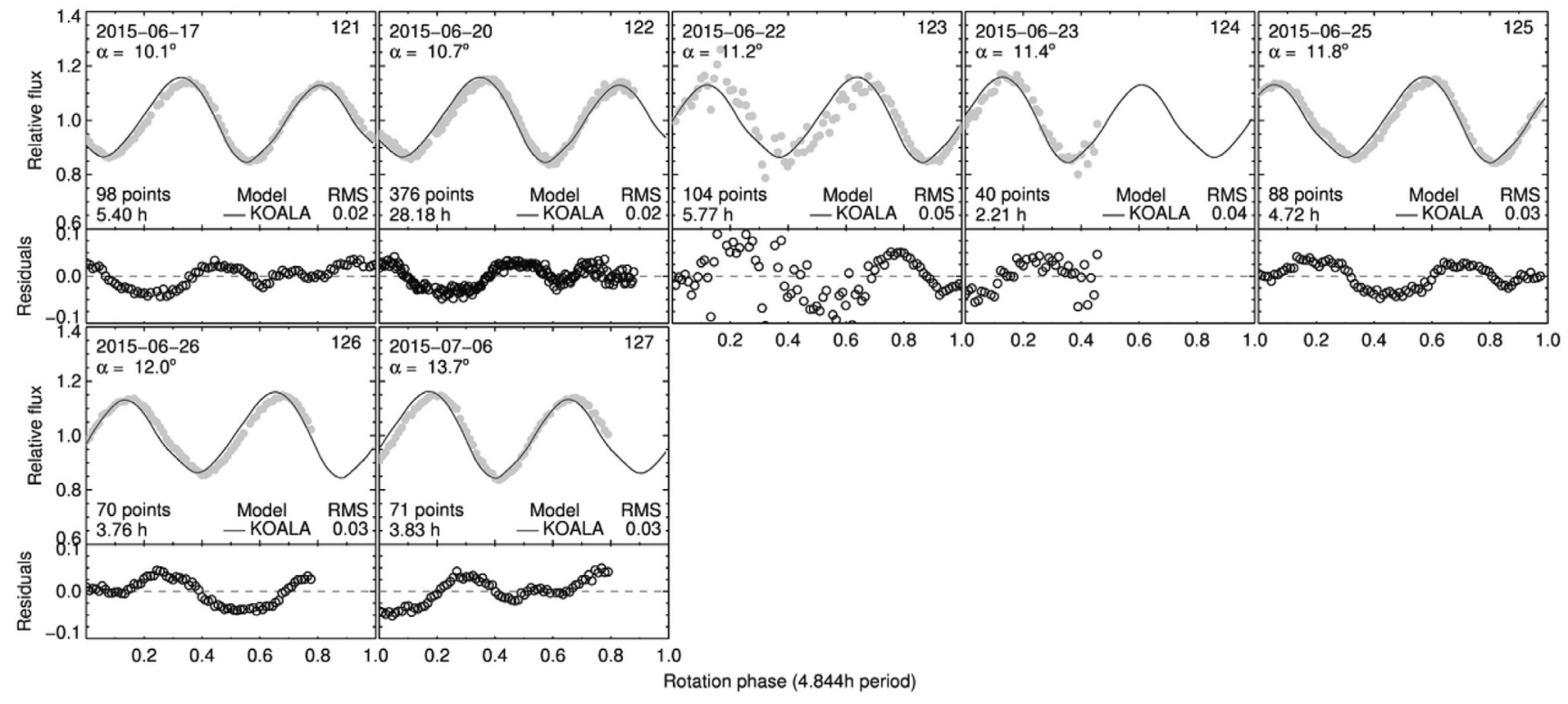

Fig. C.1. Continued

\section{Appendix D. Future occultations}

Table D.1

Selection of stellar occultations by (107) Camilla scheduled for the next 3 years. For each, we report the mean epoch of the event, the identifier of the UCAC-2 star and its magnitude $\left(m_{\star}\right)$, the expected drop in magnitude $(\Delta m)$, the expected maximum duration of the event $(\Delta t)$, the uncertainty $(3 \sigma)$ on the position of both satellites S1 and S2 at the date, projected on Earth, and the main area of visibility (location).

\begin{tabular}{llllllll}
\hline $\begin{array}{l}\text { Mean epoch } \\
\text { (UTC) }\end{array}$ & $\begin{array}{l}\text { Star } \\
(\mathrm{UCAC2})\end{array}$ & $\begin{array}{l}\mathrm{m} * \\
(\mathrm{mag})\end{array}$ & $\begin{array}{l}\Delta m \\
(\mathrm{mag})\end{array}$ & $\begin{array}{l}\Delta t \\
(\mathrm{~s})\end{array}$ & $\begin{array}{l}\mathrm{S} 1 \\
(\mathrm{~km})\end{array}$ & $\begin{array}{l}\mathrm{S} 2 \\
(\mathrm{~km})\end{array}$ & Location \\
\hline 2018-06-05 21:25 & 35141714 & 13.6 & 2.8 & 5.6 & 90 & 1074 & Australia, Tasmania \\
2018-08-12 22:56 & 36054296 & 13.4 & 0.1 & 11.5 & 80 & 847 & Australia \\
2018-11-16 21:00 & 34044155 & 12.0 & 0.1 & 16.1 & 86 & 990 & South Africa, La Réunion Island (FR) \\
2018-12-13 02:38 & 33690629 & 12.4 & 0.6 & 22.5 & 93 & 841 & Chile, Argentina, Brazil (South) \\
2020-01-04 17:16 & 34104468 & 12.2 & 0.1 & 23.4 & 79 & 896 & China, Japan \\
2020-01-21 13:53 & 34460788 & 11.9 & 0.1 & 17.1 & 88 & 880 & Australia, New Zealand (North) \\
2020-02-10 13:47 & 35021656 & 11.9 & 3.1 & 17.2 & 94 & 771 & Australia, New Zealand (South) \\
2020-02-13 23:50 & 35207286 & 12.0 & 0.1 & 17.9 & 96 & 1000 & Canada, Canary islands, Africa \\
\hline
\end{tabular}

\section{References}

A'Hearn, M.F., Feldman, P.D., 1992. Water vaporization on ceres. Icarus 98, 54-60. doi:10.1016/0019-1035(92)90206-M.

Assafin, M., Campos, R.P., Vieira Martins, R., da Silva Neto, D.N., Camargo, J.I.B., Andrei, A.H., 2008. Instrumental and digital coronagraphy for the observation of the uranus satellites' upcoming mutual events. Planet Space Sci. 56, 1882-1887. doi:10.1016/j.pss.2007.05.030.

Berthier, J., 1999. Principe de réduction des occultations stellaires. Notes scientifique et techniques du Bureau des longitudes S064.

Berthier, J., Hestroffer, D., Carry, B., Durech, J., Tanga, P., Delbo, M., Vachier, F., 2008. A service of position and physical ephemerides computation dedicated to the small bodies of the solar system. LPI Contributions 1405, 8374.

Berthier, J., Vachier, F., Marchis, F., Durech, J., Carry, B., 2014. Physical and dynamical properties of the main belt triple asteroid (87) sylvia. Icarus 239, 118-130. doi:10.1016/j.icarus.2014.05.046.

Beuzit, J.-L., Feldt, M., Dohlen, K., Mouillet, D., Puget, P., Wildi, F., Abe, L., Antichi, J., Baruffolo, A., Baudoz, P., Boccaletti, A., Carbillet, M., Charton, J., Claudi, R., Downing, M., Fabron, C., Feautrier, P., Fedrigo, E., Fusco, T., Gach, J.-L., Gratton, R., Henning, T., Hubin, N., Joos, F., Kasper, M., Langlois, M., Lenzen, R., Moutou, C., Pavlov, A., Petit, C., Pragt, J., Rabou, P., Rigal, F., Roelfsema, R., Rousset, G., Saisse, M., Schmid, H.-M., Stadler, E., Thalmann, C., Turatto, M., Udry, S., Vakili, F., Waters, R., 2008. SPHERE: a 'Planet Finder' instrument for the VLT. In: Ground-based and Airborne Instrumentation for Astronomy II. In: SPIE, 7014, p. 701418. doi:10.1117/12.790120.

Britt, D.T., Yeomans, D.K., Housen, K.R., Consolmagno, G.J., 2002. Asteroid density, porosity, and structure. Asteroids III 485-500.
Brownlee, D., Tsou, P., Aléon, J., Alexander, C.M.O., Araki, T., Bajt, S., Baratta, G.A., Bastien, R., Bland, P., Bleuet, P., Borg, J., Bradley, J.P., Brearley, A., Brenker, F., Brennan, S., Bridges, J.C., Browning, N.D., Brucato, J.R., Bullock, E., Burchell, M.J., Busemann, H., Butterworth, A., Chaussidon, M., Cheuvront, A., Chi, M., Cintala, M.J., Clark, B.C., Clemett, S.J., Cody, G., Colangeli, L., Cooper, G., Cordier, P. Daghlian, C., Dai, Z., D'Hendecourt, L., Djouadi, Z., Dominguez, G., Duxbury, T., Dworkin, J.P., Ebel, D.S., Economou, T.E., Fakra, S., Fairey, S.A.J., Fallon, S., Ferrini, G., Ferroir, T., Fleckenstein, H., Floss, C., Flynn, G., Franchi, I.A., Fries, M., Gainsforth, Z., Gallien, J.-P., Genge, M., Gilles, M.K., Gillet, P., Gilmour, J., Glavin, D.P., Gounelle, M., Grady, M.M., Graham, G.A., Grant, P.G., Green, S.F. Grossemy, F., Grossman, L., Grossman, J.N., Guan, Y., Hagiya, K., Harvey, R., Heck, P., Herzog, G.F., Hoppe, P., Hörz, F., Huth, J., Hutcheon, I.D., Ignatyev, K., Ishii, H., Ito, M., Jacob, D., Jacobsen, C., Jacobsen, S., Jones, S., Joswiak, D., Jurewicz, A., Kearsley, A.T., Keller, L.P., Khodja, H., Kilcoyne, A.L.D., Kissel, J., Krot, A., Langenhorst, F., Lanzirotti, A., Le, L., Leshin, L.A., Leitner, J., Lemelle, L., Leroux, H., Liu, M.-C., Luening, K., Lyon, I., MacPherson, G., Marcus, M.A., Marhas, K., Marty, B., Matrajt, G., McKeegan, K., Meibom, A., Mennella, V., Messenger, K., Messenger, S., Mikouchi, T., Mostefaoui, S., Nakamura, T., Nakano, T., Newville, M., Nittler, L.R., Ohnishi, I., Ohsumi, K., Okudaira, K., Papanastassiou, D.A., Palma, R., Palumbo, M.E., Pepin, R.O., Perkins, D., Perronnet, M., Pianetta, P., Rao, W., Rietmeijer, F.J.M., Robert, F., Rost, D., Rotundi, A., Ryan, R. Sandford, S.A., Schwandt, C.S., See, T.H., Schlutter, D., Sheffield-Parker, J., Simionovici, A., Simon, S., Sitnitsky, I., Snead, C.J., Spencer, M.K., Stadermann, F.J., Steele, A., Stephan, T., Stroud, R., Susini, J., Sutton, S.R., Suzuki, Y., Taheri, M., Taylor, S., Teslich, N., Tomeoka, K., Tomioka, N., Toppani, A., Trigo-Rodríguez, J.M., 
Troadec, D., Tsuchiyama, A., Tuzzolino, A.J., Tyliszczak, T., Uesugi, K., Velbel, M., Vellenga, J., Vicenzi, E., Vincze, L., Warren, J., Weber, I., Weisberg, M., Westphal, A.J., Wirick, S., Wooden, D., Wopenka, B., Wozniakiewicz, P., Wright, I., Yabuta, H., Yano, H., Young, E.D., Zare, R.N., Zega, T., Ziegler, K., Zimmerman, L., Zinner, E., Zolensky, M., 2006. Comet 81p/wild 2 under a microscope. Science 314, 1711. doi:10.1126/science. 1135840.

Buie, M.W., Olkin, C.B., Merline, W.J., Walsh, K.J., Levison, H.F., Timerson, B., Herald, D., Owen Jr., W.M., Abramson, H.B., Abramson, K.J., Breit, D.C., Caton, D.B., Conard, S.J., Croom, M.A., Dunford, R.W., Dunford, J.A., Dunham, D.W., Ellington, C.K., Liu, Y., Maley, P.D., Olsen, A.M., Preston, S., Royer, R., Scheck, A.E., Sherrod, C., Sherrod, L., Swift, T.J., Taylor III, L.W., Venable, R., 2015. Size and shape from stellar occultation observations of the double jupiter trojan patroclus and menoetius. Astron. J. (NY) 149, 113. doi:10.1088/0004-6256/149/3/113.

Burbine, T.H., McCoy, T.J., Meibom, A., Gladman, B., Keil, K., 2002. Meteoritic parent bodies: their number and identification. Asteroids III 653-667.

Burbine, T.H., Meibom, A., Binzel, R.P., 1996. Mantle material in the main belt: battered to bits? Meteoritics Planet. Sci. 31, 607-620.

Bus, S.J., Binzel, R.P., 2002a. Phase II of the small main-Belt asteroid spectroscopic survey: a feature-based taxonomy. Icarus 158, 146-177. doi:10.1006/icar.2002. 6856.

Bus, S.J., Binzel, R.P., 2002b. Phase II of the small main-belt asteroid spectroscopic survey: the observations. Icarus 158, 106-145. doi:10.1006/icar.2002.6857.

Campins, H., Hargrove, K., Pinilla-Alonso, N., Howell, E.S., Kelley, M.S., Licandro, J., Mothé-Diniz, T., Fernández, Y., Ziffer, J., 2010. Water ice and organics on the surface of the asteroid 24 themis. Nature 464, 1320-1321. doi:10.1038/ nature09029.

Carry, B., 2012. Density of asteroids. Planet Space Sci. 73, 98-118. doi:10.1016/j.pss. 2012.03.009.

Carry, B., Dumas, C., Fulchignoni, M., Merline, W.J., Berthier, J., Hestroffer, D., Fusco, T., Tamblyn, P., 2008. Near-Infrared mapping and physical properties of the dwarf-planet ceres. Astron. Astrophys. 478 (4), 235-244. doi:10.1051/ 0004-6361:20078166.

Carry, B., Dumas, C., Kaasalainen, M., Berthier, J., Merline, W.J., Erard, S., Conrad, A.R., Drummond, J.D., Hestroffer, D., Fulchignoni, M., Fusco, T., 2010a. Physical properties of (2) pallas. Icarus 205, 460-472. doi:10.1016/j.icarus.2009.08.007.

Carry, B., Hestroffer, D., DeMeo, F.E., Thirouin, A., Berthier, J., Lacerda, P., Sicardy, B., Doressoundiram, A., Dumas, C., Farrelly, D., Müller, T.G., 2011. Integral-field spectroscopy of (90482) orcus-Vanth. Astron. Astrophys. 534, A115. doi:10.1051/ 0004-6361/201117486.

Carry, B., Kaasalainen, M., Leyrat, C., Merline, W.J., Drummond, J.D., Conrad, A.R., Weaver, H.A., Tamblyn, P.M., Chapman, C.R., Dumas, C., Colas, F., Christou, J.C., Dotto, E., Perna, D., Fornasier, S., Bernasconi, L., Behrend, R., Vachier, F., Kryszczynska, A., Polinska, M., Fulchignoni, M., Roy, R., Naves, R., Poncy, R., Wiggins, P., 2010b. Physical properties of the ESA rosetta target asteroid (21) lutetia. II. shape and flyby geometry. Astron. Astrophys. 523, A94. doi:10.1051/ 0004-6361/201015074.

Carry, B., Kaasalainen, M., Merline, W.J., Müller, T.G., Jorda, L., Drummond, J.D., Berthier, J., O'Rourke, L., Ďurech, J., Küppers, M., Conrad, A.R., Dumas, C., Sierks, H., The OSIRIS TeamPSS, 2012. Koala shape modeling technique validated at (21) lutetia by esa rosetta mission. Planet Space Sci. 66, 200-212. doi:10.1016/j.pss.2011.12.018.

Carry, B., Matter, A., Scheirich, P., Pravec, P., Molnar, L., Mottola, S., Carbognani, A., Jehin, E., Marciniak, A., Binzel, R.P., DeMeo, F.E., Birlan, M., Delbo, M., Barbotin, E., Behrend, R., Bonnardeau, M., Colas, F., Farissier, P., Fauvaud, M., Fauvaud, S., Gillier, C., Gillon, M., Hellmich, S., Hirsch, R., Leroy, A., Manfroid, J., Montier, J., Morelle, E., Richard, F., Sobkowiak, K., Strajnic, J., Vachier, F., 2015. The small binary asteroid (939) isberga. Icarus 248, 516-525. doi:10.1016/j.icarus.2014.11. 002 .

Claudi, R.U., Turatto, M., Gratton, R.G., Antichi, J., Bonavita, M., Bruno, P., Cascone, E., De Caprio, V., Desidera, S., Giro, E., Mesa, D., Scuderi, S., Dohlen, K., Beuzit, J.L., Puget, P., 2008. SPHERE IFS: the spectro differential imager of the VLT for exoplanets search. In: Ground-based and Airborne Instrumentation for Astronomy II. In: SPIE, 7014, p. 70143E. doi:10.1117/12.788366.

Combe, J.-P., McCord, T.B., Tosi, F., Ammannito, E., Carrozzo, F.G., De Sanctis, M.C. Raponi, A., Byrne, S., Landis, M.E., Hughson, K.H.G., Raymond, C.A., Russell, C.T. 2016. Detection of local $h_{2} \mathrm{o}$ exposed at the surface of ceres. Science 353 aaf3010. doi:10.1126/science.aaf3010.

Cushing, M.C., Vacca, W.D., Rayner, J.T., 2004. Spextool: a spectral extraction package for SpeX, a 0.8-5.5 micron cross-Dispersed spectrograph. Publ. Astron. Soc. Pac. 116, 362-376. doi:10.1086/382907.

van Dam, M.A., Le Mignant, D., Macintosh, B., 2004. Performance of the Keck observatory adaptive-optics system. Appl. Opt. 43 (23), 5458-5467.

Delbo, M., Tanga, P., Carry, B., Ordenovic, C., Bottein, P., 2017. $\mathrm{Mp}^{3} \mathrm{c}$ : The Minor Planet Physical Properties catalogue. ACM.

DeMeo, F.E., Binzel, R.P., Slivan, S.M., Bus, S.J., 2009. An extension of the bus asteroid taxonomy into the near-infrared. Icarus 202, 160-180. doi:10.1016/j.icarus.2009. 02.005

DeMeo, F.E., Carry, B., 2014. Solar system evolution from compositional mapping of the asteroid belt. Nature 505, 629-634. doi:10.1038/nature12908.

DeMeo, F.E., Carry, B., Marchis, F., Birlan, M., Binzel, R.P., Bus, S.J., Descamps, P., Nedelcu, A., Busch, M., Bouy, H., 2011. A spectral comparison of (379) huenna and its satellite. Icarus 212, 677-681. doi:10.1016/j.icarus.2011.02.002.

Dobrovolskis, A.R., 1996. Inertia of any polyhedron. Icarus 124, 698-704. doi:10. 1006/icar:1996.0243.

Dohlen, K., Langlois, M., Saisse, M., Hill, L., Origne, A., Jacquet, M., Fabron, C., Blanc, J.-C., Llored, M., Carle, M., Moutou, C., Vigan, A., Boccaletti, A., Carbil- let, M., Mouillet, D., Beuzit, J.-L., 2008. The infra-red dual imaging and spectrograph for SPHERE: design and performance. In: Ground-based and Airborne Instrumentation for Astronomy II. In: SPIE, 7014, p. 70143L. doi:10.1117/12.789786.

Drummond, J.D., Conrad, A., Merline, W.J., Carry, B., Chapman, C.R., Weaver, H.A., Tamblyn, P.M., Christou, J.C., Dumas, C., 2010. Physical properties of the ESA rosetta target asteroid (21) Lutetia. i. The triaxial ellipsoid dimensions, rotational pole, and bulk density. Astron. Astrophys. 523, A93. doi:10.1051/0004-6361/ 201015075.

Drummond, J.D., Reynolds, O.R., Buckman, M.D., 2016. The orbit and size of (87) Sylvia's Romulus from the 2015 apparition. Icarus 276, 107-115. doi:10.1016/j. icarus.2016.04.033.

Dunham, D., Herald, D., preston, S., Timerson, B., Maley, P., Frappa, E., Hayamizu, T., Talbot, J., Poro, A., 2016. Sizes, shapes, and satellites of asteroids from occultations. In: Chesley, S.R., Morbidelli, A., Jedicke, R., Farnocchia, D. (Eds.), Asteroids: New Observations, New Models, pp. 177-180

Dunham, D. W., Herald, D., Frappa, E., Hayamizu, T., Talbot, J., Timerson, B. 2016b. Asteroid occultations. NASA Planetary Data System. EAR-A-3-RDROCCULTATIONS-V14.0.

Ďurech, J., Kaasalainen, M., Herald, D., Dunham, D., Timerson, B., Hanuš, J., Frappa, E., Talbot, J., Hayamizu, T., Warner, B.D., Pilcher, F., Galád, A., 2011. Combining asteroid models derived by lightcurve inversion with asteroidal occultation silhouettes. Icarus 214, 652-670. doi:10.1016/j.icarus.2011.03.016.

Ďurech, J., Sidorin, V., Kaasalainen, M., 2010. DAMIT: A database of asteroid models. Astron. Astrophys. 513, A46. doi:10.1051/0004-6361/200912693.

Emery, J.P., Cruikshank, D.P., van Cleve, J., 2006. Thermal emission spectroscopy (5.2 $38 \mu \mathrm{m}$ ) of three trojan asteroids with the spitzer space telescope: detection of fine-grained silicates. Icarus 182, 496-512. doi:10.1016/j.icarus.2006.01.011.

Fang, J., Margot, J.-L., Rojo, P., 2012. Orbits, masses, and evolution of main belt triple (87) sylvia. Astron. J. 144, 70. doi:10.1088/0004-6256/144/2/70.

Fienga, A., Kuchynka, P., Laskar, J., Manche, H., Gastineau, M., 2011. Asteroid mass determinations with INPOP planetary ephemerides. In: Proceedings of the EPSC-DPS Joint Meeting 2011, p. 1879.

Fienga, A., Manche, H., Kuchynka, P., Laskar, J., Gastineau, M., 2010. INPOP (Integration Numerique Planetaire de l'Observatoire de Paris), Planetary and Lunar ephemerides. INPOP10A.

Fienga, A., Manche, H., Laskar, J., Gastineau, M., Verma, A., 2013. New planetary ephemerides based on MESSENGER tracking. Documentation for the INPOP10e release. Scientific notes. ArXiv e-print 1301.1510.

Fusco, T., 2000. Correction Partielle Et Anisoplanétisme En Optique. Université de Nice Sophia-Antipolis Ph.D. thesis,

Fusco, T., Rousset, G., Sauvage, J.-F., Petit, C., Beuzit, J.-L., Dohlen, K., Mouillet, D. Charton, J., Nicolle, M., Kasper, M., Baudoz, P., Puget, P., 2006. High-order adaptive optics requirements for direct detection of extrasolar planets: application to the SPHERE instrument. Opt. Express 14, 7515. doi:10.1364/OE.14.007515.

Gaia Collaboration, Prusti, T., de Bruijne, J.H.J., Brown, A.G.A., Vallenari, A., Babusiaux, C., Bailer-Jones, C.A.L., Bastian, U., Biermann, M., Evans, D.W., et al., 2016. The Gaia mission. Astron. Astrophys. 595, A1. doi:10.1051/0004-6361/ 201629272.

Goffin, E., 2014. Astrometric asteroid masses: a simultaneous determination. Astron Astrophys. 565, A56. doi:10.1051/0004-6361/201322766.

Gradie, J., Tedesco, E., 1982. Compositional structure of the asteroid belt. Science 216, 1405-1407. doi:10.1126/science.216.4553.1405.

Greenberg, J.M., 2000. From comets to meteors. Earth Moon Planets 82, 313-324.

Grice, J., Snodgrass, C., Green, S., Parley, N., Carry, B., 2017. The shapes of primordial asteroids. In: Proceedings of the Asteroids, Comets, and Meteors: ACM 2017.

Gwyn, S.D.J., Hill, N., Kavelaars, J.J., 2012. Ssos: a moving-object image search tool for asteroid precovery. Publ. Astron. Soc. Pac. 124, 579. doi:10.1086/666462.

Hanuš, J., Marchis, F., Ďurech, J., 2013. Sizes of main-belt asteroids by combining shape models and Keck adaptive optics observations. Icarus 226, 1045-1057. doi:10.1016/j.icarus.2013.07.023.

Hanuš, J., Durech, J., Oszkiewicz, D.A., Behrend, R., Carry, B., Delbo, M., Adam, O., Afonina, V., Anquetin, R., Antonini, P., Arnold, L., Audejean, M., Aurard, P., Bachschmidt, M., Baduel, B., Barbotin, E., Barroy, P., Baudouin, P., Berard, L., Berger, N. Bernasconi, L., Bosch, J.-G., Bouley, S., Bozhinova, I., Brinsfield, J., Brunetto, L., Canaud, G., Caron, J., Carrier, F., Casalnuovo, G., Casulli, S., Cerda, M., Chalamet, L., Charbonnel, S., Chinaglia, B., Cikota, A., Colas, F., Coliac, J.-F., Collet, A., Coloma, J., Conjat, M., Conseil, E., Costa, R., Crippa, R., Cristofanelli, M. Damerdji, Y., Debackère, A., Decock, A., Déhais, Q., Déléage, T., Delmelle, S., Demeautis, C., Dróżdż, M., Dubos, G., Dulcamara, T., Dumont, M., Durkee, R., Dymock, R., Escalante del Valle, A., Esseiva, N., Esseiva, R., Esteban, M. Fauchez, T., Fauerbach, M., Fauvaud, M., Fauvaud, S., Forné, E., Fournel, C., Fradet, D., Garlitz, J., Gerteis, O., Gillier, C., Gillon, M., Giraud, R., Godard, J.P., Goncalves, R., Hamanowa, H., Hamanowa, H., Hay, K., Hellmich, S., Heterier, S., Higgins, D., Hirsch, R., Hodosan, G., Hren, M., Hygate, A., Innocent, N., Jacquinot, H., Jawahar, S., Jehin, E., Jerosimic, L., Klotz, A., Koff, W., Korlevic, P., Kosturkiewicz, E., Krafft, P., Krugly, Y., Kugel, F. Labrevoir, O., Lecacheux, J., Lehký, M. Leroy, A., Lesquerbault, B., Lopez-Gonzales, M.J., Lutz, M., Mallecot, B. Manfroid, J., Manzini, F., Marciniak, A., Martin, A., Modave, B., Montaigut, R., Montier, J., Morelle, E., Morton, B., Mottola, S., Naves, R., Nomen, J., Oey, J., Ogłoza, W., Paiella, M., Pallares, H., Peyrot, A., Pilcher, F., Pirenne, J.-F., Piron, P., Polińska, M., Polotto, M., Poncy, R., Previt, J.P., Reignier, F., Renauld, D., Ricci, D. Richard, F., Rinner, C., Risoldi, V., Robilliard, D., Romeuf, D., Rousseau, G., Roy, R., Ruthroff, J., Salom, P.A., Salvador, L., Sanchez, S., Santana-Ros, T., Scholz, A., Séné, G., Skiff, B., Sobkowiak, K., Sogorb, P., Soldán, F., Spiridakis, A., Splanska, E., Sposetti, S., Starkey, D., Stephens, R., Stiepen, A., Stoss, R., Strajnic, J., Teng, J.-P., Tumolo, G., Vagnozzi, A., Vanoutryve, B., Vugnon, J.M., Warner, B.D. 
Waucomont, M., Wertz, O., Winiarski, M., Wolf, M., 2016. New and updated convex shape models of asteroids based on optical data from a large collaboration network. Astron. Astrophys. 586, A108. doi:10.1051/0004-6361/201527441.

Hanuš, J., Viikinkoski, M., Marchis, F., Ďurech, J., Kaasalainen, M., Delbo', M., Herald, D., Frappa, E., Hayamizu, T., Kerr, S., Preston, S., Timerson, B., Dunham, D., Talbot, J., 2017. Volumes and bulk densities of forty asteroids from ADAM shape modeling. Astron. Astrophys. 601, A114. doi:10.1051/0004-6361/201629956.

Harris, A.W., Young, J.W., 1989. Asteroid lightcurve observations from 1979 to 1981. Icarus 81, 314-364. doi:10.1016/0019-1035(89)90056-0.

Herriot, G., Morris, S., Anthony, A., Derdall, D., Duncan, D., Dunn, J., Ebbers, A.W., Fletcher, J.M., Hardy, T., Leckie, B., Mirza, A., Morbey, C.L., Pfleger, M., Roberts, S., Shott, P., Smith, M., Saddlemyer, L.K., Sebesta, J., Szeto, K., Wooff, R., Windels, W., Veran, J.-P., 2000. Progress on Altair: the Gemini North adaptive optics system. In: Wizinowich, P.L. (Ed.), Adaptive Optical Systems Technology. In: Society of Photo-Optical Instrumentation Engineers (SPIE) Conference Series, 4007, pp. 115-125

Hodapp, K.W., Jensen, J.B., Irwin, E.M., Yamada, H., Chung, R., Fletcher, K., Robertson, L., Hora, J.L., Simons, D.A., Mays, W., Nolan, R., Bec, M., Merrill, M. Fowler, A.M., 2003. The gemini near-Infrared imager (NIRI). Publ. Astron. Soc. Pac. 115, 1388-1406. doi:10.1086/379669.

Kaasalainen, M., 2011. Maximum compatibility estimates and shape reconstruction with boundary curves and volumes of generalized projections. Inverse Probl. Imag. 5 (1), 37-57.

Kaasalainen, M., Viikinkoski, M., 2012. Shape reconstruction of irregular bodies with multiple complementary data sources. Astron. Astrophys. 543, A97. doi:10.1051/ 0004-6361/201219267.

Küppers, M., O'Rourke, L., Bockelée-Morvan, D., Zakharov, V., Lee, S., von Allmen, P., Carry, B., Teyssier, D., Marston, A., Müller, T., Crovisier, J., Barucci, M.A., Moreno, R., 2014. Localized sources of water vapour on the dwarf planet (1)ceres. Nature 505, 525-527. doi:10.1038/nature12918.

Lagerkvist, C.-I., Magnusson, P., 2011. Asteroid Photometric Catalog V1.1. EAR-A-3DDR-APC-LIGHTCURVE-V1.1. NASA Planetary Data System.

Laver, C., de Pater, I., Marchis, F., Ádámkovics, M., Wong, M.H., 2009. Componentresolved near-infrared spectra of the (22) kalliope system. Icarus 204, 574-579. doi:10.1016/j.icarus.2009.07.002

Lazzaro, D., Angeli, C.A., Carvano, J.M., Mothé-Diniz, T., Duffard, R., Florczak, M., 2004. $S^{3}$ OS $^{2}$ : the visible spectroscopic survey of 820 asteroids. Icarus 172, 179220. doi:10.1016/j.icarus.2004.06.006.

Lenzen, R., Hartung, M., Brandner, W., Finger, G., Hubin, N.N., Lacombe, F., Lagrange, A.-M., Lehnert, M.D., Moorwood, A.F.M., Mouillet, D., 2003. NAOS-CONICA First on sky results in a variety of observing modes. SPIE 4841, 944-952.

Licandro, J., Campins, H., Kelley, M., Hargrove, K., Pinilla-Alonso, N., Cruikshank, D. Rivkin, A.S., Emery, J., 2011. (65) Cybele: detection of small silicate grains, water-ice, and organics. Astron. Astrophys. 525, A34. doi:10.1051/0004-6361/ 201015339.

Lindsay, S.S., Marchis, F., Emery, J.P., Enriquez, J.E., Assafin, M., 2015. Composition, mineralogy, and porosity of multiple asteroid systems from visible and nearinfrared spectral data. Icarus 247, 53-70. doi:10.1016/j.icarus.2014.08.040.

Lundborg, N., 1967. The strength-size relation of granite 4, 269-272.

Marchis, F., Descamps, P., Baek, M., Harris, A.W., Kaasalainen, M., Berthier, J., Hestroffer, D., Vachier, F., 2008. Main belt binary asteroidal systems with circular mutual orbits. Icarus 196 (1), 97-118. doi:10.1016/j.icarus.2008.03.007.

Marchis, F., Descamps, P., Berthier, J., Hestroffer, D., Vachier, F., Baek, M., Harris, A.W. Nesvorný, D., 2008. Main belt binary asteroidal systems with eccentric mutual orbits. Icarus 195 (1), 295-316. doi:10.1016/j.icarus.2007.12.010.

Marchis, F., Durech, J., Castillo-Rogez, J., Vachier, F., Cuk, M., Berthier, J., Wong, M.H. Kalas, P., Duchene, G., van Dam, M.A., Hamanowa, H., Viikinkoski, M., 2014. The puzzling mutual orbit of the binary trojan asteroid (624) hektor. Astrophys. J. 783, L37. doi:10.1088/2041-8205/783/2/L37.

Marchis, F., Enriquez, J.E., Emery, J.P., Berthier, J., Descamps, P., Vachier, F., 2011. The origin of (90) antiope from component-resolved near-infrared spectroscopy. Icarus 213, 252-264. doi:10.1016/j.icarus.2011.02.011.

Marchis, F., Enriquez, J.E., Emery, J.P., Mueller, M., Baek, M., Pollock, J., Assafin, M., Vieira Martins, R., Berthier, J., Vachier, F., Cruikshank, D.P., Lim, L.F., Reichart, D.E., Ivarsen, K.M., Haislip, J.B., LaCluyze, A.P., 2012. Multiple asteroid systems: dimensions and thermal properties from spitzer space telescope and ground-based observations. Icarus 221, 1130-1161. doi:10.1016/j.icarus.2012.09. 013.

Marchis, F., Kaasalainen, M., Hom, E.F.Y., Berthier, J., Enriquez, J., Hestroffer, D., Le Mignant, D., de Pater, I., 2006. Shape, size and multiplicity of main-belt asteroids. Icarus 185 (1), 39-63.

Marchis, F., Lainey, V., Descamps, P., Berthier, J., Van Dam, M., de Pater, I., Macomber, B., Baek, M., Le Mignant, D., Hammel, H.B., Showalter, M., Vachier, F., 2010. A dynamical solution of the triple asteroid system (45) eugenia. Icarus 210, 635-643. doi:10.1016/j.icarus.2010.08.005.

Margot, J.-L., Pravec, P., Taylor, P., Carry, B., Jacobson, S., 2015. Asteroid Systems: Binaries, Triples, and Pairs. University of Arizona Press, pp. 355-374. doi:10.2458/ azu_uapress_9780816532131-ch019.

Marsset, M., Carry, B., Dumas, C., Hanuš, J., Viikinkoski, M., Vernazza, P., Müller, T.G., Delbo, M., Jehin, E., Gillon, M., Grice, J., Yang, B., Fusco, T., Berthier, J., Sonnett, S., Kugel, F., Caron, J., Behrend, R., 2017. 3D Shape of asteroid (6) hebe from VLT/SPHERE imaging: implications for the origin of ordinary h chondrites. Astron. Astrophys. 604, A64. doi:10.1051/0004-6361/201731021.

Marsset, M., Carry, B., Yang, B., Marchis, F., Vernazza, P., Dumas, C., Berthier, J. Vachier, F., 2016. S/2016 (107) 1. IAU Circular 9282. di Martino, M., Zappala, V., de Sanctis, G., Cacciatori, S., 1987. Photoelectric photometry of 17 asteroids. Icarus 69, 338-353. doi:10.1016/0019-1035(87)90110-2.

Masiero, J.R., Mainzer, A.K., Grav, T., Bauer, J.M., Cutri, R.M., Dailey, J., Eisenhardt, P.R.M., McMillan, R.S., Spahr, T.B., Skrutskie, M.F., Tholen, D., Walker, R.G. Wright, E.L., DeBaun, E., Elsbury, D., Gautier IV, T., Gomillion, S., Wilkins, A., 2011. Main belt asteroids with WISE/NEOWISE. i. preliminary albedos and diameters. Astrophys. J. 741, 68. doi:10.1088/0004-637X/741/2/68.

Merline, W.J., Close, L.M., Dumas, C., Chapman, C.R., Roddier, F., Ménard, F. Slater, D.C., Duvert, G., Shelton, C., Morgan, T., 1999. Discovery of a moon orbiting the asteroid 45 eugenia. Nature 401, 565-568.

Merline, W.J., Weidenschilling, S.J., Durda, D.D., Margot, J.-L., Pravec, P., Storrs, A.D., 2002. Asteroids do have satellites. Asteroids III 289-312.

Morbidelli, A., Bottke, W.F., Nesvorný, D., Levison, H.F., 2009. Asteroids were born big. Icarus 204, 558-573. doi:10.1016/j.icarus.2009.07.011

Morrison, D., Zellner, B., 2007. TRIAD Radiometric Diameters and Albedos. NASA Planetary Data System. EAR-A-COMPIL-5-TRIADRAD-V1.0.

Mousis, O., Hueso, R., Beaulieu, J.-P., Bouley, S., Carry, B., Colas, F., Klotz, A., Pellier, C., Petit, J.-M., Rousselot, P., Ali-Dib, M., Beisker, W., Birlan, M., Buil, C., Delsanti, A., Frappa, E., Hammel, H.B., Levasseur-Regourd, A.C., Orton, G.S. Sánchez-Lavega, A., Santerne, A., Tanga, P., Vaubaillon, J., Zanda, B., Baratoux, D., Böhm, T., Boudon, V., Bouquet, A., Buzzi, L., Dauvergne, J.-L., Decock, A., Delcroix, M., Drossart, P., Esseiva, N., Fischer, G., Fletcher, L.N., Foglia, S., GómezForrellad, J.M., Guarro-Fló, J., Herald, D., Jehin, E., Kugel, F., Lebreton, J.-P., Lecacheux, J., Leroy, A., Maquet, L., Masi, G., Maury, A., Meyer, F., Pérez-Hoyos, S. Rajpurohit, A.S., Rinner, C., Rogers, J.H., Roques, F., Schmude, R.W., Sicardy, B., Tregon, B., Vanhuysse, M., Wesley, A., Widemann, T., 2014. Instrumental methods for professional and amateur collaborations in planetary astronomy. Exp. Astron. 38, 91-191. doi:10.1007/s10686-014-9379-0.

Mueller, M., Marchis, F., Emery, J.P., Harris, A.W., Mottola, S., Hestroffer, D., Berthier, J., di Martino, M., 2010. Eclipsing binary trojan asteroid patroclus: thermal inertia from spitzer observations. Icarus 205, 505-515. doi:10.1016/j.icarus. 2009.07.043.

Mugnier, L.M., Fusco, T., Conan, J.-M., 2004. MISTRAL: A myopic edge-Preserving image restoration method, with application to astronomical adaptive-Optics-Corrected long-Exposure images. J. Opt. Soc. Am. A 21 (10), 1841-1854.

Nedelcu, D.A., Birlan, M., Vernazza, P., Descamps, P., Binzel, R.P., Colas, F., Kryszczynska, A., Bus, S.J., 2007. Near infra-red spectroscopy of the asteroid 21 Lutetia. Il. Rotationally resolved spectroscopy of the surface. Astron. Astrophys. 470, 11571164. doi:10.1051/0004-6361:20066944.

Parley, N.R., McBride, N., Green, S.F., Haswell, C.A., Clarkson, W.I., Christian, D.J., Collier-Cameron, A., Evans, N.A., Fitzsimmons, A., Hellier, C., Hodgkin, S.T., Horne, K., Irwin, J., Kane, S.R., Keenan, F.P., Lister, T.A., Norton, A.J., Osborne, J.P. Pollacco, D., Ryans, R., Skillen, I., Street, R.A., West, R.G., Wheatley, P.J., 2005. Serendipitous asteroid lightcurve survey using superwasp. Earth Moon Planets 97, 261-268. doi:10.1007/s11038-006-9072-z.

Pätzold, M., Andert, T., Hahn, M., Asmar, S.W., Barriot, J.-P., Bird, M.K., Häusler, B. Peter, K., Tellmann, S., Grün, E., Weissman, P.R., Sierks, H., Jorda, L., Gaskell, R., Preusker, F., Scholten, F., 2016. A homogeneous nucleus for comet 67p/Churyumov-Gerasimenko from its gravity field. Nature 530, 63-65. doi:10. 1038/nature16535.

Pavlov, A., Möller-Nilsson, O., Feldt, M., Henning, T., Beuzit, J.-L., Mouillet, D., 2008 SPHERE data reduction and handling system: overview, project status, and development. In: Advanced Software and Control for Astronomy II. In: SPIE, 7019, p. 701939. doi:10.1117/12.789110.

Polishook, D., 2009. Lightcurves for shape modeling obtained at the wise observatory. Minor Planet Bull. 36, 119-120.

Polishook, D., Brosch, N., Prialnik, D., Kaspi, S., 2009. Simultaneous spectroscopic and photometric observations of binary asteroids. Meteoritics Planet. Sci. 44 1955-1966. doi:10.1111/j.1945-5100.2009.tb02005.x.

Pollacco, D.L., Skillen, I., Collier Cameron, A., Christian, D.J., Hellier, C., Irwin, J., Lister, T.A., Street, R.A., West, R.G., Anderson, D.R., Clarkson, W.I., Deeg, H., Enoch, B., Evans, A., Fitzsimmons, A., Haswell, C.A., Hodgkin, S., Horne, K. Kane, S.R., Keenan, F.P., Maxted, P.F.L., Norton, A.J., Osborne, J., Parley, N.R., Ryans, R.S.I., Smalley, B., Wheatley, P.J., Wilson, D.M., 2006. The WASP project and the superwasp cameras. Publ. Astron. Soc. Pac. 118 (848), 1407-1418.

Popescu, M., Birlan, M., Nedelcu, D.A., 2012. Modeling of asteroid spectra - M4AST. Astron. Astrophys. 544, A130. doi:10.1051/0004-6361/201219584.

Rayner, J.T., Toomey, D.W., Onaka, P.M., Denault, A.J., Stahlberger, W.E., Vacca, W.D. Cushing, M.C., Wang, S., 2003. Spex: A Medium-Resolution 0.8-5.5 micron spectrograph and imager for the NASA infrared telescope facility. Publ. Astron. Soc Pac. 115, 362-382. doi:10.1086/367745

Rivkin, A.S., Emery, J.P., 2010. Detection of ice and organics on an asteroidal surface Nature 464, 1322-1323. doi:10.1038/nature09028.

Rivkin, A.S., Howell, E.S., Vilas, F., Lebofsky, L.A., 2002. Hydrated minerals on asteroids: the astronomical record. Asteroids III 235-253.

Rivkin, A.S., Volquardsen, E.L., Clark, B.E., 2006. The surface composition of ceres: discovery of carbonates and iron-rich clays. Icarus 185, 563-567. doi:10.1016/j. icarus.2006.08.022.

Rousselot, P., Mousis, O., Dumas, C., Jehin, E., Manfroid, J., Carry, B., Zucconi, J.-M., 2011. A search for water vaporization on ceres. Astron. J. 142, A125.

Rousset, G., Lacombe, F., Puget, P., Hubin, N.N., Gendron, E., Fusco, T., Arsenault, R. Charton, J., Feautrier, P., Gigan, P., Kern, P.Y., Lagrange, A.-M., Madec, P.-Y., Mouillet, D., Rabaud, D., Rabou, P., Stadler, E., Zins, G., 2003. NAOS, The first AO system of the VLT: on-sky performance. SPIE 4839, 140-149.

Ryan, E.L., Woodward, C.E., 2010. Rectified asteroid albedos and diameters from IRAS 
and MSX photometry catalogs. Astron. J. 140, 933-943. doi:10.1088/0004-6256/ $140 / 4 / 933$.

Scheeres, D.J., Britt, D., Carry, B., Holsapple, K.A., 2015. Asteroid Interiors and Morphology. University of Arizona Press, pp. 745-766. doi:10.2458/azu_uapress_ 9780816532131-ch038.

Scheirich, P., Pravec, P., 2009. Modeling of lightcurves of binary asteroids. Icarus 200, 531-547. doi:10.1016/j.icarus.2008.12.001.

Sierks, H., Lamy, P., Barbieri, C., Koschny, D., Rickman, H., Rodrigo, R., A'Hearn, M.F., Angrilli, F., Barucci, A., Bertaux, J.-L., Bertini, I., Besse, S., Carry, B., Cremonese, G., Da Deppo, V., Davidsson, B., Debei, S., De Cecco, M., De Leon, J., Ferri, F., Fornasier, S., Fulle, M., Hviid, S.F., Gaskell, G.W., Groussin, O., Gutierrez, P.J., Jorda, L., Kaasalainen, M., Keller, H.U., Knollenberg, J., Kramm, J.R., Kührt, E., Küppers, M., Lara, L.M., Lazzarin, M., Leyrat, C., Lopez Moreno, J.L., Magrin, S., Marchi, S., Marzari, F., Massironi, M., Michalik, H., Moissl, R., Naletto, G., Preusker, F., Sabau, L., Sabolo, W., Scholten, F., Snodgrass, C., Thomas, N., Tubiana, C., Vernazza, P., Vincent, J.-B., Wenzel, K.-P., Andert, T., Pätzold, M., Weiss, B.P., 2011. Images of asteroid (21) Lutetia: a remnant planetesimal from the early solar system. Science 334, 487-490.

Spoto, F., Tanga, P., Bouquillon, S., Desmars, J., Hestroffer, D., Mignard, F., Altmann, M., Herald, D., Marchant, J., Barache, C., Carlucci, T., Lister, T., Taris, F., 2017. Ground-based astrometry calibrated by gaia DR1: new perspectives in asteroid orbit determination. Astron. Astrophys. 607, A21. doi:10.1051/0004-6361/ 201731439.

Storrs, A., Vilas, F., Landis, R., Wells, E., Woods, C., Zellner, B., Gaffey, M., 2001. S/2001 (107) 1. IAU Circular 7599, 3.

Takir, D., Emery, J.P., 2012. Outer main belt asteroids: identification and distribution of four 3- $\mu \mathrm{m}$ spectral groups. Icarus 219, 641-654. doi:10.1016/j.icarus.2012.02. 022.

Tanga, P., Delbo, M., 2007. Asteroid occultations today and tomorrow: toward the GAIA era. Astron. Astrophys. 474, 1015-1022. doi:10.1051/0004-6361:20077470.

Taylor, M.B., 2005. TOPCAT \& STIL: Starlink Table/VOTable Processing Software. In: Shopbell, P., Britton, M., Ebert, R. (Eds.), Astronomical Data Analysis Software and Systems XIV. In: Astronomical Society of the Pacific Conference Series, 347, p. 29 .

Tedesco, E.F., Noah, P.V., Noah, M.C., Price, S.D., 2002. The supplemental IRAS minor planet survey. Astron. J. 123, 1056-1085. doi:10.1086/338320.

Tedesco, E. F., Noah, P. V., Noah, M. C., Price, S. D., 2004. IRAS Minor Planet Survey. NASA Planetary Data System. IRAS-A-FPA-3-RDR-IMPS-V6.0.

Tedesco, E.F., Williams, J.G., Matson, D.L., Veeder, G.J., Gradie, J.C., Lebofsky, L.A., 1989. Three-parameter asteroid taxonomy classifications.. Asteroids II $1151-1161$.

Torppa, J., Kaasalainen, M., Michalowski, T., Kwiatkowski, T., Kryszczyńska, A., Denchev, P., Kowalski, R., 2003. Shapes and rotational properties of thirty asteroids from photometric data. Icarus 164, 346-383.

Usui, F., Kuroda, D., Müller, T.G. Hasegawa, S., Ishiguro, M., Ootsubo, T. Ishihara, D. Kataza, H., Takita, S., Oyabu, S., Ueno, M., Matsuhara, H., Onaka, T., 2011. Asteroid catalog using akari: AKARI/IRC mid-Infrared asteroid survey. Publ. Astron. Soc. Jpn. 63, 1117-1138.

Vachier, F., Berthier, J., Marchis, F., 2012. Determination of binary asteroid orbits with a genetic-based algorithm. Astron. Astrophys. 543, A68. doi:10.1051/ 0004-6361/201118408.

Vernazza, P., Binzel, R.P., Thomas, C.A., DeMeo, F.E., Bus, S.J., Rivkin, A.S., Tokunaga, A.T., 2008. Compositional differences between meteorites and near-Earth asteroids. Nature 454, 858-860. doi:10.1038/nature07154.

Vernazza, P., Carry, B., Emery, J.P., Hora, J.L., Cruikshank, D.P., Binzel, R.P., Jackson, J., Helbert, J., Maturilli, A., 2010. Mid-infrared spectral variability for compositionally similar asteroids: implications for asteroid particle size distributions. Icarus 207, 800-809. doi:10.1016/j.icarus.2010.01.011
Vernazza, P., Marsset, M., Beck, P., Binzel, R.P., Birlan, M., Brunetto, R., Demeo, F.E., Djouadi, Z., Dumas, C., Merouane, S., Mousis, O., Zanda, B., 2015. Interplanetary dust particles as samples of icy asteroids. Astrophys. J. 806, 204. doi:10.1088 0004-637X/806/2/204

Vernazza, P., Zanda, B., Binzel, R.P., Hiroi, T., DeMeo, F.E., Birlan, M., Hewins, R., Ricci, L., Barge, P., Lockhart, M., 2014. Multiple and fast: the accretion of ordinary chondrite parent bodies. Astrophys. J. 791, 120. doi:10.1088/0004-637X/ $791 / 2 / 120$.

Vigan, A., Gry, C., Salter, G., Mesa, D., Homeier, D., Moutou, C., Allard, F., 2015. Highcontrast imaging of sirius a with vlt/sphere: looking for giant planets down to one astronomical unit. Mon. Not. R. Astron. Soc. 454, 129-143. doi:10.1093/ mnras/stv1928.

Vigan, A., Moutou, C., Langlois, M., Allard, F., Boccaletti, A., Carbillet, M., Mouillet, D. Smith, I., 2010. Photometric characterization of exoplanets using angular and spectral differential imaging. Mon. Not. R. Astron. Soc. 407, 71-82. doi:10.1111/j. 1365-2966.2010.16916.x.

Viikinkoski, M., Kaasalainen, M., Ďurech, J., Carry, B., Marsset, M., Fusco, T., Dumas, C., Merline, W.J., Yang, B., Berthier, J., Kervella, P., Vernazza, P., 2015. VLT/SPHERE- And ALMA-based shape reconstruction of asteroid (3) juno. Astron. Astrophys. 581, L3. doi:10.1051/0004-6361/201526626.

Viswanathan, V., Fienga, A., Gastineau, M., Laskar, J., 2017. INPOP17a Planetary ephemerides. Notes Scientifiques et Techniques de l'Institut de mécanique céleste, (ISSN 1621-3823), \#108, ISBN 2-910015-79-3 , 2017, 39 pp. 108.

Weidenschilling, S.J., Chapman, C.R., Davis, D.R., Greenberg, R., Levy, D.H., 1990. Photometric geodesy of main-belt asteroids. III - additional lightcurves. Icarus 86, 402-447. doi:10.1016/0019-1035(90)90227-Z.

Weidenschilling, S.J., Chapman, C.R., Davis, D.R., Greenberg, R., Levy, D.H., Vail, S., 1987. Photometric geodesy of main-belt asteroids. I - lightcurves of 26 large, rapid rotators. Icarus 70, 191-245. doi:10.1016/0019-1035(87)90131-X.

Witasse, O., Lebreton, J.-P., Bird, M.K., Dutta-Roy, R., Folkner, W.M., Preston, R.A., Asmar, S.W., Gurvits, L.I., Pogrebenko, S.V., Avruch, I.M., Campbell, R.M., Bignall, H.E., Garrett, M.A., van Langevelde, H.J., Parsley, S.M., Reynolds, C., Szomoru, A., Reynolds, J.E., Phillips, C.J., Sault, R.J., Tzioumis, A.K., Ghigo, F., Langston, G., Brisken, W., Romney, J.D., Mujunen, A., Ritakari, J., Tingay, S.J., Dodson, R.G., van't Klooster, C.G.M., Blancquaert, T., Coustenis, A., Gendron, E., Sicardy, B., Hirtzig, M., Luz, D., Negrao, A., Kostiuk, T., Livengood, T.A., Hartung, M., de Pater, I., Ádámkovics, M., Lorenz, R.D., Roe, H.G., Schaller, E.L., Brown, M.E., Bouchez, A.H., Trujillo, C.A., Buratti, B.J., Caillault, L., Magin, T., Bourdon, A., Laux, C., 2006. Overview of the coordinated ground-based observations of titan during the huygens mission. J. Geophys. Res. (Planets) 111, 7-19. doi:10.1029/2005JE002640.

Wizinowich, P.L., Acton, D.S., Lai, O., Gathright, J., Lupton, W., Stomski Jr., P.J., 2000. Performance of the W.M. Keck Observatory Natural Guide Star Adaptive Optic Facility: the first year at the telescope, 4007. SPIE, pp. 2-13. doi:10.1117/12. 390368

Yang, B., Wahhaj, Z., Beauvalet, L., Marchis, F., Dumas, C., Marsset, M., Nielsen, E.L., Vachier, F., 2016. Extreme ao observations of two triple asteroid systems with sphere. Astrophys. J. Lett. 820, L35. doi:10.3847/2041-8205/820/2/L35.

Zielenbach, W., 2011. Mass determination studies of 104 large asteroids. Astron. J. 142, 120-128. doi:10.1088/0004-6256/142/4/120.

Zurlo, A., Vigan, A., Mesa, D., Gratton, R., Moutou, C., Langlois, M., Claudi, R.U. Pueyo, L., Boccaletti, A, Baruffolo, A, Beuzit, J-L, Costille, A, Desidera, S. Dohlen, K., Feldt, M., Fusco, T., Henning, T., Kasper, M., Martinez, P., MoellerNilsson, O., Mouillet, D., Pavlov, A., Puget, P., Sauvage, J.-F., Turatto, M., Udry, S., Vakili, F., Waters, R., Wildi, R.F., 2014. Performance of the VLT planet finder SPHERE. i. photometry and astrometry precision with IRDIS and IFS in laboratory. Astron. Astrophys. 572, A85. doi:10.1051/0004-6361/201424204. 